\title{
Existence of solutions of quasilinear elliptic equations on manifolds with conic points
}

\author{
Dissertation \\ zur Erlangung des mathematisch-naturwissenschaftlichen Doktorgrades \\ "Doctor rerum naturalium" \\ der Georg-August-Universität Göttingen
}

vorgelegt von

\section{Thi Thu Huong Nguyen}

aus Hai Phong, Viet Nam

Göttingen 2014 
Referent: Prof. Dr. Ingo Witt

Korreferentin: Prof. Dr. Dorothea Bahns

Ggf. weitere Referentin:

Prof. Dr. Gert Lube (Institut für Numerische und Angewandte Mathematik)

Prof. Dr. Chenchang Zhu (Mathematisches Institut)

Prof. Dr. Folkert Müller-Hoissen (Max-Planck-Institut für Dynamik und Selbstorganisation)

Prof. Dr. Preda Mihailescu (Mathematisches Institut)

Tag der mündliche Prüfung: 


\section{Abstract}

Existence and regularity of solutions of quasilinear elliptic equations in nonsmooth domains have been interesting topics in the development of partial differential equations. The existence of finite-energy solutions of higher-order equations, also those with degenerations and singularities, can be shown by theory of monotone operators and topological methods. There are few results about singular solutions of second-order equations involving the $p$-Laplacian with the Dirac distribution on the right-hand side. So far the existence of singular solutions of higher-order equations with a prescribed asymptotic behavior has not been investigated.

The aims of my dissertation are to look for finite-energy and singular solutions of quasilinear equations on manifolds with conic points. We single out realizations of the $p$-Laplacian in particular, $p \geq 2$, and a conedegenerate operator in general, which belong to the class $(S)_{+}$. Assuming further coercivity conditions and employing mapping degree theory for operators belonging to the class $(S)_{+}$, we obtain existence for the prototypical example of the $p$-Laplacian and for general higher-order equations. 


\section{Zusammenfassung}

Existenz und Regularität der Lösungen der quasilinearen elliptischen Gleichungen in nichtglatten Gebieten sind interessante Themen in der Theorie der partiellen Differentialgleichungen. Die Existenz der Lösungen mit endlichen Energien der Gleichungen höherer Ordnung, sowie Gleichungen mit Degenerationen und Singularitäten, kann durch die Theorie monotoner Operatoren und topologische Methoden gezeigt werden. Existenz singulärer Lösungen der Gleichungen zweiter Ordnung ist nur in dem Fall des p-Laplace Operators mit Dirac'sche-Distribution als rechten Seite bekannt. Bisher ist die Existenz singulärer Lösungen der Gleichungen höherer Ordnung noch unbekannt.

Die Ziele meiner Dissertation sind die Untersuchungen der Existenz der Lösungen mit endlichen und unendlichen Energien der Gleichungen auf Mannigfaltigkeiten mit konischen Punkten. Wir betrachten Erweiterungen des $p$-Laplace Operators, $p \geq 2$, und eines konischen ausgearteten Operators, damit entsprechende Operatoren zu der Klasse $(S)_{+}$ angehören. Seien weiteren Koerzitivität Bedingungen gegeben und verwendet den Abbildungsgrad der Operatoren der Klasse $(S)_{+}$, erhalten wir Existenz für $p$-Laplace Operator beteiligende Gleichungen und für allgemeine Gleichungen höherer Ordnung. 


\section{Acknowledgments}

This dissertation is the result of my working time at the University of Göttingen from October 2009 to October 2013. I would like to express my great thanks to my supervisors, colleagues and family for their caring, guidance and sharing.

Firstly, I am deeply indebted to my advisor Prof. Dr. Ingo Witt, who led me into this interesting topic and always worked with me closely. I am very thankful for his constant help, enthusiastic guidance, patience, and encouragement. Furthermore, I would like to thank Prof. Dr. Dorothea Bahns for her support, understanding and kindness during my study.

Secondly, I would like to express my thanks to all members of the graduate seminar "Analysis of partial differential equations" at the Mathematical Institute not only for many helpful discussions about my topics, but also for interesting introduction to theirs, which broaden my knowledge in PDE a lot. My special thanks go to Jiguang and Sutanu for many encouraging and motivating talks whenever I was depressed. I am also thankful for the scientific support of the Research Training Group 1493 "Mathematical structures in modern quantum physics".

Finally, my deepest gratitude goes to my big family, especially my parents for their love, encouragement and help even from far apart, and my husband for his support and encouragement not only in life but also at work. They were constantly by my side during my study. 



\section{Contents}

Abstract i

Acknowledgments iii

\begin{tabular}{lll}
\hline 1 & Introduction & 1
\end{tabular}

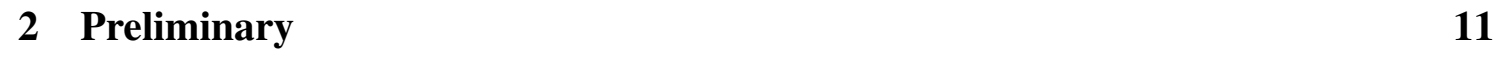

2.1 Analysis on manifolds with conic points . . . . . . . . . . . . 11

2.1 .1 Manifolds with conic points . . . . . . . . . . . . 11

2.1 .2 Cone-degenerate differential operators . . . . . . . . . . . 12

2.1 .3 Function spaces . . . . . . . . . . . . . . . 15

2.1.3.1 Weighted Sobolev spaces $\mathcal{H}_{p}^{s, \gamma}(X ; E) \ldots \ldots . . . .15$

2.1.3.2 Embedding theorems . . . . . . . . . . 17

2.1 .4 Mapping properties of cone-degenerate differential operators . . . 19

2.2 Existence theorems for monotone operators . . . . . . . . . . 20

2.3 Mapping degree of generalized monotone mappings . . . . . . . . . 23

2.3 .1 Generalized monotone mappings . . . . . . . . . . . . 23

2.3 .2 Degree of generalized monotone mappings . . . . . . . . . . 24

2.3 .3 Properties of the mapping degree . . . . . . . . . . . . 25

2.3 .4 Existence theorems . . . . . . . . . . . . . . . 26

2.4 Inequalities for vectors $\ldots \ldots \ldots \ldots$

\begin{tabular}{|ll|}
\hline 3 & Second-order equations \\
\hline
\end{tabular}

3.1 Existence of finite energy solutions $\ldots \ldots \ldots 33$

3.2 Singular solutions to equations involving $p$-Laplacian . . . . . . . . . 41

3.2.1 Singular solutions of cone $p$-Laplacian . . . . . . . . . . . 43

3.2.2 Singular solutions of the $p$-Laplacian with an absorption term . . 48 
3.3 Second-order equations $\ldots \ldots \ldots \ldots$. . . . . . . . . . . 51

3.3 .1 Serrin's case . . . . . . . . . . . . . . . . 56

3.3 .2 Equations with an absorption term $g(u) \ldots \ldots$. . . . . . 57

3.3 .3 General assumption about coercivity . . . . . . . . . . . . . 57

4 Higher-order equations

$4.1 \quad$ Cone algebra with asymptotics $C^{v}(X, \mathfrak{g}) \ldots \ldots \ldots$. . . . . . . 60

4.1 .1 Mellin transformation . . . . . . . . . . . . . 61

4.1 .2 Cone weighted Sobolev spaces with asymptotics . . . . . . . 62

4.1 .3 Green operators . . . . . . . . . . . . . . . . 65

4.1 .4 Mellin pseudo-differential operators . . . . . . . . . . . 67

4.1.5 Ellipticity and regularity with asymptotics . . . . . . . . . . 70

4.2 Extensions of cone-degenerate differential operators . . . . . . . . . . 72

4.3 Finite energy solutions . . . . . . . . . . . . . . . . 75

4.4 Existence of singular solutions $\ldots \ldots \ldots \ldots$. . . . . . . . . 81

\begin{tabular}{lll}
\hline 5 & Conclusion & 87
\end{tabular}

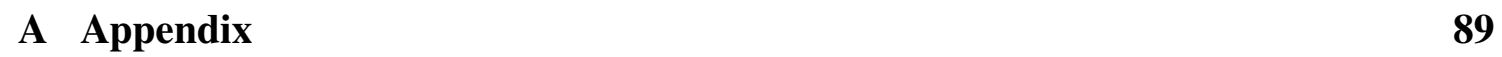

A.1 Degree of mappings of class $\alpha \ldots \ldots \ldots$. . . . . . . . . . . . 89

A.2 Closed extensions of symmetric operators . . . . . . . . . . . . 93

\begin{tabular}{ll}
\hline Bibliography & 97
\end{tabular} 


\section{Introduction}

The present thesis is devoted to the study of the existence of finite-energy and singular solutions of quasilinear elliptic equations on manifolds with conic points. We will use alternative functional analysis methods to prove first for second-order equations, in particular for the prototypical example of the $p$-Laplacian, and then for higher-order equations.

As mentioned, our consideration takes place on manifolds with conic points. After blow-up procedure, or equivalently, introducing polar coordinates near those points, one actually works on a smooth manifold $X$ whose cross-section $Y$ is a smooth manifold without boundary. The geometry of the original object is captured by the type of degeneration of observed operators. In the present case of a manifold with conic points, the corresponding differential operators are called cone-degenerate differential operators, which are given in local coordinates in a collar neighborhood $(0,1] \times Y$ of the boundary by

$$
A=t^{-v} \sum_{k=0}^{v} a_{k}(t)\left(-t \frac{\partial}{\partial t}\right)^{k},
$$

where the coefficients $a_{k}(t) \in C^{\infty}\left([0,1), \operatorname{Diff}^{\nu-k}(Y)\right)$. The cone degeneracy shows up in the derivative $\left(-t \partial_{t}\right)$. The analysis on manifolds with conic points, and in more general stratified spaces, has been established by Schulze [11, 33]. The resulting cone calculus will play an important role in this work.

We are particularly interested in quasilinear equations of divergence form, that are equations of the form

$$
A^{\prime} \mathcal{F}(x, A u)+\mathcal{G}(x, B u)=f \quad \text { in } X \backslash Y,
$$

where the operators $A \in \operatorname{Diff}_{c}^{v}(X ; E, F), B \in \operatorname{Diff}_{c}^{\gamma^{\prime}}(X ; E, G),\left(v, v^{\prime} \geq 1, v^{\prime}<2 v\right)$, are cone-degenerate differential operators with real coefficients acting between sections of real vector bundles $E, F$ and $E, G$ over $X \backslash Y$, respectively. The nonlinearities

$$
\mathcal{F}: F \rightarrow F^{\prime}, \quad \mathcal{G}: G \rightarrow E^{\prime}
$$


are fiber-preserving maps. For $x \in X \backslash Y$, we write $\mathcal{F}(x, \cdot)$ and $\mathcal{G}(x, \cdot)$ in place of the induced fibrewise maps $\mathcal{F}_{x}: F_{x} \rightarrow F_{x}^{\prime}$ and $\mathcal{G}_{x}: E_{x} \rightarrow G_{x}$, respectively. Here $E^{\prime}$ and $F^{\prime}$ are the dual bundles of $E$ and $F$, respectively. We furnish $X$ with a measure $d \mu$ which is $t^{N-p \gamma} d t d y$ close to $Y, d y$ is a measure on $Y, \gamma \in \mathbb{R}$ is a fix weight, $\gamma<\frac{N+1}{p}, \operatorname{dim} X=N+1$. Then $A^{\prime} \in \operatorname{Diff}_{c}^{v}\left(X ; F^{\prime}, E^{\prime}\right)$ denotes the adjoint operator of $A$ with respect to the scalar product in $L^{2}(X, d \mu)$.

A prototypical example of quasilinear equations is equation involving the $p$-Laplace operator, which in our setting is written as

$$
\Delta_{p} u=-\operatorname{div}_{g}\left(\left|\nabla_{g} u\right|^{p-2}\left|\nabla_{g} u\right|\right)
$$

where $p>1, \operatorname{div}_{g} \in \operatorname{Diff}_{c}^{1}\left(X,{ }^{c} T X, \mathbb{R}\right), \nabla_{g} \in \operatorname{Diff}_{c}^{1}\left(X, \mathbb{R}^{c}{ }^{c} T X\right)$ are the divergence and the gradient operators with respect to a cone metric $g, g=d t^{2}+t^{2} g_{Y}$, when a Riemannian metric $g_{Y}$ on $Y$ is given. These operators act between the trivial bundle and the cone tangent bundle on $X$ (the latter bundle will be defined in (2.2)). In the following, we will drop the reference to the metric, that is we write $\nabla$ and div.

Existence and regularity of quasilinear elliptic equations have been of great interest since the fifties of the last century. The first systematic investigation was the monograph of Ladyzhenskaia and Ural'tzeva, in which the authors considered second-order equations in regular domains in $\mathbb{R}^{n}$. Their approach using Leray-Schauder fixed point theorem, also presented in the book by Gilbarg and Trudinger ([14] $)$, relies much on interior and boundary estimates. Another approach to second-order equations is the method of sub- and supersolutions, many fundamental results like Harnack inequality, comparison principle ... are developed further for second-order equations with degeneration, we refer to the book by Heinonen, Kilpeläinen and Martio ([17]) on nonlinear potential theory.

Since the nineteen sixties, there has been a broad development in the study of higherorder quasilinear elliptic equations. The work of Vishik used a modification of the Galerkin approximation method. Subsequent progress were connected to the theory of monotone and more general operators in the famous work of Browder [4], Leray-Lions [24] ... Quasilinear elliptic equations of divergence form fit well in this framework, especially when they satisfy the so-called coercivity property. We will utilize existence theorems by those authors in chapter 3. Solvability of equations involving noncoercive operators was also investigated, for example odd operators in the work of Pokhozhaev, Browder, Petryshyn, Skrypnik, see [38, Chapter 1] for more details. These results can also be obtained by using the so-called mapping degree theory for generalized monotone mappings, 
which was developed independently by Browder and Skrypnik at the end of the sixties and the beginning of the seventies. This mapping degree, constructed for the class of mappings possessing property $\alpha$ (or termed $(S)_{+}$by Browder), generalizes the mapping degree of finite-dimensional operators in the sense that it inherits all the properties of the Brouwer degree. One has a simple criterion for the existence of solutions of equations including such operators, namely when this degree is nonzero the equation is solvable, see theorem 2.30. In particular, this criterion holds for coercive or odd operators. We refer to the book by Drábek-Milota [10] for a nice introduction to mapping degree theory and to the monograph by Skrypnik [38] for various general framework for solving operator equations and fruitful examples of quasilinear and fully nonlinear equations in regular domains. However, compared to the use of other methods, for example monotone theory, this method works for nonlinearities having a slightly smaller growth. These functional analysis methods applied to equations with degeneration and singularity were presented in the book by Drábek, Kufner and Nicolosi, see [9].

In this work, we will utilize these functional methods to establish existence of solutions to second- and higher-order equations on manifolds with conic points. The proofs go smoothly at least in the case of finite-energy solutions, with an adaptation to the nonsmooth situation. We take into account the analysis on manifolds with singularities presented in the work of Egorov-Schulze ([11]), Schulze ([33]) in $L^{2}$ case, and SeilerSchrohe ([31]) in $L^{p}$ case. We obtain in chapter 3 section 3.1 and chapter 4 , section 4.3 the existence of finite-energy solutions of quasilinear equations of second- and higherorders, respectively, namely

Theorem 1.1 (Theorem 3.3). Consider the equation

$$
-\operatorname{div} \mathcal{F}(x, \nabla u)-\operatorname{div} g_{1}(x, u)+g_{2}(x, u, \nabla u)=f(x), x \in X \backslash Y .
$$

Suppose that the nonlinearities subject to the conditions (3.2), (3.3), (3.4), (3.5), and furthermore, for almost all $(x, u, q) \in X \times \mathbb{R} \times{ }^{c} T X$, it holds

$$
\mathcal{F}(x, q) q+g_{1}(x, u) q+g_{2}(x, u, q) u \geq C_{2}|q|^{p}+C_{3}|u|^{p}-C_{4} .
$$

Given $f \in \mathcal{H}_{p^{\prime}}^{-1,-1}\left(X ; E^{\prime}\right)$, then the equation (1.3) has at least one solution $u \in \mathcal{H}_{p}^{1,1}(X ; E)$.

Let us emphasize that the mapping degree approach depends much on the ability of proving that the induced operator satisfies the property $\alpha(D)$ for some subset $D$ of the 
function space, which depends on the structure of the nonlinearities appearing in the original equation. The $p$-Laplace operator as the prototype for quasilinear elliptic equations, however, does not satisfy (1.4). Therefore, we also present related results at the end of section 3.1, obtained by modifying the structural conditions for the nonlinearities and verifying the corresponding function spaces in which we look for solutions.

Theorem 1.2 (Theorem 3.5). Suppose that the cone Laplacian $\Delta_{g}$ is cone-elliptic with respect to the weight line $\mathfrak{R}_{z}=1$ and the nonlinearities subject to the conditions (3.2), (3.3), (3.4), (3.5). Furthermore, assume that for almost all $(x, u, q) \in X \times \mathbb{R} \times{ }^{c} T X$, it holds

$$
\mathcal{F}(x, q) q+g_{1}(x, u) q+g_{2}(x, u, q) u \geq C_{2}|q|^{p}-C_{4} .
$$

Given $f \in \mathcal{H}_{p^{\prime}}^{-1,-1}(X)$ which is perpendicular to $\operatorname{Ker} \nabla$, there exists a solution $u \in \mathcal{H}_{p}^{1,1}(X)$ of 1.3 .

The corresponding result in the case of higher-order equations is

Theorem 1.3 (Theorem 4.33). Consider the equation

$$
A^{\prime} \mathcal{F}(x, A u)+\mathcal{G}(x, B u)=f, x \in X \backslash Y .
$$

Assume that the nonlinearities $\mathcal{F}: X \times E \rightarrow F$ and $\mathcal{G}: X \times G \rightarrow E$ are continuous and subject to the following growth conditions for almost $x \in X \backslash Y, q \in E_{x}, z \in G_{x}$.

(F1) $\|\mathcal{F}(x, q)\|_{F} \leq h_{1}(x)+|q|^{p-1}$, where $h_{1}(x) \in \mathcal{H}_{p^{\prime}}^{0,0}(X)$,

(F2) $h_{F}(\mathcal{F}(x, q), q) \geq C_{0}\|q\|_{F}^{p}-C_{1}$,

(F3) $h_{F}\left(\mathcal{F}\left(x, q_{1}\right)-\mathcal{F}\left(x, q_{2}\right), q_{1}-q_{2}\right)>0$ as $q_{1}, q_{2} \in F_{x}, q_{1} \neq q_{2}$,

(G1) $h_{E}(G(x, z), z) \leq h_{2}(x)+\|z\|_{G}^{p-1-\varepsilon}$, where $h_{2}(x) \in \mathcal{H}_{p^{\prime}}^{0,0}(X), \varepsilon \geq 0$,

(C) $h_{F}(\mathcal{F}(x, q), q)+h_{E}(\mathcal{G}(x, \xi), u) \geq C_{0}\|q\|_{F}^{p}+C_{2}\|u\|_{E}^{p}$,

where $h_{E}, h_{F}$ are bundle metrics on $E$ and $F$, respectively.

Consider a domain D such that

$$
D\left(A_{\min }\right) \subseteq D \subseteq D\left(A_{\max }\right)
$$

Suppose further that either $B: D \rightarrow \mathcal{H}_{p}^{0,0}(X ; G)$ is compact or it is continuous and $\varepsilon>0$. Then for all $f \in D^{\prime}$, there exists at least one solution to the equation (1.6). 
The next goal of this thesis is to search for singular solutions. Going beyond finiteenergy solutions, there are several sorts of singular solutions, for instance renormalized solutions $([[2,7])$, viscosity solutions $([[18])$, in which classes the authors obtained the existence and (partial) uniqueness. The singular solutions we are interested in have infinite energy in the natural domain of definition of the operator. The first understanding of the behavior of such solutions was presented in the seminal papers by Serrin [35, 36]. The author investigated second-order equations in the $\mathbb{R}^{N+1}$ setting which are of the type

$$
\sum_{i=1}^{N+1} \frac{\partial}{\partial x_{i}} \mathcal{A}_{i}(x, u, \nabla u)=\mathcal{A}(x, u, \nabla u), x \in \Omega \subset \mathbb{R}^{N+1},
$$

and in the case the nonlinear growth on the left-hand side dominates the one on the righthand side, namely the following structural conditions

$$
\begin{gathered}
\left|\mathcal{A}_{i}(x, u, z)\right| \leq a|z|^{p-1}+b|u|^{p-1}+e, \\
|\mathcal{A}(x, u, z)| \leq c|z|^{p-1}+d|u|^{p-1}+f, \\
z \cdot \mathcal{A}(x, u, z) \geq a^{-1}|z|^{p}-d|u|^{p}-g,
\end{gathered}
$$

where $p>1$ is a fixed constant, $a$ is a positive constant, and the coefficients $b$ through $g$ are measurable functions of $x$ belonging to certain Lebesgue spaces. In the case $p>N+1$ and the solution $u$ is bounded from below or above, then the singularity is removable, $u$ can be extended to be a continuous solution in the whole domain $\Omega$. Moreover, for such $p$, one can prove existence result by using variational methods. We are not interested in this case. In the case $1<p \leq N+1$, and $u$ is bounded from below but not from above, then it behaves like the singular $p$-harmonic function $\mu_{p}(x)$ near the singularity. We recall these results in the following theorem.

Theorem 1.4 ([35, Theorem 12]). Let u be a continuous solution of the equation (1.7) in $\Omega \backslash\{0\}$. Suppose that $u$ is bounded from below, that is $u \geq L$ for some constant $L$. Then either $u$ has a removable singularity at 0 , or else

$$
u(x) \sim \mu_{p}(x)=\left\{\begin{array}{ll}
|x|^{-\frac{N+1-p}{p-1}} & \text { if } p<N+1, \\
-\log |x| & \text { if } p=N+1,
\end{array} \quad \text { as }|x| \rightarrow 0,\right.
$$

up to a positive multiplicative constant.

Concerning existence, Serrin considered in [35] an ideal case in which the problem could be solved by approximation. 
In the reverse case, when the nonlinear growth the right-hand side dominates the one on the left-hand side, there have been several important existence results about equations involving the $p$-Laplace operator and the capillarity equation which were presented in the book by Véron ([41]). Véron and his collaborators found out the asymptotic behavior of singular solutions of the two following equations

$$
-\operatorname{div}\left(|\nabla u|^{p-2} \nabla u\right)+g(u)=0
$$

and

$$
-\operatorname{div}\left(|\nabla u|^{p-2} \nabla u\right)=u^{q}, u>0,
$$

where $p-1<q<\frac{(N+1)(p-1)}{N+1-p}$ when $1<p<N+1$, and $p-1<q$ when $p=N+1$, and $g(u)$ subjects to further conditions which corresponds to an absorption term. In the case of an absorption term (1.9) and in the sub-critical case of a source term (1.10), the authors showed that singular solutions behave again like the singular $p$-harmonic function $u(x) \sim \mu_{p}(x)$ as $|x| \rightarrow 0$.

Knowledge of the behavior of solutions in this observation is important when it is used in the approximation scheme and by comparison principle, the approximate solutions are shown to be uniformly bounded in the space $C^{1, \alpha}(\Omega \backslash\{0\})$ and consequently converge. We recall two existence theorems: one by Friedman-Véron [12] for equations (1.9] and the other by Kichenassamy-Véron [21] for the $p$-Laplacian

$$
-\operatorname{div}\left(|\nabla u|^{p-2} \nabla u\right)=\delta \text { in } \mathcal{D}^{\prime}(\Omega)
$$

Concerning the capillarity equation, Véron summarized in [41, Section 5.4] the results of Concus and Finn, the problem is to find singular solutions of the equation

$$
\operatorname{div}\left(\frac{\nabla u}{\sqrt{1+|\nabla u|^{2}}}\right)=K v, K<0 .
$$

With the prescribed asymptotic $u(r) \sim-\frac{1}{r}$ and the search for solutions of the form

$$
u(r)=-\frac{1}{r}+\frac{N+4}{2 N} r^{3}+r^{3} v(r)
$$

these authors concluded the existence for the capillarity equation. A simplified proof of existence of singular solution was considered by Bidaut-Véron, where the author looked for the function $z(r)$, where

$$
z(r)=\frac{u_{r}(r)}{\sqrt{1+u_{r}^{2}(r)}},
$$


in the form

$$
z(r)=1-\frac{r^{4}}{2}+r^{6} w(r)
$$

At the end, these solutions were shown to coincide, although in Concus and Finn's approach the corresponding $z(r)$ is given in the form

$$
z(r)=1-\frac{r^{4}}{2}+r^{8} w(r)
$$

It means that the singular solution is uniquely determined by its singular behavior. Let us also emphasize that so far there has been no results for singular solutions of higher-order quasilinear elliptic equations.

We have noticed that the knowledge of the leading behavior of solutions is essential. We assume the existence of a formal solution of the observed equation. Its leading behavior is found by calculating formally and balancing the leading terms on both sides of the equation. Let us emphasize that, in case of semilinear equations, one can always subtract the singular part from the solution and, after finite times of transformation of the equation, actually looks for a finite-energy one. In the case of a quasilinear equation, we cannot reduce to the situation of a finite-energy solution with the same principal part and even in the procedure of finding the leading order term, one needs to choose the realization of the operator in use. We make another assumption, namely there are no further resonances, it means the terms with prescribed coefficients. In other words, only the leading term must be prescribed, otherwise we have freedom to choose how smooth the remainder is by choosing an appropriate function space containing it. The existence of the remainder will result the existence of the singular solution.

We start with equations involving cone $p$-Laplace operator as a model case and obtain the following results:

Theorem 1.5 (Theorem 3.10). Let $p \geq 2$. Consider the equation

$$
-\operatorname{div}\left(|\nabla u|^{p-2} \nabla u\right)=f(x), x \in X \backslash Y .
$$

Define $\mathfrak{X}:=\mathcal{H}^{1, \gamma}(X) \cap \mathcal{H}_{p}^{1,1}(X)$, where $\gamma=\frac{N(p-2)}{2(p-1)}+1$. Then, given an $f \in \mathfrak{B}^{*}$, where $\mathfrak{B}$ is a complement of $\operatorname{Ker} \nabla$ in $\mathfrak{X}$, there exists a unique solution modulo $\operatorname{Ker} \nabla$ to (1.12) which is of the form $e+v$, where $e \sim C t^{-\alpha}$ is the approximate solution such that $\Delta_{p} e \in \mathfrak{B}^{*}$, where $\alpha=\frac{N+1-p}{p-1}$ is the Serrin's exponent, and $v \in \mathfrak{X}$.

Theorem 1.6 (Theorem 3.11). Let $p \geq 2$. Consider the equation

$$
-\operatorname{div}\left(|\nabla u|^{p-2} \nabla u\right)+g(u)=f(x), x \in X \backslash Y .
$$


Assume that the nonlinearity $g$ is an increasing function satisfying

$$
0 \leq g^{\prime}(s) \leq C|s|^{p-2}, \text { for all } s \in \mathbb{R}
$$

Given an $f \in \mathfrak{B}^{*}$, there exists at least one solution to 1.13 which is of the form $e+v$, where $e \sim C t^{-\alpha}$ such that $\Delta_{p} e \in \mathfrak{B}^{*}$ and $v \in \mathfrak{X}$.

These theorems will be proved by using Browder and Leray-Lions theorems, respectively. The common assumption in these theorems is the coercivity of the operator. We will extend the range of our applicable equations by using mapping degree of generalized monotone operators. We extend the result of Skrypnik about the $\alpha$ property of the $p$ Laplacian which is considered in more general function spaces. Here the specific form of the operator plays a vital role, and we use this model to obtain further results as follows.

Theorem 1.7 (Theorem 3.16). Let $p \geq 2$. Assume that the nonlinearities $\mathcal{F}, \mathcal{G}$ satisfy growth conditions (3.22), (3.23), (3.24), (3.25), and for $\left(x, u_{1}, q_{1}\right),\left(x, u_{2}, q_{2}\right) \in X \times \mathbb{R} \times{ }^{c} T X$, it holds

$$
\begin{aligned}
& \left\langle\mathcal{F}\left(x, q_{1}\right)-\mathcal{F}\left(x, q_{2}\right), q_{1}-q_{2}\right\rangle+\left\langle g_{1}\left(x, u_{1}\right)-g_{1}\left(x, u_{2}\right), q_{1}-q_{2}\right\rangle \\
& \quad+\left\langle g_{2}\left(x, u_{1}, q_{1}\right)-g_{2}\left(x, u_{2}, q_{2}\right), u_{1}-u_{2}\right\rangle \geq\left(\left|q_{1}\right|^{p-2}+\left|q_{2}\right|^{p-2}\right)\left|q_{1}-q_{2}\right|^{2} .
\end{aligned}
$$

Then for all $f \in \mathfrak{B}^{*}$, the equation

$$
-\operatorname{div} \mathcal{F}(x, \nabla u)-\operatorname{div} g_{1}(x, u)+g_{2}(x, u, \nabla u)=f(x), x \in X \backslash Y,
$$

has at least one solution in $\mathfrak{X}$.

This result is generalized to higher-order equations in chapter 4 .

Theorem 1.8 (Theorem 4.37). Let $p>2$. Suppose that $A^{\prime} A$ is an elliptic operator with respect to $\gamma, \gamma+v$, and at least one of the mappings $B: \mathfrak{W} \rightarrow \mathcal{H}_{p}^{0, \gamma}\left(X ; G_{0}\right) \cap \mathcal{H}^{0, \rho}\left(X ; G_{0}\right)$ and $C: \mathfrak{B} \rightarrow \mathcal{H}_{p}^{0, \gamma}\left(X ; G_{1}\right) \cap \mathcal{H}^{0, \rho}\left(X ; G_{1}\right)$ is a compact operator. Suppose further that the nonlinearity $\mathcal{F}$ satisfies the growth conditions (4.23, 4.24) and $\mathcal{G}: X \times G_{0} \rightarrow G_{1}^{\prime}$ satisfies

$$
\left\|\mathcal{G}\left(x, z_{1}\right)-\mathcal{G}\left(x, z_{2}\right)\right\|_{G_{1}^{\prime}} \leq C\left\|z_{1}-z_{2}\right\|_{G_{0}}\left(\left\|z_{1}\right\|_{G_{0}}^{p-2-\varepsilon}+\left\|z_{2}\right\|_{G_{0}}^{p-2-\varepsilon}\right)
$$

for all $\left(x, z_{i}\right) \in G_{0}, i=1,2$, here $0<\varepsilon<p-2$.

Furthermore, let one of the following conditions hold for $\left(x, q_{1}\right),\left(x, q_{2}\right) \in F$ and $\left(x, z_{1}\right),\left(x, z_{2}\right) \in G_{0}$ : 
a) If $B=C$ and $\mathcal{G}$ is a nondecreasing function.

b) $\left\|\mathcal{G}\left(x, z_{1}\right)-\mathcal{G}\left(x, z_{2}\right)\right\|_{G_{1}^{\prime}} \leq C\left\|z_{1}-z_{2}\right\|_{G_{0}}^{\sigma}\left(\left\|z_{1}\right\|_{G_{0}}^{p-2-\varepsilon}+\left\|z_{2}\right\|_{G_{0}}^{p-2-\varepsilon}\right), 0<\sigma<1$.

c) For $\left(x, \bar{z}_{1}\right),\left(x, \bar{z}_{2}\right) \in G_{1}$, it holds

$$
\left\langle\mathcal{F}\left(x, q_{1}\right)-\mathcal{F}\left(x, q_{2}\right), q_{1}-q_{2}\right\rangle+\left\langle\mathcal{G}\left(x, z_{1}\right)-\mathcal{G}\left(x, z_{2}\right), \bar{z}_{1}-\bar{z}_{2}\right\rangle \geq\left(\left|q_{1}\right|^{p-2}+\left|q_{2}\right|^{p-2}\right)\left|q_{1}-q_{2}\right|^{2}
$$

Then for any $f \in \Xi^{*}$, the equation

$$
A^{\prime} \mathcal{F}(x, A u)+\mathcal{G}(x, B u)=f \quad \text { in } X \backslash Y
$$

has an actual solution $u$ belonging to the affine space $e+\Xi$ provided that it has an approximate solution $e \sim \varphi(y) t^{-\lambda_{0}}$ that $A^{\prime} \mathcal{F}(x, A e)+\mathcal{G}(x, B e) \in \Xi^{*}$.

So far, we have discussed the mathematical interest of solving quasilinear elliptic equations. Nevertheless, quasilinear equations appear in many problems in mathematical physics. For instance, we mentioned the capillarity equation (1.11) and the mean curvature equation ( $(1.11)$ with $K=0$ ). The second-order equation involving the $p$-Laplacian, namely

$$
-\operatorname{div}\left(|\nabla u|^{p-2} \nabla u\right)+\lambda u=0, p>1, \lambda>0
$$

appears in the study of non-Newtonian fluids. Different values of $p$ mean different kinds of fluid media, namely, media with $p>2$ are called dilatant, those with $p>2$ are called pseudoplastics, for $p=2$ the media is a Newtonian fluid. For further examples, we refer to the book by Díaz ([8] $)$.

This thesis is divided into four chapters beginning with the introduction and summary of results. In the second chapter, we present basic, necessary knowledge for the whole work, including the analysis on manifolds with conic points and functional analysis tools for solvability of operator equations. In Section 2.3, we recall the mapping degree theory for generalized monotone mappings constructed by Skrypnik. This chapter ends with some important inequalities for vectors.

The third chapter is about existence of finite-energy and singular solutions to secondorder equations. Results concerning finite-energy solutions are modified on the singular manifolds setting. Results concerning singular solutions start with the consideration of the $p$-Laplacian. All growth conditions on nonlinearities later are based on this prototypical example. We obtain existence in functional sense. 
In chapter 4, we prove existence results for higher-order equations. The investigation of finite energy solutions is quite straightforward. Section 4.2 is devoted to the cone algebra, which provides the basis for working with higher-order equations. The last part of this chapter contains proofs of some inequalities for functions belonging to weighted Sobolev spaces and of the existence of singular solutions.

The thesis ends with an appendix, where we recall in detail the construction of the mapping degree for generalized monotone mappings and descriptions of the minimal and maximal extensions of an elliptic cone-degenerate operator. 


\section{Preliminaries}

In the first chapter, we recall general background for the whole work including the introduction of the observed geometric object and analysis on it: the class of operators, their mapping properties between appropriate cone Sobolev spaces; we collect some existence results in the theory of monotone operators, namely the Browder and Leray-Lions theorems, and the degree of generalized monotone mappings. At the end of this chapter, there are some elementary inequalities for vectors, which will be used to derive properties of the $p$-Laplacian.

\subsection{Analysis on manifolds with conic points}

We begin with describing the geometric objects under consideration together with typical differential operators on them. Adequate class of functional spaces is introduced and the mapping properties of operators acting between these spaces are summarized. Ingredients are taken from the book [11] by Egorov and Schulze in the $L^{2}$ case, and the paper [31] in the $L^{p}$ case.

\subsubsection{Manifolds with conic points}

Definition 2.1. [11, Section 7.1.2] A finite dimensional manifold $B$ with conic singularities is a second countable Hausdorff space with a finite subset

$$
B_{0}=\left\{b_{1}, \ldots, b_{m}\right\} \subset B
$$

of conic points such that the following properties hold:

i) $B \backslash B_{0}$ is a smooth manifold,

ii) every $b \in B_{0}$ has an open neighborhood $V$ in $B$, such that there exists a diffeomorphism $\phi: V \backslash\{b\} \rightarrow Y(b)^{\wedge}$ for some closed compact $C^{\infty}$ manifold $Y(b)$, 
and this diffeomorphism is extendable to a homeomorphism $\bar{\phi}: V \rightarrow Y(b)^{\triangle}$, here $Y(b)^{\wedge}=\mathbb{R}_{+} \times Y(b)$ is the open stretched manifold associated with $Y(b)$, and $Y(b)^{\triangleleft}=$ $\overline{\mathbb{R}}_{+} \times Y(b) /(\{0\} \times Y(b))$,

iii) if $\psi: V \backslash\{b\} \rightarrow Y(b)^{\wedge}$ is another diffeomorphism, which is extendable to $V$, then

$$
\phi \psi^{-1}: Y(b)^{\wedge} \rightarrow Y(b)^{\wedge}
$$

is the restriction of some diffeomorphism $\overline{\mathbb{R}}_{+} \times Y(b) \rightarrow \overline{\mathbb{R}}_{+} \times Y(b)$ to $\mathbb{R}_{+} \times Y(b)$.

By definition, we can associate $B$ with its stretched manifold $X$ whose boundary is a compact smooth manifold $Y \cong \bigcup_{b \in B_{0}} Y(b)$ and whose interior is int $X \cong B \backslash B_{0}$. For simplicity, in this work we consider manifolds with only one conic point. Equivalently, we work with a smooth, compact manifold $X$ of dimension $N+1$ having compact connected boundary $Y$. Near $Y$ we fix a collar neighborhood $U \cong[0,1) \times Y$ and local coordinates $(t, y)$. We extend $t$ smoothly to a defining function on $X$ and the boundary $Y$ is then given by $\{t=0\}$.

Example 2.2. Given a closed compact smooth manifold $X$ of dimension $N+1$ and fix a point $x_{0} \in X$, then $X$ can be considered as a manifold with a conic singularity at $x_{0}$. The cross-section $Y$ in this example is the sphere $\mathbb{S}^{N}$.

Let $Y$ be an arbitrary closed compact smooth manifold, then $B=Y^{\Delta}$ is a manifold with conic singularity and its stretched manifold is $X=Y^{\wedge}$.

\subsubsection{Cone-degenerate differential operators}

Considering a manifold $X$ with boundary $Y$, by a vector bundle $E$ over $X$ we mean that $E$ is a vector bundle over the interior int $X$ of $X$ and $E$ is also the pullback of a vector bundle $E_{Y}$ over the boundary $Y$ under the canonical projection $\Pi: U \cong[0,1) \times Y \rightarrow Y$. For simple notation, we will denote the bundle $E_{Y}$ by $\left.E\right|_{Y}$.

Definition 2.3. Let $E$ and $F$ be two real vector bundles over $X$. A $v$-th order differential operator $A$ with smooth coefficients acting between sections of $E$ and $F$ is called a conedegenerate operator if it has the following form in a neighborhood $U$ of the boundary

$$
\left.A\right|_{U}=t^{-v} \sum_{k=0}^{v} a_{k}(t)\left(-t \frac{\partial}{\partial t}\right)^{k} \text {, }
$$


where the coefficients $a_{k}(t) \in C^{\infty}\left([0,1), \operatorname{Diff}^{\nu-k}\left(Y ;\left.E\right|_{Y},\left.F\right|_{Y}\right)\right)$ are differential operators acting between sections of $\left.E\right|_{Y}$ and $\left.F\right|_{Y}$.

We denote by $\operatorname{Diff}_{c}^{v}(X ; E, F)$ the class of cone-degenerate differential operators of or$\operatorname{der} v$ acting between sections of $E$ and $F$. For simple notation, we will omit to indicate these vector bundles.

Example 2.4. Let $g_{Y}(t)$ be a $t$-dependent family of Riemannian metrics on a closed compact $C^{\infty}$ manifold $Y$ which is infinitely differentiable in $t \in[0,1)$, and $g_{Y}(0)$ depends only on $y$ and $d y$. Then

$$
g:=d t^{2}+t^{2} g_{Y}(t)
$$

is a Riemannian metric on $X$. The gradient and the divergence operators with respect to this metric are first order operators $\operatorname{div}_{g} \in \operatorname{Diff}_{c}^{1}\left(X,{ }^{c} T X, \mathbb{R}\right), \nabla_{g} \in \operatorname{Diff}_{c}^{1}\left(X, \mathbb{R},{ }^{c} T X\right)$, respectively. Here, ${ }^{c} T X$ is the cone tangent bundle which has a basis expressed in local coordinates as follows:

$$
\frac{\partial}{\partial t}, t^{-1} \frac{\partial}{\partial y_{1}}, \ldots, t^{-1} \frac{\partial}{\partial y_{N}}
$$

This basis has a dual basis in the cone cotangent bundle ${ }^{c} T^{*} X$ given by

$$
d t, t d y_{1}, \ldots, t d y_{N}
$$

The $p$-Laplace operator $\operatorname{div}_{g}\left(\left|\nabla_{g} u\right|^{p-2} \nabla_{g} u\right)$ is a cone-degenerate operator of second-order acting between sections of trivial bundles $X \times \mathbb{R}$ and $X \times \mathbb{R}$.

In the interior of $X$, a cone-degenerate differential operator is a differential operator in the usual sense, therefore, it has a standard interior principal symbol $\sigma_{\psi}^{v}(A) \in$ $C^{\infty}\left(T^{*}\right.$ int $\left.X \backslash 0 ; \operatorname{Hom}\left(\pi^{*} E, \pi^{*} F\right)\right)$, where $\pi^{*} E, \pi^{*} F$ are the pullback bundles of $E$ and $F$, respectively, by the projection $\pi: T^{*}$ int $X \backslash 0 \rightarrow$ int $X$ from the cotangent bundle with zero section removed to the first component. Furthermore, we associate with $A$ two other symbols: the compressed principal symbol $\widetilde{\sigma}_{\psi}^{v}(A) \in C^{\infty}\left({ }^{c} T^{*} X \backslash 0\right)$ which is given in local coordinates by

$$
\widetilde{\sigma}_{\psi}^{v}(A)(t, y, \widetilde{\tau}, \eta)=\sum_{k=0}^{v} \sigma_{\psi}^{v-k}\left(a_{k}\right)(t, y, \eta)(-i \widetilde{\tau})^{k},
$$

and the principal conormal (or Mellin) symbol is given by

$$
\sigma_{M}^{v}(A)(z)=\sum_{k=0}^{v} a_{k}(0) z^{k}, z \in \mathbb{C}
$$


The conormal symbol is a polynomial in $z$ which takes values in the class of differential operators on the boundary of order at most $v$. In particular, the symbol $\sigma_{M}^{v}(A)$ is an entire function taking values in $\mathcal{L}\left(H_{p}^{s}\left(Y,\left.E\right|_{Y}\right), H_{p}^{s-v}\left(Y,\left.F\right|_{Y}\right)\right)$ for any $s \in \mathbb{R}, 1<p<\infty$.

Ellipticity of cone-degenerate differential operators is determined by the invertibility of these two symbols, as given in the following definition.

Definition 2.5. [11, 7.1.2, Definition 14] An operator $A \in \operatorname{Diff}_{c}^{v}(X)$ is called cone-elliptic (with respect to a weight $\gamma \in \mathbb{R}$ ) if

1. it is an elliptic differential operator in the interior int $X$ and the compressed principal symbol $\widetilde{\sigma}_{\psi}^{v}(A)$ is invertible on ${ }^{c} T^{*} X \backslash 0$,

2. the operator $\sigma_{M}^{v}(A): H_{p}^{s}\left(Y,\left.E\right|_{Y}\right) \rightarrow H_{p}^{s-v}\left(Y,\left.F\right|_{Y}\right)$ are isomorphisms for some $s=s_{0}$ and all $z \in \Gamma_{\frac{N+1}{p}-\gamma}$, here $\Gamma_{\beta}:=\{z \in \mathbb{C} \mid \mathfrak{R} z=\beta\}$.

Remark 2.6. In [11] the authors considered $L^{2}$ spaces. However, by using results in standard theory of pseudo-differential operators, all statements concerning the action of the conormal symbol $\sigma_{M}^{v}(A)(z)$ between $L^{2}$ spaces can be performed in the setting of $L^{p}$ spaces. We will recall the cone calculus in detail in chapter 4.

The following example is to check ellipticity of the Laplace-Beltrami operator with respect to the cone metric in the example 2.4.

Example 2.7. For a sake of simplicity, we consider $X$ as a straight cone, i.e. $g_{Y}(t)$ is independent of $t$. Then the Laplace-Beltrami operator is given in local coordinates by

$$
\Delta_{g} u=t^{-2}\left\{\left(-t \frac{\partial}{\partial t}\right)^{2}+\left\{-N+1-t G^{-1} \frac{\partial G}{\partial t}\right\}\left(-t \frac{\partial}{\partial t}\right)+\Delta_{g_{Y}}\right\} u,
$$

here $G=\sqrt{\operatorname{det}\left(g_{Y, i j}\right)}$.

The compressed principal symbol of $\Delta_{g}$ is

$$
\widetilde{\sigma}_{\psi}^{2}\left(\Delta_{g}\right)(t, y, \widetilde{\tau}, \eta)=-\widetilde{\tau}^{2}-|\eta|^{2} .
$$

The principal conormal symbol is

$$
\sigma_{c}^{2}\left(\Delta_{g}\right)(z)=z^{2}-(N-1) z+\Delta_{g_{Y}} .
$$

Therefore, $\Delta_{g}$ is cone-elliptic with respect to the weight $\gamma$ when

$$
\frac{N+1}{2}-\gamma \neq \frac{N-1 \pm \sqrt{(N-1)^{2}-4 \lambda_{j}}}{2}, j=0,1,2, \ldots,
$$

where $\lambda_{j}$ are the eigenvalues of $\Delta_{g_{Y}(0)}$ on $Y$. 
Remark 2.8. Let us note the following relation

$$
\left.\sigma_{\psi}^{v}\left(\sigma_{M}^{v}(A)\right)(y, \eta, z)\right|_{z=\beta+\mathbb{1} \tau}=\widetilde{\sigma}_{\psi}^{v}(A)(0, y, \widetilde{\tau}, \eta)
$$

where the principal symbol $\sigma_{\psi}^{v}$ on the left-hand side is the parameter-dependent principal symbol. Therefore, if condition 1) in definition 2.5 is fulfilled, it follows that the conormal symbol $\sigma_{M}^{v}(A)(z)$ is invertible for $z \in \mathfrak{R}_{\beta},|z|$ large enough.

\subsubsection{Function spaces}

In this work, we use constantly the notation of cut-off and excision functions. A cut-off function is a function $\omega(t) \in C_{0}^{\infty}[0,1)$ such that $\omega(t)=1$ when $t \leq \varepsilon$, and $\omega(t)=0$ when $t>a$ for some $0<\varepsilon<a<1$. Given a set $A \in \mathbb{C}$, an $A$-excision function is a function $\chi(z) \in C^{\infty}(\mathbb{C})$ such that $\chi(z)=0$ if $\operatorname{dist}(z, A) \leq 1 / 2$ and $\chi(z)=1$ if $\operatorname{dist}(z, A) \geq 1$.

\subsubsection{Weighted Sobolev spaces $\mathcal{H}_{p}^{s, \gamma}(X ; E)$}

Let $\left(E, X, \pi_{E}\right)$ be a real smooth vector bundle over $X$. Denote by $h_{E}$ a bundle metric on $E$, which is a non-degenerate bilinear map on each fibre $E_{x}, x \in X$. This map induces a norm in each fiber, which we denote by $\|s(x)\|_{E}$ for an element $s(x) \in E_{x}$. Moreover, using this bundle metric we can identify $E$ with its dual bundle $E^{\prime}$. We often drop the notation of bundles when the meaning is clear.

We fix a positive measure $d \mu$ on the stretched manifold $X$, we have the space of $p$ integrable functions on $X$. Now for two sections $s_{1}, s_{2} \in \Gamma^{-\infty}(X ; E)$, their inner product is given by

$$
\left(s_{1}, s_{2}\right)_{E}=\int_{X} h_{E}\left(s_{1}(x), s_{2}(x)\right) d \mu,
$$

where $t(y)$ is the boundary defining function.

Definition 2.9. For $p>1$ and a weight $\gamma \in \mathbb{R}$, the weighted space $\mathcal{H}_{p}^{0, \gamma}(X ; E)$ consists of all sections $u \in \Gamma^{-\infty}(X ; E)$ such that

$$
\|u\|_{\mathcal{H}_{p}^{0, \gamma}(X ; E)}:=\left(\int_{X}\|s(x)\|_{E}^{p} t^{-\gamma p}(y) d \mu\right)^{\frac{1}{p}}<\infty,
$$

For a natural number $m$, the weighted Sobolev space $\mathcal{H}_{p}^{m, \gamma}(X ; E)$ consists of all sections $u$ such that

$$
X_{1} \circ X_{2} \circ \ldots \circ X_{k} u \in \mathcal{H}_{p}^{0, \gamma}(X ; E), \forall k \leq m,
$$

for all smooth vector fields $X_{i}$ on $X$ which are tangent to the boundary $Y$. 
The space $\mathcal{H}_{p}^{s, \gamma}(X ; E)$ for real $s$ is defined by using complex interpolation and duality with respect to the inner product (2.6) of $\mathcal{H}_{2}^{0,0}(X ; E)$. Later we often drop the subscript in any $L^{2}$ base function spaces.

Remark 2.10. Having identified $E \cong E^{\prime}, F \cong F^{\prime}$, the formal adjoint operator $A^{\prime}$ of a cone operator $A \in \operatorname{Diff}_{c}^{\mu}(X ; E, F)$ is determined by the formula

$$
(A u, v)_{F}=\left(u, A^{\prime} v\right)_{E}
$$

for sections $u \in C_{c}^{\infty}(X \backslash Y ; E)$ and $u \in C_{c}^{\infty}(X \backslash Y ; F)$.

The spaces above can alternatively be described in local coordinates as follows.

Definition 2.11. Given a weight $\gamma \in \mathbb{R}, 1<p<\infty, m \in \mathbb{N}_{0}$, the weighted Sobolev space $\mathcal{H}_{p}^{m, \gamma}(X ; E)$ consists of all distributions $u \in H_{p, \text { loc }}^{m}($ int $X ; E)$ such that

$$
t^{-\gamma}(t \partial t)^{k} \partial_{y}^{\alpha}(\omega u)(t, y) \in L_{p}\left(X ; E, t^{N} d t d y\right)
$$

for all $k+|\alpha| \leq m$ in a collar neighborhood of the boundary.

The index $s$ describes the smoothness of these distributions, and the index $\gamma$ describes their flatness, that is how they vanish or blow up when approaching the boundary.

According to the definition above, a function $u \in \mathcal{H}_{p}^{s, \gamma}(X ; E)$ is in the interior of $X$ a function belonging to $H_{p}^{s}(2 X)$, and in a collar neighborhood of the boundary, its $L^{p}$ norm can be written in local coordinates as

$$
\|u\|_{\mathcal{H}_{p}^{0, \gamma}(X)}^{p}=\int_{(0,1] \times Y} t^{N-\gamma p}\|u\|_{E}^{p} d t d y
$$

We recall some important properties of these function spaces, and we write $\mathcal{H}_{p}^{s, \gamma}(X)$ instead of $\mathcal{H}_{p}^{s, \gamma}(X ; E)$ :

a) $\mathcal{H}_{p}^{m, \gamma}(X)$ is a Banach space, and a Hilbert space for $p=2$.

b) When $\gamma=0$, we recover the usual $L^{p}$ space: $\mathcal{H}_{p}^{0,0}(X)=L^{p}(X)$.

c) $\left(\mathcal{H}_{p}^{s, \gamma}(X)\right)^{*} \cong \mathcal{H}_{p^{\prime}}^{-s,-\gamma}(X)$.

d) $t^{\gamma^{\prime}} \mathcal{H}_{p}^{s, \gamma}(X)=\mathcal{H}_{p}^{s, \gamma+\gamma^{\prime}}(X)$.

e) $\mathcal{H}_{p}^{s, \gamma}(X)$ is continuously embedded in $\mathcal{H}_{p}^{s^{\prime}, \gamma^{\prime}}(X)$ when $s \geq s^{\prime}, \gamma \geq \gamma^{\prime}$. This embedding is compact when $s>s^{\prime}, \gamma>\gamma^{\prime}$. 
f) $\mathcal{H}_{p}^{s, \gamma}(X)$ is continuously embedded in $\mathcal{H}_{q}^{r, \gamma^{\prime}}(X)$ when $\gamma-\gamma^{\prime} \geq(N+1)(1 / p-1 / q)$ and $s-r \geq(N+1)(1 / p-1 / q)$. This embedding is compact when these inequalities are strict.

The properties (a-e) are straightforward by definition. We will prove the last property in the next subsection.

\subsubsection{Embedding theorems}

In the following, we need several embedding theorems for weighted Sobolev spaces, namely $\mathcal{H}_{p}^{s, \gamma}(X) \hookrightarrow \mathcal{H}_{q}^{r, \delta}(X)$, where $s \geq r$. We will prove such embeddings in the cases $p \leq q$ and $p>q$. The latter case is easier, by using boundedness of the measure on $X$, and the embeddings are always compact.

Theorem 2.12. Let $1 \leq q<p ; s, r \in \mathbb{N}$ such that $s \geq r$ and

$$
\widetilde{\gamma}-\gamma<(N+1)\left(\frac{1}{q}-\frac{1}{p}\right)
$$

Then it holds

$$
\mathcal{H}_{p}^{s, \gamma}(X) \hookrightarrow \mathcal{H}_{q}^{r, \widetilde{\gamma}}(X)
$$

Proof. By definition,

$$
\mathcal{H}_{p}^{s, \gamma}(X)=\omega \mathcal{H}_{p}^{s, \gamma}((0,1] \times Y)+(1-\omega) H_{p}^{s}(2 X)
$$

We apply the usual Sobolev embedding theorems $H_{p}^{s}(2 X) \hookrightarrow H_{q}^{r}(2 X)$, here $s \geq r, p \geq q$.

It remains to show that $\mathcal{H}_{p}^{s, \gamma}((0,1] \times Y) \hookrightarrow \mathcal{H}_{q}^{r, \widetilde{\gamma}}((0,1] \times Y)$, which we will verify by using expressions in local coordinates and first in the case $s=r=0$.

Indeed, given a $u \in \mathcal{H}_{p}^{0, \gamma}((0,1] \times Y)$, by Hölder inequality we have

$$
\begin{aligned}
\int_{(0,1] \times Y}\left|t^{-\widetilde{\gamma}} u\right|^{q} t^{N} d t d y & =\int_{(0,1] \times Y}\left|t^{-\gamma} u\right|^{q} t^{N q / p} t^{q(\gamma-\widetilde{\gamma})+N(1-q / p)} d t d y \\
& \leq\left(\int\left(\left|t^{-\gamma} u\right|^{q} t^{N q / p}\right)^{p / q} d t d y\right)^{q / p}\left(\int\left(t^{q(\gamma-\widetilde{\gamma})+N(1-q / p)}\right)^{p /(p-q)} d t d y\right)^{(p-q) / p} \\
& \leq\left(\int_{(0,1] \times Y}\left|t^{-\gamma} u\right|^{p} t^{N} d t d y\right)^{q / p}\left(\int_{(0,1] \times Y} t^{p q /(p-q)(\gamma-\widehat{\gamma})} t^{N} d t d y\right)^{(p-q) / p}
\end{aligned}
$$

The second integral is bounded because of the condition 2.7) on $\widetilde{\gamma}-\gamma$. Therefore, we obtain $\mathcal{H}_{p}^{0, \gamma}(X) \hookrightarrow \mathcal{H}_{q}^{0, \tilde{\gamma}}(X)$. We repeat using this embedding for all derivatives $\left(t \partial_{t}\right)^{k} \partial_{y}^{\alpha} u$, 
$k+|\alpha| \leq s$, of a function $u \in \mathcal{H}_{p}^{s, \gamma}(X)$, then we obtain $\mathcal{H}_{p}^{s, \gamma}(X) \hookrightarrow \mathcal{H}_{q}^{s, \widetilde{\gamma}}(X)$. Finally, by using the embeddings of intermediate spaces

$$
\mathcal{H}_{p}^{s, \gamma}(X) \hookrightarrow \mathcal{H}_{q}^{s, \widetilde{\gamma}}(X) \hookrightarrow \mathcal{H}_{q}^{r, \widetilde{\gamma}}(X)
$$

we conclude the general embedding.

Moreover, the embedding $\mathcal{H}_{p}^{s, \gamma}(X) \hookrightarrow \hookrightarrow \mathcal{H}_{q}^{r, \widetilde{\gamma}}(X)$ is compact because of the strict inequality on $\widetilde{\gamma}$ and the results [29, Theorem 7.6].

In the case $p \leq q$, one needs the Hardy inequality in one dimension.

Lemma 2.13 ([29, Example 6.8]). Let $1 \leq p \leq q<\infty$. Let $f=f(t) \in A C_{R}((0,1))$ be a function defined and differentiable almost everywhere on $(0, \infty)$ such that $\lim _{t \rightarrow \infty} f(t)=0$. Then the following inequality holds

$$
\left(\int_{0}^{1}|f(t)|^{q} t^{\alpha} d t\right)^{1 / q} \leq C\left(\int_{0}^{1}\left|f^{\prime}(t)\right|^{p} t^{\beta} d t\right)^{1 / p}
$$

if and only if either $\beta>p-1, \alpha \geq \beta \frac{q}{p}-\frac{q}{p^{\prime}}-1$, or $\beta \leq p-1, \alpha>-1$.

The embedding is as follows.

Theorem 2.14. Let $1<p \leq q<\infty$. Then $\mathcal{H}_{p}^{s, \gamma}(X) \hookrightarrow \mathcal{H}_{q}^{r, \widetilde{\gamma}}(X)$ provided that

$$
s-r \geq(N+1)\left(\frac{1}{p}-\frac{1}{q}\right),
$$

and either $\gamma \geq \frac{(N+1)}{p}, \widetilde{\gamma}<\frac{(N+1)}{q}$, or $\gamma<\frac{(N+1)}{p}, \gamma-\widetilde{\gamma} \geq(N+1)\left(\frac{1}{p}-\frac{1}{q}\right)$.

Moreover, the embedding is compact if and only if all inequalities above are strict.

Proof. We follow the proof of theorem 2.12, The embedding $H_{p}^{s}(2 X) \hookrightarrow H_{q}^{r}(2 X)$ holds due to the condition $s-r \geq(N+1)(1 / p-1 / q)$. This embedding is compact when this inequality is strict.

We need to show that $\mathcal{H}_{p}^{s, \gamma}((0,1] \times Y) \hookrightarrow \mathcal{H}_{q}^{r, \widetilde{\gamma}}((0,1] \times Y)$. We prove first the case $s=1, r=0$. Particularly, the embedding holds provided that the following inequality is true for an arbitrary function $u \in \mathcal{H}_{p}^{1, \gamma}((0,1] \times Y)$ :

$$
\left(\int_{(0,1] \times Y}\left|t^{-\widetilde{\gamma}} u\right|^{q} d \mu\right)^{1 / q} \leq C\left(\int_{(0,1] \times Y}\left|t^{1-\gamma} \partial_{t} u\right|^{p} d \mu\right)^{1 / p} .
$$

Indeed, using the one dimensional Hardy inequality, we have

$$
\left(\int_{0}^{1} t^{N-q \widetilde{\gamma}}|u|^{q} d t\right)^{1 / q} \leq C\left(\int_{0}^{1} t^{N-p \gamma}\left|\partial_{t} u\right|^{p} d t\right)^{1 / p},
$$


where either $\gamma \geq \frac{(N+1)}{p}, \widetilde{\gamma}<\frac{(N+1)}{q}$, or $\gamma<\frac{(N+1)}{p}, \gamma-\widetilde{\gamma} \geq(N+1)\left(\frac{1}{p}-\frac{1}{q}\right)$.

Integrating both sides over $Y$, then we obtain the desired embedding.

For general $s, r$, we repeat utilizing the embedding above for intermediate spaces

$$
\mathcal{H}_{p}^{s, \gamma}(X) \hookrightarrow \mathcal{H}_{p_{1}}^{s-1, \delta_{1}}(X) \hookrightarrow \ldots \hookrightarrow \mathcal{H}_{p_{i}}^{s-i, \delta_{i}}(X) \hookrightarrow \ldots \hookrightarrow \mathcal{H}_{q}^{r, \widetilde{\gamma}}(X),
$$

where for $i=1, \ldots, s-r-1, p_{0}=p, p_{s-r}=q, \delta_{0}=\gamma, \delta_{s-r}=\widetilde{\gamma}$, other indices are determined by the formulae

$$
(N+1)\left(\frac{1}{p_{i}}-\frac{1}{p_{i+1}}\right)=\delta_{i}-\delta_{i+1} \leq 1,(N+1)\left(\frac{1}{r_{s-r+1}}-\frac{1}{q}\right) \leq \delta-\delta_{s-r} .
$$

The compactness of those embeddings are verified by using compact embedding theorems for usual Sobolev spaces and compactness of Hardy operators, see [29, Theorem 7.6].

\subsubsection{Mapping properties of cone-degenerate differential operators}

We recall mapping properties of cone-degenerate differential operators when they act as bounded operators between the scale of cone Sobolev spaces. Following is the statements about continuity.

Theorem 2.15 ([31, Proposition 2.13]). A v-order cone-degenerate differential operator $A \in \operatorname{Diff}_{c}^{v}(X)$ induces continuous mappings

$$
A: \mathcal{H}_{p}^{s, \gamma}(X) \rightarrow \mathcal{H}_{p}^{s-v, \gamma-\gamma}(X),
$$

for all $s, \gamma \in \mathbb{R}$.

Ellipticity in the sense 4.22 is equivalent to Fredholm property of the operator acting between weighted Sobolev spaces and the existence of parametrices. The notion of parametrices for cone-differential operators is stated in [31, 7.1.2, Definition 15] when $p=2$, which can be generalized in the $L^{p}$ spaces as follows.

Definition 2.16. An operator

$$
P \in \bigcap_{s \in \mathbb{R}} \mathcal{L}\left(\mathcal{H}_{p}^{s, \gamma-\gamma}(X), \mathcal{H}_{p}^{s+v, \gamma}(X)\right)
$$

is called a parametrix for $A \in \operatorname{Diff}_{c}^{v}(X)$ with respect to a fixed weight $\gamma \in \mathbb{R}$, if there is an $\varepsilon>0$ such that

$$
\begin{aligned}
& A P-I \in \bigcap_{s \in \mathbb{R}} \mathcal{L}\left(\mathcal{H}_{p}^{s, \gamma-\gamma}(X), \mathcal{H}_{p}^{\infty, \gamma-v+\varepsilon}(X)\right), \\
& P A-I \in \bigcap_{s \in \mathbb{R}} \mathcal{L}\left(\mathcal{H}_{p}^{s, \gamma}(X), \mathcal{H}_{p}^{\infty, \gamma+\varepsilon}(X)\right) .
\end{aligned}
$$


We state now the elliptic regularity theorem.

Theorem 2.17 ([31, Corollaries 3.3, 3.4]). The following conditions for $A \in \operatorname{Diff}_{c}^{v}(X)$ are equivalent:

1. $A: \mathcal{H}_{p}^{s, \gamma}(X) \rightarrow \mathcal{H}_{p}^{s-v, \gamma-\gamma}(X)$ is a Fredholm operator for a certain $s \in \mathbb{R}$ (and then for all $s \in \mathbb{R})$,

2. A is elliptic with respect to the weight $\gamma$.

If $A$ is elliptic with respect to the weight $\gamma$, then there exists a parametrix for A. Furthermore,

$$
A u=f \in \mathcal{H}_{p}^{s-v, \gamma-v}(X) \text { for some } s \in \mathbb{R}
$$

and

$$
u \in \mathcal{H}_{p}^{-\infty, \gamma}(X) \text { imply } u \in \mathcal{H}_{p}^{s, \gamma}(X) .
$$

Even more, if $f \in \mathcal{H}_{p, Q}^{s-v, \gamma-v}(X)$, then $u \in \mathcal{H}_{p, P}^{s, \gamma}(X)$ for a certain asymptotic type $P=P(A, Q)$ depending only on $A$ and $Q$.

Remark 2.18. As in the standard case, cone-degenerate differential operators are special elements of an algebra of cone-degenerate pseudo-differential operators. Those aforementioned results have their generalizations in the cone calculus which we will present in chapter 4. We also note that there is another scale of so-called cone Sobolev spaces with asymptotics, which will provide additional asymptotic information. The full calculus involving such spaces will be essential in regularity problem.

\subsection{Existence theorems for monotone operators}

Our approach to show existence is to reduce the observed partial differential equations to operator equations and use tools from nonlinear functional analysis, namely theory of monotone operators and topological methods to solve the reduced ones.

The present section collects two existence theorems in the theory of monotone operators. The point in common is that they are applied to equations involving coercive operators. Let us recall some fundamental definitions.

Given a Banach space $X$ and a subset $D \subseteq X$, we denote by $X^{*}$ its dual, by $\langle\cdot, \cdot\rangle$ the pairing of $X$ and $X^{*}$, and by $\rightarrow, \rightarrow$ the strong and weak convergence in a Banach space, respectively. 
Definition 2.19 ([38, Chapter 1, Definition 1.2]). Given a mapping $T: \bar{D} \subseteq X \rightarrow X^{*}$.

- $T$ is said to be demi-continuous on $D$, if for any sequence $u_{n} \in D$ strongly converging to $u_{0} \in D$, it holds $T u_{n} \rightarrow T u_{0}$ in $X^{*}$, or equivalently

$$
\lim _{n \rightarrow \infty}\left\langle T u_{n}, v\right\rangle=\left\langle T u_{0}, v\right\rangle, \forall v \in X
$$

- $T$ is said to be bounded if it carries bounded subsets of $D$ into bounded subsets of $X^{*}$.

- $T$ is said to be coercive if

$$
\lim _{\|u\|_{X} \rightarrow \infty} \frac{\langle T u, u\rangle}{\|u\|_{X}}=\infty .
$$

Definition 2.20 ([38, Chapter 1, Definition 1.1]). An operator

$$
T: D \subseteq X \rightarrow X^{*}
$$

is said to be monotone, if the inequality

$$
\langle T u-T v, u-v\rangle \geq 0
$$

holds for arbitrary $u, v \in D$.

Now we recall the Browder theorem.

Theorem 2.21 ([10, Theorem 5.3.2]). Let $X$ be a reflexive real Banach space. Moreover, let $T: X \rightarrow X^{*}$ be an operator satisfying the conditions

(i) $T$ is bounded,

(ii) $T$ is demi-continuous,

(iii) $T$ is coercive,

(iv) $T$ is monotone on the space $X$.

Then the equation $T v=f^{*}$ has at least one solution $v \in X^{*}$ for every $f^{*} \in X^{*}$.

An extension of this theorem was contributed by Leray and Lions, when the monotonicity condition of the whole operator is relaxed.

Theorem 2.22 ([10, Theorem 5.3.3]). Let X be a reflexive real Banach space. Moreover, let $T: X \rightarrow X^{*}$ be an operator satisfying the conditions 
(i) $T$ is bounded,

(ii) $T$ is demi-continuous,

(iii) $T$ is coercive.

Moreover, let there exist a bounded mapping $\Phi: X \times X \rightarrow X^{*}$ such that

(iv) $\Phi(u, u)=T(u)$ for every $u \in X$,

(v) for all $u, w$, and $h \in X$ and any sequence $\left\{t_{n}\right\}_{n \geq 1}$ of real numbers such that $t_{n} \rightarrow 0$, we have

$$
\Phi\left(u+t_{n} h, w\right) \rightarrow \Phi(u, w) \geq 0,
$$

(vi) for all $u, w \in X$ we have

$$
\langle\Phi(u, u)-\Phi(w, u), u-w\rangle \geq 0
$$

(vii) if $u_{n} \rightarrow u$ and

$$
\lim _{n \rightarrow \infty}\left\langle\Phi\left(u_{n}, u_{n}\right)-\Phi\left(u, u_{n}\right), u_{n}-u\right\rangle=0
$$

then we have

$$
\Phi\left(w, u_{n}\right) \rightarrow \Phi(w, u) \text { for arbitrary } w \in X
$$

(viii) If $w \in X, u_{n} \rightarrow u$, $\Phi\left(w, u_{n}\right) \rightarrow z$, then

$$
\lim _{n \rightarrow \infty}\left\langle\Phi\left(w, u_{n}\right), u_{n}\right\rangle=\langle z, u\rangle
$$

Then the equation $T v=f^{*}$ has at least one solution $v \in X^{*}$ for every $f^{*} \in X^{*}$.

These theorems are particularly powerful to be applied to quasilinear equations in divergence form where the nonlinearities are themselves monotone, because these equations can be reduced to operator equations where the induced operator possesses a kind of monotonicity. We notice that these two theorems require a coercive condition on the operator, however, the Leray-Lions theorem requires only monotonicity in the principal part, namely property (vi). In the next section, we recall the mapping degree method, which is helpful also when dealing with noncoercive operators. 


\subsection{Mapping degree}

Mapping degree theory started with the so-called Brouwer degree constructed for finite dimensional mappings. It is determined uniquely by three axioms including normalization, additivity on a domain and invariance under homotopies. The first generalization to mappings between Banach spaces was developed by Leray-Schauder in 1934 (see [25]), where the authors also introduced a way to reduce a nonlinear boundary value problem to an operator equation of the form $(I-F) u=0$. In the case of quasilinear Dirichlet problem, the operator $F$ is compact. However, the Neumann problem yields a noncompact operator, to which situation the Leray-Schauder degree theory is not applicable. At the end of the sixties, Browder and Skrypnik independently constructed the degree for more general monotone mappings, namely operators of class $(S)_{+}$(named by Browder) or operators having $\alpha$ property (named by Skrypnik). This mapping degree not only helps solving other boundary value problems, but also relaxes conditions of the coefficients involved. As mentioned in the introduction of this thesis, this mapping degree gives a simple sufficient criterion for solvability, which covers the class of coercive and odd operators. In this section, the degree theory for mappings having property $\alpha$ and some general theorems for existence of solutions to operator equations are summarized. For deeper understanding of the theory, we refer to the monographs [37, 38] for systematic investigation and various applications to solving nonlinear boundary value problems.

\subsubsection{Generalized monotone mappings}

In the following, $X$ is a real separable reflexive Banach space. First, we will recall some basic definitions concerning monotonicity.

Definition 2.23 ([38, Chapter 2, Definitions 2.1,2.2]). We say that

a) an operator $T: F \rightarrow X^{*}, F \subseteq X$ is said to satisfy the condition $\alpha_{0}(F)$ if for any sequence $u_{n} \in F, u_{n} \rightarrow u_{0}, T u_{n} \rightarrow 0$, and

$$
\limsup _{n \rightarrow \infty}\left\langle T u_{n}, u_{n}-u_{0}\right\rangle \leq 0
$$

then $u_{n} \rightarrow u$.

b) an operator $T: \bar{D} \rightarrow X^{*}, D$ is a bounded, open subset of $X$, is said to satisfy the condition $\alpha(F), F \subset \bar{D}$, if for any sequence $u_{n} \in F, u_{n} \rightarrow u_{0}$, and

$$
\limsup _{n \rightarrow \infty}\left\langle T u_{n}, u_{n}-u_{0}\right\rangle \leq 0
$$


then $u_{n} \rightarrow u$.

Remark 2.24. The condition $(S)_{+}$coincides with the condition $\alpha(\bar{D})$. This property is stable under compact perturbation. This fact is easily verified by definition, however, it is of importance when we deal with equations with lower order terms which induce compact perturbations and the principal parts correspond to operators having $(S)_{+}$property.

\subsubsection{Degree of generalized monotone mappings}

In this part, we summarize some results on the construction of degree of generalized monotone mappings. The main reference is the monograph of Skrypnik ([38] $)$. For more details in the development of degree theory, we refer to the book by Drábek and Milota ([10]).

The mapping degree was first defined by Brouwer for finite-dimensional mappings, we recall an axiomatic definition.

Definition 2.25 ([38, Chapter 2]). Given a continuous mapping $f: \bar{\Omega} \rightarrow \mathbb{R}^{n}, \Omega$ is a bounded subset in $\mathbb{R}^{n}, f(x)=\left(f_{1}\left(x_{1}, \ldots, x_{n}\right), \ldots, f_{n}\left(x_{1}, \ldots, x_{n}\right)\right)$. Assume additionally that $f(x) \neq 0$ for $x \in \partial \Omega$. Then we can assign an integer-valued characteristic $\operatorname{deg}(f, \bar{\Omega}, 0)$ to $f$, which is uniquely determined by the following properties:

1. If $f(x)=x-x_{0}$, where $x_{0} \in \Omega$, then $\operatorname{deg}(f, \bar{\Omega}, 0)=1$.

2. If $\Omega_{1}, \Omega_{2}$ are disjoint open subsets of $\Omega$ and $f(x) \neq 0$ for $x \in \bar{\Omega} \backslash\left(\Omega_{1} \cup \Omega_{2}\right)$, then

$$
\operatorname{deg}(f, \bar{\Omega}, 0)=\operatorname{deg}\left(f, \bar{\Omega}_{1}, 0\right)+\operatorname{deg}\left(f, \bar{\Omega}_{2}, 0\right) .
$$

3. If $h:[0,1] \times \bar{\Omega} \rightarrow \mathbb{R}^{n}$ is a continuous mapping such that $h(x, t) \neq 0$ for $t \in[0,1]$, $x \in \partial \Omega$, and

$$
f_{0}=h(0, x), f_{1}(x)=h(1, x), x \in \bar{\Omega},
$$

then $\operatorname{deg}\left(f_{0}, \bar{\Omega}, 0\right)=\operatorname{deg}\left(f_{1}, \bar{\Omega}, 0\right)$.

Now the degree $\operatorname{Deg}(T, \bar{D}, 0)$ of a mapping $T$ on the set $\bar{D}$ with respect to the origin of the space $X^{*}$ can be introduced under the conditions:

a) $T \in A_{0}(D, \partial D)$ the set of all bounded demi-continuous mappings from $\bar{D}$ to $X^{*}$ satisfying condition $\alpha_{0}(\partial D)$, 
b) $T u \neq 0$ for any element $u \in \partial D$.

Assume that $\left\{v_{i}\right\}$ is a complete linearly independent system of the separable space $X$, and denote by $F_{n}$ the linear hull of the elements $v_{1}, v_{2} \ldots v_{n}$. We define for $n=1,2, \ldots$ the finite-dimensional approximation $T_{n}$ of $T$ as follows

$$
T_{n} u=\sum_{i=1}^{n}\left\langle T u, v_{i}\right\rangle v_{i} \text { for } u \in \overline{D \cap F_{n}} .
$$

The following theorem will give us the definition of the degree of $T$.

Theorem 2.26 ([38, Chapter 2, Theorems 2.1, 2.2]). Let $T$ be an operator satisfying conditions a), b) above. Then there exists an $N_{0} \in \mathbb{N}$ such that for $n \geq N_{0}$ the following assertions hold:

1. the equation $T_{n} u=0$ has no solutions belonging to $\partial D_{n}$;

2. the degree $\operatorname{deg}\left(T_{n}, \overline{D_{n}}, 0\right)$ is defined and independent of $n$.

Furthermore, the limit

$$
D v_{i}=\lim _{n \rightarrow \infty} \operatorname{deg}\left(T_{n}, \overline{D_{n}}, 0\right)
$$

does not depend on the choice of the sequence $\left\{v_{i}\right\}$.

Definition 2.27 ([38, Chapter 2, Definition 2.4]). To an operator $T$ satisfying conditions a), b) above we can assign the so-called degree of $T$ on the set $\bar{D}$ with respect to the point $0 \in X^{*}$ by

$$
\operatorname{Deg}(T, \bar{D}, 0):=\lim _{n \rightarrow \infty} \operatorname{deg}\left(T_{n}, \overline{D_{n}}, 0\right)
$$

For detailed construction of this mapping degree, see Appendix A. Moreover, this topological characteristic can be constructed for generalized monotone operators acting on a non-separable space, we refer the readers to the monograph of Skrypnik ([38]).

\subsubsection{Properties of the mapping degree}

The mapping degree of generalized monotone operators possesses all the properties of the degree of finite-dimensional mappings. In particular, it is homotopy invariant among this class of operators, namely $A_{0}(D, \partial D)$. The homotopy invariance is understood in the following sense. 
Definition 2.28 ([38, Chapter 2, Definitions 4.1, 4.2]). An operator family

$$
T_{t}: \bar{D} \times[0,1] \rightarrow X^{*}
$$

is said to satisfy the condition $\alpha_{0}^{(t)}(\partial D)$, if for any sequences $u_{n} \in \partial D$ and $t_{n} \in[0,1]$ such that $u_{n} \rightarrow u_{0}, T_{t_{n}}\left(u_{n}\right) \rightarrow 0$, and

$$
\lim _{n \rightarrow \infty}\left\langle T_{t_{n}}\left(u_{n}\right), u_{n}-u_{0}\right\rangle=0
$$

then $u_{n}$ converges strongly to $u_{0}$.

Let $T^{\prime}, T^{\prime \prime}: \bar{D} \rightarrow X^{*}$ be mappings of class $A_{0}(D, \partial D)$ and let $T^{\prime} u \neq 0, T^{\prime \prime} u \neq 0$ for $u \in \partial D$. The mappings $T^{\prime}$ and $T^{\prime \prime}$ are said to be homotopic on $\bar{D}$ if there is a oneparameter family of mappings $T_{t}: \bar{D} \rightarrow X^{*}, t \in[0,1]$ satisfying condition $\alpha_{0}^{(t)}(\partial D)$ such that

a) $T_{t} u \neq 0$ for $u \in \partial D, t \in[0,1]$ and $T_{0}=T^{\prime}, T_{1}=T^{\prime \prime}$.

b) for any sequences $\left\{u_{n}\right\} \subset \bar{D},\left\{t_{n}\right\} \subset[0,1]$ are such that $t_{n} \rightarrow t_{0}, u_{n} \rightarrow u_{0}$, the sequence $T_{t_{n}} u_{n}$ converges weakly to $T_{t_{0}} u_{0}$.

Two operators which are homotopic in this sense will have equal degrees.

Theorem 2.29 ([38, Chapter 2, Theorem 4.1]). Let $T^{\prime}, T^{\prime \prime}: \bar{D} \rightarrow X^{*}$ be two mappings of class $A_{0}(D, \partial D)$. Suppose that $T^{\prime} u \neq 0, T^{\prime \prime} u \neq 0$ for $u \in \partial D$ and that the mappings $T^{\prime}, T^{\prime \prime}$ are homotopic on $\bar{D}$. Then

$$
\operatorname{Deg}\left(T^{\prime}, \bar{D}, 0\right)=\operatorname{Deg}\left(T^{\prime \prime}, \bar{D}, 0\right)
$$

\subsubsection{Existence theorems}

The mapping degree defined above gives an important and simple criterion to determine whether a mapping is surjective, as stated in the following theorem.

Theorem 2.30 ([38, Chapter 2, Corollary 4.1]). Let $T: \bar{D} \rightarrow X^{*}$ be a mapping of class $A_{0}(D)$ and let $T u \neq 0$ for $u \in \partial D$. In order that the equation $T u=0$ has a solution in $\bar{D}$, it is sufficient that $\operatorname{Deg}(T, \bar{D}, 0) \neq 0$.

Although this criterion is simple, it is helpful in extending the class of solvable equations. Indeed, because the general mapping degree is constructed by approximation with finite-dimensional operators, by choosing and calculating the degrees of those approximations, we can calculate the mapping degree of specific operators, namely coercive and odd operators, as in the following lemma. 
Lemma 2.31 ([38, Chapter 2, Theorems 4.4, 4.5]). a) Let $T: \bar{D} \rightarrow X^{*}$ be a mapping of class $A_{0}(D, \partial D)$. Suppose that $0 \in \bar{D} \backslash \partial D$ and for $u \in \partial D$

$$
T u \neq 0,\langle T u, u\rangle \geq 0 .
$$

Then $\operatorname{Deg}(T, \bar{D}, 0)=1$.

b) Let $B_{R}(0)=\left\{u \in X:\|u\|_{X} \leq R\right\}$ and let $T: \overline{B_{R}(0)} \rightarrow X^{*}$ be a mapping of class $A\left(B_{R}, \partial B_{R}\right)$. Suppose that for $u \in \partial B_{R}, T u \neq 0$ and

$$
\frac{T u}{\|T u\|_{*}} \neq \frac{T(-u)}{\|T(-u)\|_{*}} \text {. }
$$

Then $\operatorname{Deg}\left(T, \overline{B_{R}}, 0\right)$ is an odd number.

Using this lemma, the existence holds in cases of coercive and odd operators.

Besides that, utilizing the homotopy invariance property of the mapping degree, another approach is to construct a homotopy with a reference operator, whose mapping degree is known in advance to be nonzero. We have the second existence theorem:

Theorem 2.32 ([38, Chapter 2, Theorem 7.1]). Let $X$ be a separable reflexive Banach space, $D$ be a bounded domain in $X$ with boundary $\partial D$ and $T: \bar{D} \times[0,1] \rightarrow X^{*}$ be a bounded demi-continuous operator, such that

1. the family of operators $T_{t}=T(\cdot, t)$ satisfies the condition $\alpha_{0}^{(t)}(\partial D)$ and the operator $T_{1}$ satisfies the condition $\alpha_{0}(\bar{D})$,

2. $T(u, t) \neq 0$ for $u \in \partial D, t \in[0,1]$,

3. $\operatorname{Deg}\left(T_{0}, \bar{D}, 0\right) \neq 0$.

Then the equation $T_{1} u=0$ has at least one solution in $D$.

Combining this theorem and the results on the mapping degree of coercive and odd operators, we obtain further existence theorem.

Theorem 2.33 ([38, Chapter 2, Theorem 7.2]). Let $X$ be a separable reflexive Banach space, $t \in[0,1], T_{t}: X \rightarrow X^{*}$ be a family of bounded demi-continuous operators satisfying condition $\alpha(X)$ and such that, for any bounded set $B \subset X, T_{t}(u)$ depends continuously on $t$, uniformly with respect to $u \in B$. Suppose that condition 1) as well as one of the three conditions 2a)-2c) are fulfilled. 
1) there is a continuous functional $R: X \rightarrow \mathbb{R}$ such that $T_{t} u=h, t \in[0,1]$, imply $\|u\|<R(h)$

2a) the operator $T_{0}$ is coercive,

2b) the operator $T_{0}$ is odd,

2c) $X$ and $X^{*}$ are uniformly convex spaces and

$$
\lim _{\|u\| \rightarrow \infty}\left\{\left\|T_{0} u\right\|_{*}+\frac{\left\langle T_{0} u, u\right\rangle}{\|u\|}\right\}=+\infty .
$$

Then the equation $T_{1} u=h$ is solvable for any $h \in X^{*}$.

Let us illustrate the case of an odd operator by an example.

Example 2.34. [10, Example 5.2.21] Consider the boundary value problem

$$
\left\{\begin{array}{l}
-\left(|\dot{x}(t)|^{p-2} \dot{x}(t)\right)-g(x(t))=f(t), t \in(0,1), \\
x(0)=x(1)=0
\end{array}\right.
$$

where $p>1, f \in L^{p^{\prime}}(0,1)$, and $g: \mathbb{R} \rightarrow \mathbb{R}$ is a continuous function. We denote by $0<\lambda_{1}<\lambda_{2}<\ldots, \lim _{n \rightarrow \infty} \lambda_{n}=\infty$ are eigenvalues of the eigenvalue problem

$$
\left\{\begin{array}{l}
-\left(|\dot{x}(t)|^{p-2} \dot{x}(t)\right)-\lambda|x(t)|^{p-2} x(t)=f(t), t \in(0,1), \\
x(0)=x(1)=0 .
\end{array}\right.
$$

We assume further that

$$
\lim _{s \rightarrow \pm \infty} \frac{g(s)}{|s|^{p-2} s}=\lambda, \text { where } \lambda_{n}<\lambda<\lambda_{n+1} \text { for some } n \in \mathbb{N} .
$$

Denote by $X$ the space $W_{0}^{1, p}(0,1)$ furnished with the seminorm

$$
\|x\|=\left(\int_{0}^{1}|\dot{x}(t)|^{p} d t\right)^{1 / p} .
$$

We already know that this seminorm is an equivalent norm in $W_{0}^{1, p}(0,1)$.

Let us define operators $J, G: X \rightarrow X^{*}$ and an element $f^{*} \in X^{*}$ by

$$
\begin{aligned}
\langle J x, y\rangle & =\int_{0}^{1}|\dot{x}(t)|^{p-2} \dot{x}(t) \dot{y}(t) d t, \\
\langle G x, y\rangle & =\int_{0}^{1} g(x(t)) y(t) d t, \\
\left\langle f^{*}, y\right\rangle & =\int_{0}^{1} f(t) y(t) d t, \text { for } x, y \in X .
\end{aligned}
$$


Then the equation (2.8) is reduced to the operator equation $J x-G x=f^{*} \in X^{*}$.

We define further an operator $S: X \rightarrow X^{*}$ by

$$
\langle S x, y\rangle=\int_{0}^{1}|x(t)|^{p-2} x(t) y(t) d t \text { for } x, y \in X .
$$

Constructing a homotopy

$$
T_{\tau} x=J x-(1-\tau) G x-\tau \lambda S x+(\tau-1) f^{*}
$$

for $(\tau, x) \in[0,1] \times X$.

Then it is shown that for a large enough number $R>0, T_{\tau} x \neq 0$ for all $\tau \in[0,1]$ and $x \in \partial B(0, R)$. Applying theorem 2.29, it implies

$$
\operatorname{Deg}\left(J-G-f^{*}, B(0, R), 0\right)=\operatorname{Deg}(J-\lambda S, B(0, R), 0) .
$$

The degree on the right-hand side is an odd number by lemma 2.31, as we know $J$ and $S$ are obviously odd operators in $B(0, R)$. Hence, $\operatorname{Deg}\left(J-G-f^{*}, B(0, R), 0\right)$ is also an odd number. Therefore, the equation $J x-G x=f^{*}$ has at least one solution in $x \in B(0, R)$.

\subsection{Inequalities for vectors}

In the study of the $p$-Laplacian, we will need upper and lower bounds of the expression

$$
\left\langle|\nabla u|^{p-2} \nabla u-|\nabla v|^{p-2} \nabla v, \nabla u-\nabla v\right\rangle .
$$

Expressing the gradient in local coordinates, it is an estimate for vectors in $\mathbb{R}^{N+1}$. We will use the following helpful inequalities.

Lemma 2.35. For vectors $a, b \in \mathbb{R}^{N+1}$, one has

1. If $p \geq 2$, then

$$
\left\langle|b|^{p-2} b-|a|^{p-2} a, b-a\right\rangle \geq 2^{-1}\left(|b|^{p-2}+|a|^{p-2}\right)(b-a)^{2} .
$$

If $1<p \leq 2$, then

$$
\left\langle|b|^{p-2} b-|a|^{p-2} a, b-a\right\rangle \leq 2^{-1}\left(|b|^{p-2}+|a|^{p-2}\right)(b-a)^{2} .
$$


2. We have

$$
\begin{aligned}
& \min \{p-1,1\}|b-a|^{2} \int_{0}^{1} \mid a+\left.t(b-a)\right|^{p-2} d t \leq\left\langle|b|^{p-2} b-|a|^{p-2} a, b-a\right\rangle \\
& \leq \max \{p-1,1\}|b-a|^{2} \int_{0}^{1}|a+t(b-a)|^{p-2} d t .
\end{aligned}
$$

Consequently, it holds

$$
\left\langle|b|^{p-2} b-|a|^{p-2} a, b-a\right\rangle \geq 0 .
$$

3. For $p \geq 0$,

$$
|a+b|^{p} \leq C(p)\left(|a|^{p}+|b|^{p}\right)
$$

Proof. The inequalities (2.10) and (2.11) are just consequences of the identity

$$
\left\langle|b|^{p-2} b-|a|^{p-2} a, b-a\right\rangle=\frac{\left(|b|^{p-2}+|a|^{p-2}\right)|b-a|^{2}}{2}+\frac{\left(|b|^{p-2}-|a|^{p-2}\right)\left(|b|^{2}-|a|^{2}\right)}{2}
$$

The inequality (2.12) is shown from the equality

$$
\begin{aligned}
|b|^{p-2} b-|a|^{p-2} a= & \int_{0}^{1} \frac{d}{d t}|a+t(b-a)|^{p-2}(a+t(b-a)) d t \\
= & (b-a) \int_{0}^{1}|a+t(b-a)|^{p-2} d t+ \\
& +(p-2) \int_{0}^{1}|a+t(b-a)|^{p-4}\langle a+t(b-a), b-a\rangle(a+t(b-a)) d t
\end{aligned}
$$

Hence,

$$
\begin{aligned}
\left\langle|b|^{p-2} b-|a|^{p-2} a, b-a\right\rangle & =(b-a)^{2} \int_{0}^{1}|a+t(b-a)|^{p-2} d t \\
& +(p-2) \int_{0}^{1}|a+t(b-a)|^{p-4}\langle a+t(b-a), b-a\rangle^{2} d t .
\end{aligned}
$$

Moreover,

$$
0 \leq \int_{0}^{1}|a+t(b-a)|^{p-4}\langle a+t(b-a), b-a\rangle^{2} d t \leq|b-a|^{2} \int_{0}^{1}|a+t(b-a)|^{p-2} d t .
$$

Consequently, one obtains (2.12).

To verify the inequality (2.14), it suffices to prove for one dimensional case because

$$
|a+b|^{p}=\left(|a|^{2}+|b|^{2}+2 a \cdot b\right)^{p / 2} \leq 2^{\frac{p}{2}}\left(|a|^{2}+|b|^{2}\right)^{p / 2}
$$

This result is obtained by investigating real function of one variable, one can show (2.14), where $C(p)=\max 2^{p / 2}\left\{2^{p-1}, 1\right\}$. 
Lemma 2.36. For $p>1$ and vectors $z, w \in \mathbb{R}^{n}$, the inequality

$$
\int_{0}^{1}|(1-t) z+t w|^{p-2} d t \geq C(|z|+|w|)^{p-2}
$$

holds for some positive constant $C$.

Consequently, when $p \geq 2$ we have

$$
\begin{aligned}
& \left\langle|b|^{p-2} b-|a|^{p-2} a, b-a\right\rangle \geq C(p)|b-a|^{2}\left(|a|^{p-2}+|b|^{p-2}\right), \\
& \left\langle|b|^{p-2} b-|a|^{p-2} a, b-a\right\rangle \geq C(p)|b-a|^{p} .
\end{aligned}
$$

Proof. This inequality is trivial when $1<p \leq 2$, as we have $|(1-t) w+t z| \leq|z|+|w|$. We obtain (2.15) for $C=1$.

In the case $p>2$, when at least one of $|z|=0$ or $|w|=0$, the inequality holds obviously for $C \leq 1 / p-1$.

Now we consider $|z| \neq 0$ and $|w| \neq 0$. If $\left(1-t_{0}\right) z+t_{0} w=0$ for some $t_{0} \in(0,1)$, then we calculate both sides of $(2.15)$, divide them by $|w|^{p-2}$ and show that the left-hand side is

$$
\int_{0}^{1} \frac{\left|t-t_{0}\right|^{p-2}}{\left|1-t_{0}\right|^{p-2}} d t=\frac{1-t_{0}}{p-1}+\frac{t_{0}}{p-1}\left(\frac{t_{0}}{1-t_{0}}\right)^{p-1} \geq \frac{1}{2(p-1)},
$$

which is obvious as $t_{0} \in(0,1)$.

In the remaining case, $z$ and $w$ are linearly independent, the vector $(1-t) z+t w$ never vanishes when $t \in[0,1]$. We introduce new variables

$$
\xi=\frac{z}{|z|+|w|}, \eta=\frac{w}{|z|+|w|}
$$

So $|\xi|+|\eta|=1$. We consider the left-hand side as a function of $(\xi, \eta)$ on the set

$$
C=\left\{(\xi, \eta) \in \mathbb{R}^{2(N+1)}|| \xi|+| \eta \mid=1\right\} .
$$

$C$ is a bounded set in a finite-dimensional space, so it is also compact. As $p>2$, the function

$$
\int_{0}^{1}|(1-t) \xi+t \eta|^{p-2} d t
$$

is continuous on $C$. Therefore, this function attains its minimum on this set, namely at $\left(\xi_{0}, \eta_{0}\right)$. Furthermore, the minimal value is

$$
C_{0}=\int_{0}^{1}\left|(1-t) \xi_{0}+t \eta_{0}\right|^{p-2} d t
$$

If $C_{0}=0$, then $\left|(1-t) \xi_{0}+t \eta_{0}\right|=0$ for all $t \in[0,1]$. Hence, $\xi_{0}=\eta_{0}=0$, which is a contradiction. So $C_{0}>0$ and substituting $z, w$ in place of $\xi, \eta$ gives us

$$
\int_{0}^{1}|(1-t) z+t w|^{p-2} d t \geq C(|z|+|w|)^{p-2} .
$$





\section{Second-order quasilinear elliptic equations}

In this chapter, we will present several existence theorems for second-order quasilinear elliptic equations. First, we prove the existence of finite energy solutions. The key assumptions are the structural growth of the nonlinearities appearing in the equations. We show that the induced operator has property $\alpha$ and therefore has a degree on certain subsets of its domain. Under coercivity assumption, this degree is nonzero, the operator equation is solvable, uniqueness can also be obtained when the operator is monotone.

Second, we show existence of singular solutions to several equations. Starting with cone $p$-Laplacian with and without lower order terms as model cases, we use respectively Browder and Leray-Lions theorems. Moreover, while verifying assumptions in Leray-Lions theorem, we discover that the principal part induces an operator with property $\alpha$. Therefore, equation with lower order terms yielding compact perturbations in the induced operator equation is solvable by mapping degree theory. We end the chapter with existence results for general second-order equations. The structural growth of the nonlinearities is again important. Another key assumption is the existence of a formal solution of the equation with certain behavior. The leading order and successive asymptotic terms of such formal solution in principle can be found out by formal analysis. The result on singular solutions is generalized to higher-order equations in the last chapter.

\subsection{Existence of finite energy solutions}

We investigate now second-order equations of the form

$$
-\operatorname{div} \mathcal{F}(x, \nabla u)-\operatorname{div} g_{1}(x, u)+g_{2}(x, u, \nabla u)=f(x), x \in X \backslash Y .
$$


In the present case, the basic weight $\gamma=0$, the gradient and the divergence operators are adjoint operators with respect to the Riemannian measure which is in local coordinates of the form $d g=t^{N} d t d y$.

We assume that the nonlinearities subject to the following conditions:

(A) $\mathcal{F}(x, q): X \times{ }^{c} T X \rightarrow{ }^{c} T X$ is differentiable with respect to the variables $\left(q_{1}, \ldots, q_{N}\right)$ and measurable with respect to the variable $x$. Moreover,

$$
\langle\mathcal{F}(x, q), q\rangle \geq C_{0}|q|^{p}-C_{1},
$$

and

$$
|\mathcal{F}(x, q)| \leq h_{1}(x)+|q|^{p-1}
$$

(B) $\mathcal{F}(x, q)$ is monotone with respect to the variables $q$.

(C) $g_{1}(x, u): X \times \mathbb{R} \rightarrow{ }^{c} T X$ is measurable with respect to the measure $d g$ and

$$
\left|g_{1}(x, u)\right| \leq h_{2}(x)+|u|^{m_{1}-\varepsilon}
$$

(D) $g_{2}(x, u, q): X \times \mathbb{R} \times{ }^{c} T X \rightarrow{ }^{c} T X$ fulfills

$$
\left|g_{2}(x, u, q)\right| \leq h_{3}(x)+|u|^{m_{2}-\varepsilon}+|q|^{m_{3}-\varepsilon},
$$

where $0<\varepsilon<\min \left\{m_{1}, m_{2}, m_{3}\right\}$ and

$$
\begin{aligned}
& m_{1}=\frac{p^{*}}{p^{\prime}}=\frac{(N+1)(p-1)}{N+1-p} \\
& m_{2}=p^{*}-1=\frac{(N+1)(p-1)+p}{N+1-p} \\
& m_{3}=\frac{p}{\left(p^{*}\right)^{\prime}}=\frac{(N+1)(p-1)+p}{N+1}
\end{aligned}
$$

In the assumptions (3.2) - 3.5), the product and the norm are taken in the corresponding fibers of the vector bundles, $C_{0}>0, C_{1} \geq 0$ are constants, and the functions $h_{1}, h_{2} \in$ $\mathcal{H}_{p^{\prime}}^{0,0}(X)$ and $h_{3} \in \mathcal{H}_{\left(p^{*}\right)^{\prime}}^{0,0}(X)$.

Furthermore, we assume through out this chapter that the Laplace-Beltrami operator $\Delta_{g}$ is cone-elliptic and its principal conormal symbol is invertible on the weight lines $\gamma=0$ and $\gamma+v=1$. Applying Lemma 4.25, we have

$$
D\left(\nabla_{\min }^{0, p}\right)=\mathcal{H}_{p, O}^{1,0}(X)=\mathcal{H}_{p}^{1,1}(X),(\text { see Section } 4.2 \text { for definition })
$$

under the ellipticity assumption and the weight interval $\Theta=[-1,0]$.

We will look for a weak solution of the equation (3.1) in the following sense. 
Definition 3.1. A weak solution of the equation 3.1 is a function $u \in \mathcal{H}_{p}^{1,1}(X)$ satisfying the identity follows

$$
\int_{X}\left(\mathcal{F}(x, \nabla u) \nabla v+g_{1}(x, u) \nabla v+g_{2}(x, u, \nabla u) v\right) d g=\langle f, v\rangle,
$$

on the right-hand side is the pairing of $\mathcal{H}_{p^{\prime}}^{-1,-1}(X)$ and $\mathcal{H}_{p}^{1,1}(X)$.

Taking the growth conditions of nonlinearities into account, the left-hand side is estimated by

$$
\begin{aligned}
& \left|\int_{X} \mathcal{F}(x, \nabla u) \nabla v d g\right| \leq \int_{X}\left(h_{1}(x)|\nabla v|+|\nabla u|^{p-1}|\nabla v|\right) d g \\
& \leq\left\|h_{1}\right\|_{\mathcal{H}_{p^{\prime}}^{0,0}}\||\nabla v|\|_{\mathcal{H}_{p}^{0,0}}+\|\| \nabla u\left\|_{\mathcal{H}_{p}^{0,0}}^{p-1}\right\| \nabla v \|_{\mathcal{H}_{p}^{0,0}} \\
& \left|\int_{X} g_{1}(x, u) \nabla v d g\right| \leq \int_{X}\left(h_{2}(x)+|u|^{m_{1}-\varepsilon}\right)|\nabla v| d g \\
& \leq\left\|h_{2}\right\|_{\mathcal{H}_{p^{\prime}}^{0,0}}\|\mid \nabla v\|_{\mathcal{H}_{p}^{0,0}}+\|\| \nabla v\left\|_{\mathcal{H}_{p}^{0,0}}\right\| u \|_{\mathcal{H}_{p^{\prime}\left(m_{1}-\varepsilon\right)}^{0,-}}^{m_{1}-\varepsilon} \\
& \leq\left\|h_{2}\right\|_{\mathcal{H}_{p^{\prime}}^{0,0}}\left\|\left|\nabla v\|\|_{\mathcal{H}_{p}^{0,0}}+\||| \nabla v \mid\|_{\mathcal{H}_{p}^{0,0}}\|u\|_{\mathcal{H}_{p}^{1,1}}^{m_{1}-\varepsilon}\right.\right. \\
& \left|\int_{X} g_{2}(x, u, \nabla u) v d g\right| \leq \int_{X}\left(h_{3}(x)+|u|^{m_{2}-\varepsilon}+|\nabla u|^{m_{3}-\varepsilon}\right)|v| d g \\
& \leq\left\|h_{3}\right\|_{\mathcal{H}_{p^{\prime}}^{-1,-1}}\|v\|_{\mathcal{H}_{p}^{1,1}}+\|v\|_{\mathcal{H}_{p^{*}}^{0,0}}\|u\|_{\mathcal{H}_{\left(p^{*}\right)^{\prime}\left(m_{2}-\varepsilon\right)}^{0,0}}^{m_{2}-\varepsilon}+\|v\|_{\mathcal{H}_{p^{*}}^{0,0}}\|\nabla u\|_{\mathcal{H}_{\left(p^{*}\right)^{\prime}\left(m_{3}-\varepsilon\right)}^{0,0}}^{m_{3}-\varepsilon} \\
& \leq\left\|h_{3}\right\|_{\mathcal{H}_{p^{\prime}}^{-1,-1}}\|v\|_{\mathcal{H}_{p}^{1,1}}+\|v\|_{\mathcal{H}_{p}^{1,1}}\|u\|_{\mathcal{H}_{p}^{0,0}}^{m_{2}-\varepsilon}+\|v\|_{\mathcal{H}_{p}^{1,1}}\|\nabla u\|_{\mathcal{H}_{p}^{0,0}}^{m_{3}-\varepsilon}
\end{aligned}
$$

here we have used the embeddings

$$
\begin{aligned}
& \mathcal{H}_{p}^{1,1}(X) \hookrightarrow \mathcal{H}_{m_{1} p^{\prime}}^{0,0}(X) \hookrightarrow \hookrightarrow \mathcal{H}_{p^{\prime}\left(m_{1}-\varepsilon\right)}^{0,0}(X), \\
& \mathcal{H}_{p}^{1,1}(X) \hookrightarrow \mathcal{H}_{p^{*}}^{0,0}(X) \hookrightarrow \hookrightarrow \mathcal{H}_{\left(p^{*}\right)^{\prime}\left(m_{2}-\varepsilon\right)}^{0,0}(X), \\
& \mathcal{H}_{p}^{0,0}(X)=\mathcal{H}_{\left(p^{*}\right)^{\prime} m_{3}}^{0,0}(X) \hookrightarrow \hookrightarrow \mathcal{H}_{\left(p^{*}\right)^{\prime}\left(m_{3}-\varepsilon\right)}^{0,0}(X) .
\end{aligned}
$$

Therefore, each integral on the left-hand side of (3.7) determines an operator. Let us define the operators $T, F$, and $G: \mathcal{H}_{p}^{1,1}(X) \rightarrow \mathcal{H}_{p^{\prime}}^{-1,-1}(X)$ by the formulae

$$
\begin{aligned}
\langle F u, v\rangle & =\int_{X} \mathcal{F}(x, \nabla u) \nabla v d g, \\
\left\langle G_{1} u, v\right\rangle & =\int_{X} g_{1}(x, u) \nabla v d g, \\
\left\langle G_{2} u, v\right\rangle & =\int_{X} g_{2}(x, u, \nabla u) v d g, \\
G u & :=G_{1} u+G_{2} u, T u:=F u+G u .
\end{aligned}
$$


Using the structural conditions (A), (C), (D), and applying the proposition 4.29 on $\mathrm{Ne}-$ mytskii operator, one concludes that $F, G_{1}$, and $G_{2}$ are bounded and continuous operators:

$$
\begin{aligned}
& \mathcal{F}(u(\cdot)): \mathcal{H}_{p}^{1,1}(X) \rightarrow \mathcal{H}_{p^{\prime}}^{0,0}(X), \\
& g_{1}(u(\cdot)): \mathcal{H}_{p^{\prime}\left(m_{1}-\varepsilon\right)}^{0,0}(X) \rightarrow \mathcal{H}_{p^{\prime}}^{0,0}(X), \\
& g_{2}\left(u(\cdot), q_{1}(\cdot), \ldots, q_{N}(\cdot)\right): \mathcal{H}_{\left(p^{*}\right)^{\prime}\left(m_{2}-\varepsilon\right)}^{0,0}(X) \times\left(\mathcal{H}_{\left(p^{*}\right)^{\prime}\left(m_{3}-\varepsilon\right)}^{0,0}(X)\right)^{N} \rightarrow \mathcal{H}_{\left(p^{*}\right)^{\prime}}^{0,0}(X) .
\end{aligned}
$$

Therefore, taking an arbitrary sequence $u_{n}$ converging weakly to $u$ in $\mathcal{H}_{p}^{1,1}(X)$, in particular $\nabla u_{n} \rightarrow \nabla u$ in $\left(\mathcal{H}_{p}^{0,0}(X)\right)^{N}$. Using the compact embeddings (3.9) then $u_{n} \rightarrow u$ in $\mathcal{H}_{\left(p^{*}\right)^{\prime}\left(m_{2}-\varepsilon\right)}^{0,0}(X)$ and $\mathcal{H}_{p^{\prime}\left(m_{1}-\varepsilon\right)}^{0,0}(X)$, and $\nabla u_{n} \rightarrow \nabla u$ in $\left(\mathcal{H}_{\left(p^{*}\right)^{\prime}\left(m_{3}-\varepsilon\right)}^{0,0}(X)\right)^{N}$. Continuity of the operators in 3.10 implies that $G u_{n}$ converges to $G u$ in $\mathcal{H}_{p^{\prime}}^{-1,-1}(X)$. We have checked that the mapping $G$ is a compact operator.

We will show that $F$ has property $\alpha\left(\mathcal{H}_{p}^{1,1}(X)\right)$, then $T$ as its compact perturbation also possesses this property.

Lemma 3.2. $F$ has property $\alpha\left(\mathcal{H}_{p}^{1,1}(X)\right)$ and so does $T$.

Proof. Taking an arbitrary sequence $u_{n} \rightarrow u$ in $\mathcal{H}_{p}^{1,1}(X)$ such that

$$
\limsup _{n \rightarrow \infty}\left\langle F u_{n}, u_{n}-u\right\rangle \leq 0
$$

we need to verify that $u_{n} \rightarrow u$ in $\mathcal{H}_{p}^{1,1}(X)$.

Because of the compact embedding $\mathcal{H}_{p}^{1,1}(X) \hookrightarrow \hookrightarrow \mathcal{H}_{p}^{0,0}(X)$, we obtain that $u_{n} \rightarrow u$ in $\mathcal{H}_{p}^{0,0}(X)$. Moreover, $D\left(\nabla_{\text {min }}\right)=\mathcal{H}_{p}^{1,1}(X)$, it remains to show $\nabla u_{n} \rightarrow \nabla u$ in $\left(\mathcal{H}_{p}^{0,0}(X)\right)^{N+1}$. To prove that we will check the following points:

1. the sequence $\nabla u_{n}$ converges to $\nabla u$ in measure,

2. $\lim _{\text {meas } E \rightarrow 0} \int_{E}\left|\nabla u_{n}\right|^{p} d g=0$ uniformly in $n$, where $E \subset X$.

Indeed, we denote

$$
\lambda_{n}(E)=\int_{E}\left[\mathcal{F}\left(x, \nabla u_{n}\right)-\mathcal{F}(x, \nabla u)\right]\left(\nabla u_{n}-\nabla u\right) d g .
$$

Because $\mathcal{F}$ is monotone with respect to the last $N$ components, the function under integral sign is non-negative, therefore, $0 \leq \lambda_{n}(E) \leq \lambda_{n}(X)$. Furthermore,

$$
\limsup _{n \rightarrow \infty} \lambda_{n}(X)=\limsup _{n \rightarrow \infty}\left\langle F u_{n}-F u, u_{n}-u\right\rangle \leq 0 .
$$

Hence, $\lambda_{n}(X) \rightarrow 0$ as $n \rightarrow \infty$. 
On the other hand, we can also bound $\lambda_{n}(E)$ from below by using the growth conditions (3.2), 3.3):

$$
\begin{aligned}
\lambda_{n}(E) & =\int_{E}\left[\mathcal{F}\left(x, \nabla u_{n}\right) \nabla u_{n}+\mathcal{F}(x, \nabla u)\left(\nabla u-\nabla u_{n}\right)-\mathcal{F}\left(x, \nabla u_{n}\right) \nabla u\right] d g \\
& \geq \int_{E}\left(C_{0}\left|\nabla u_{n}\right|^{p}-C_{1}\right) d g-\int_{E}\left(h_{1}(x)+|\nabla u|^{p-1}\right)\left(|\nabla u|+\left|\nabla u_{n}\right|\right) d g- \\
& -\int_{E}\left(h_{1}(x)+\left|\nabla u_{n}\right|^{p-1}\right)|\nabla u| d g
\end{aligned}
$$

Putting the negative terms to the left-hand side, we obtain that

$$
\begin{aligned}
C_{0} \int_{E}\left|\nabla u_{n}\right|^{p} d g & \leq C_{1} \mu(E)+\lambda_{n}(E)+\int_{E}\left(h_{1}(x)+|\nabla u|^{p-1}\right)\left(|\nabla u|+\left|\nabla u_{n}\right|\right) d g+ \\
& +\int_{E}\left(h_{1}(x)+\left|\nabla u_{n}\right|^{p-1}\right)|\nabla u| d g .
\end{aligned}
$$

Using Young's inequality to estimate each integral term as follows

$$
\begin{aligned}
\int_{E}\left(h_{1}(x)+|\nabla u|^{p-1}\right)\left(|\nabla u|+\left|\nabla u_{n}\right|\right) d g & \leq(1+1 / p) \int_{E}|\nabla u|^{p} d g+\frac{1}{p^{\prime}} \int_{E} h_{1}^{p^{\prime}}(x) d g \\
& +\frac{\varepsilon^{-p^{\prime}}}{p^{\prime}} \int_{E}\left(h_{1}^{p^{\prime}}(x)+|\nabla u|^{p}\right) d g+\frac{\varepsilon^{p}}{p} \int_{E}\left|\nabla u_{n}\right|^{p} d g \\
\int_{E}\left(h_{1}(x)+\left|\nabla u_{n}\right|^{p-1}\right)|\nabla u| d g & \leq \frac{1}{p^{\prime}} \int_{E} h_{1}^{p^{\prime}}(x) d g+\left(\frac{1}{p}+\frac{\varepsilon^{-p^{\prime}}}{p^{\prime}}\right) \int_{E}|\nabla u|^{p} d g \\
& +\frac{\varepsilon^{p}}{p} \int_{E}\left|\nabla u_{n}\right|^{p} d g
\end{aligned}
$$

By choosing sufficiently small $\varepsilon>0$ such that $2 \varepsilon^{p} / p<C_{0}$, we obtain

$$
\int_{E}\left|\nabla u_{n}\right|^{p} d g \leq C \int_{E}\left(h_{1}(x)^{p^{\prime}}+|\nabla u|^{p}\right) d g+\lambda_{n}(E)+C_{1} \mu(E) .
$$

It yields that $\int_{E}\left|\nabla u_{n}\right|^{p} d g \rightarrow 0$ as meas $E \rightarrow 0$ uniformly in $n$.

Now we will show that $\nabla u_{n}$ converges to $\nabla u$ in measure. For $\varepsilon, \delta>0$, set

$$
F_{\varepsilon, n}=\left\{x \in X|| \nabla u_{n}(x)-\nabla u(x) \mid \geq \varepsilon\right\}
$$

Choose two sets $E_{\delta}^{(1)}$ and $E_{\delta}^{(2)}$ such that meas $\left(E_{\delta}^{(j)}\right)<\delta / 4$, where $E_{\delta}^{(1)}$ is selected from the condition

$$
K:=\sup _{X \backslash E_{\delta}^{(1)}}\left\{|\nabla u(x)|+\left|\nabla u_{n}(x)\right|\right\}<\infty,
$$

and $E_{\delta}^{(2)}$ is selected from the condition

$$
k_{\varepsilon}:=\inf \left\{\left(\mathcal{F}(x, \xi)-\mathcal{F}\left(x, \xi^{\prime}\right)\right)\left(\xi-\xi^{\prime}\right)\right\}>0
$$


where the infimum is taken over the set

$$
\left\{x \in X \backslash E_{\delta}^{(2)},|u| \leq K,\left|\xi^{\prime}\right| \leq K ;\left|\xi-\xi^{\prime}\right| \geq \varepsilon\right\} .
$$

From the definition of the sets $E_{\delta}^{(j)}$, we have

$$
\begin{aligned}
\lambda_{n}(X) & \geq \lambda_{n}\left(F_{\varepsilon, n} \backslash\left(\cup E_{\delta}^{(j)}\right)\right) \\
& \geq k_{\varepsilon}\left(\operatorname{meas} F_{\varepsilon, n}-\operatorname{meas} E_{\delta}^{(1)}-\operatorname{meas} E_{\delta}^{(2)}\right) .
\end{aligned}
$$

Hence meas $F_{\varepsilon, n} \leq \delta / 2+\lambda_{n}(X) / k_{\varepsilon}$, and for large enough $n$, meas $F_{\varepsilon, n}<\delta$.

From these two claims and the finiteness of the measure $d g$, we obtain strong convergence of the sequence $\nabla u_{n}$ to $\nabla u$ in $\left(\mathcal{H}_{p}^{0,0}(X)\right)^{N+1}$.

We have shown that the operator $T$ satisfies the property $\alpha\left(\mathcal{H}_{p}^{1,1}(X)\right)$. We proceed by introducing further conditions that allow us to assign to $T$ a mapping degree. Our first observation requires strict growth condition.

Theorem 3.3. Suppose that the nonlinearities subject to the conditions (3.2), (3.3), (3.4), (3.5), and monotone condition (B). Furthermore, assume that for almost all $(x, u, q) \in$ $X \times \mathbb{R} \times{ }^{c} T X$, it holds

$$
\mathcal{F}(x, q) q+g_{1}(x, u) q+g_{2}(x, u, q) u \geq C_{2}|q|^{p}+C_{3}|u|^{p}-C_{4} .
$$

Given $f \in \mathcal{H}_{p^{\prime}}^{-1,-1}(X)$, there exists a solution $u \in \mathcal{H}_{p}^{1,1}(X)$ to 3.1 .

Proof. Now, we use the condition 3.11 to verify the coercivity of $T$. Indeed, take an arbitrary element $u \in \mathcal{H}_{p}^{1,1}(X)$, we obtain

$$
\begin{aligned}
\langle T u, u\rangle & =\int_{X}\left[\mathcal{F}(x, \nabla u) \nabla u+g_{1}(x, u) \nabla u+g_{2}(x, u, \nabla u) u\right] d g \\
& \geq C_{2} \int_{X}|\nabla u|^{p} d g+C_{3} \int_{X}|u|^{p} d g-C_{4} \\
& \geq C\|u\|_{\mathcal{H}_{p}^{1,1}}^{p}-C_{4} .
\end{aligned}
$$

Therefore,

$$
\lim _{\|u\| \rightarrow \infty} \frac{\langle T u, u\rangle}{\|u\|_{\mathcal{H}_{p}^{1,1}}}=\infty .
$$

Because of this limit, there exists a value $R \in \mathbb{R}_{+}$such that for all $\|u\|>R$ the following inequality holds

$$
\frac{\langle T u, u\rangle}{\|u\|_{\mathcal{H}_{p}^{1,1}}} \geq\|f\|_{\mathcal{H}_{p^{\prime}}^{-1,-1}}+1 .
$$


Therefore, $f$ does not belong to the image $T(\partial B(0, R))$, so the degree of $T$ on the set $\overline{B(0, R)}$ with respect to $f$ is well-defined. Moreover, for all $u \in \partial B(0, R)$

$$
\langle T u, u\rangle \geq R\left(\|f\|_{\mathcal{H}_{p^{\prime}}^{-1,-1}}+1\right)>0,
$$

which implies that $\operatorname{Deg}(T, \overline{B(0, R)}, f)=1$. Applying theorem 2.30, there exists a solution $u \in \mathcal{H}_{p}^{1,1}(X)$ to the equation $T u=f \in \mathcal{H}_{p^{\prime}}^{-1,-1}(X)$.

Remark 3.4. We aim at using the mapping degree to prove existence results, therefore, the approach is first to assume some hypotheses that make the procedure works, and then to determine which kind of nonlinearities are solvable by this scheme. However, due to more requirements on the nonlinearities, the range of application is narrow, for example, the $p$-Laplace equation does not satisfy the growth condition (3.11). We would like to relax this condition.

Theorem 3.5. Suppose that the cone Laplacian $\Delta_{g}$ is elliptic with respect to the weight line $\mathfrak{R} z=1$ and the nonlinearities subject to the conditions (3.2), (3.3), (3.4), (3.5). Furthermore, assume that for almost all $(x, u, q) \in X \times \mathbb{R} \times{ }^{c} T X$, it holds

$$
\mathcal{F}(x, q) q+g_{1}(x, u) q+g_{2}(x, u, q) u \geq C_{2}|q|^{p}-C_{4} .
$$

Given $f \in \mathcal{H}_{p^{\prime}}^{-1,-1}(X)$ which is perpendicular to $\operatorname{Ker} \nabla$, there exists a solution $u \in \mathcal{H}_{p}^{1,1}(X)$ to 3.1 .

Let us recall a result in functional analysis concerning the dual space of a quotient space of a Banach space. This result will give us necessary, and later turn to be sufficient, conditions on the right-hand side for the equation to be solvable.

Let $X$ be a Banach space and $M$ be a closed linear subspace of $X$. The quotient $V=X / M$ is itself a Banach space with the norm induced from the norm of $X$, namely, for $\bar{v} \in V$,

$$
\|v\|_{V}=\inf _{u \in M}\|v+u\| .
$$

The annihilator of $M$ in the dual space $X^{\prime}$ of $X$ is the subspace

$$
M^{\perp}=\left\{f \in V^{\prime}: M \subset \operatorname{Ker} f\right\} .
$$

Then the dual space of the quotient $X / M$ can be identified with $M^{\perp}, V^{*} \cong M^{\perp}$, and the dual space of $M$ can be identified with the quotient $X^{\prime} / M^{\perp}, M^{*} \cong X^{\prime} / M^{\perp}$. Therefore, the constraint that makes an element $f \in X^{\prime}$ belong indeed to $M^{*}$ is that $f$ is perpendicular to 
$M$. Moreover, in case $M$ has finite dimension, then it has a complement in $X$, namely $V$, which is isomorphic to $X / M$.

In Theorem 3.5, the constraint that $f \in \mathcal{H}_{p^{\prime}}^{-1,-1}(X)$ perpendicular to Ker $\nabla$ means that $f$ belongs to the dual subspace $M^{*}$ of some subspace $M$ of $\mathcal{H}_{p}^{1,1}(X)$ which is a complement of $\operatorname{Ker} \nabla$ in $\mathcal{H}_{p}^{1,1}(X)$. We will prove by defining an operator $\widetilde{T}:=\left.T\right|_{M}: M \rightarrow \mathcal{H}_{p^{\prime}}^{-1,-1}(X)$ and applying mapping degree theory to this restricted operator.

Proof. Applying Corollary 4.27 to the Laplacian, one knows that $\operatorname{Ker} \nabla \cap \mathcal{H}_{p}^{1,1}(X)$ has finite dimension. We call $M$ the topological complement of $\operatorname{Ker} \nabla$ in $\mathcal{H}_{p}^{1,1}(X)$. Applying again Corollary (4.27) to the cone gradient $\nabla$, we conclude that for all $u \in M$, a Poincarétype inequality holds and consequently,

$$
C_{1}\|u\|_{\mathcal{H}_{p}^{1,1}} \leq\|\| \nabla u\left\|_{\mathcal{H}_{p}^{0,0}} \leq C_{2}\right\| u \|_{\mathcal{H}_{p}^{1,1}}
$$

Hence, it holds for all $u \in M$ that

$$
\frac{\langle T u, u\rangle}{\|u\|_{\mathcal{H}_{p}^{1,1}}} \geq C\|\| \nabla u \|_{\mathcal{H}_{p}^{0,0}}^{p-1}-\frac{C_{4}}{\|u\|_{\mathcal{H}_{p}^{1,1}}}
$$

We obtain the weak coercivity

$$
\lim _{\substack{u \in M \\\|\nabla u\| \rightarrow \infty}} \frac{\langle T u, u\rangle}{\|u\|_{\mathcal{H}_{p}^{1,1}}}=\infty .
$$

Restricting the operator $T$ to $M$, then the operator $\widetilde{T}:=\left.T\right|_{M}: M \rightarrow M^{*}$ inherits continuity and the property $\alpha$. Moreover, $\widetilde{T}$ is coercive. Consequently, for a given $f \in M^{*}$, there exists an $R \in \mathbb{R}_{+}$such that for all $u \in M$ and $\|\nabla u\| \|>R$ the following inequality holds

$$
\frac{\langle T u, u\rangle}{\|u\|_{\mathcal{H}_{p}^{1,1}}} \geq\|f\|_{\mathcal{H}_{p^{\prime}}^{-1,-1}}+1 .
$$

It implies that $f$ does not belong to the image $\widetilde{T}(\partial D)$, and we can assign to $\widetilde{T}$ the degree $\operatorname{Deg}(\widetilde{T}, \overline{B(0, R)}, f)$. Moreover, for all $u \in \partial D$

$$
\langle T u, u\rangle \geq C\left(\|f\|_{\mathcal{H}_{p^{\prime}}^{-1,-1}}+1\right)>0
$$

which implies that $\operatorname{Deg}(T, \bar{D}, f)=1$. The existence of a solution $u \in M \subset \mathcal{H}_{p}^{1,1}(X)$ to the equation $T u=f \in M^{*}$ is obvious by Theorem 2.30 .

Remark 3.6. We have seen that the nonlinearities (3.6) in our assumptions are as general as in the non-degenerate case ([10, Section 7.6A], [38, Chapter 1]). What counts is the adapted embeddings (3.9). We will see that in the case of singular solutions, the nonlinearities are slightly narrower. 
Remark 3.7. If we consider smaller growth of the nonlinearities, then the coercivity on the subset $D$ can be shown without further assumption (3.11). More precisely, if we require

$$
\widetilde{m}_{1}, \widetilde{m}_{2}, \widetilde{m}_{3} \leq p-1
$$

and make slight changes in the estimate (3.8) in terms of the norms $\|u\|_{\mathcal{H}_{p}^{1,1}},\|\| \nabla u \|_{\mathcal{H}_{p}^{0,0}}$. Then we obtain straightforward

$$
\frac{\langle T u, u\rangle}{\|u\|_{\mathcal{H}_{p}^{1,1}}} \geq C_{0}\|\| \nabla u\left\|_{\mathcal{H}_{p}^{0,0}}^{p-1}-C\right\|\|\nabla u\|_{\mathcal{H}_{p}^{0,0}}^{p-1-\varepsilon}, \varepsilon>0 .
$$

Therefore, coercivity holds

$$
\lim _{\|u\| \rightarrow \infty} \frac{\langle T u, u\rangle}{\|u\|_{\mathcal{H}_{p}^{1,1}}}=\infty .
$$

Existence can be obtained as in theorem 3.5 for all $f \in M^{*}$.

Remark 3.8. We can repeat verbatim the proofs above to the case of general operators $A$, $A^{\prime}$. The point is that the ellipticity of $A^{\prime} A$ yields that $D\left(A_{\min }\right)$ is compactly embedded into $\mathcal{H}_{p}^{0,0}(X)$, which is essential when verifying the property $(S)_{+}$of the induced operator. The weak coercivity holds consequently as a Poincaré-type inequality holds.

\subsection{Singular solutions to equations involving $p$-Laplacian}

We confine ourselves in this part to the question about existence of singular solutions to equations involving the cone $p$-Laplace operator. This operator is a prototype of quasilinear elliptic operators whose properties motivate us the choice of the class of nonlinearities under observation. As we have mentioned in the introduction, we will consider $p<N+1$. Moreover, we restrict ourselves to the case $p \geq 2$, as we will need some specific inequalities for vectors in this case.

Concerning the question about existence of singular solutions with infinite energy, there have been several results for quasilinear elliptic equations. We would like to mention the works of Kichenassamy-Véron [21] on the $p$-Laplacian

$$
-\Delta_{p} u=\delta
$$

and of Friedman-Véron [12] on the $p$-Laplacian with an absorption term

$$
-\Delta_{p} u+|u|^{q-1} u=\delta, p-1 \leq q<\frac{(N+1)(p-1)}{N+1-p},
$$


in a subset $\Omega$ of the Euclidean space $\mathbb{R}^{N+1}$, in which the authors employed approximation scheme and comparison principle. However, this approach does not work for higher-order equations. More details, these authors showed the existence of a solution which behaves like the singular $p$-harmonic function

$$
\mu_{p}(x)=C t^{-\alpha}, \alpha=\frac{N+1-p}{p-1}>0
$$

when approaching the boundary. We classify this equation later as Serrin's case, meaning that the nonlinear growth of lower order terms is dominated by the nonlinear growth of the highest order term. For equations with dominant growth of lower order terms, the exponent $-\alpha$ is called a weak singularity. There might exist a solution with a strong singularity, namely $u(x) \sim|x|^{-p /(q-p+1)}$ as $|x| \rightarrow 0$. For example, in the semilinear case, the equation $-\Delta u+|u|^{q-1} u=0$ on a ball in $\mathbb{R}^{N+1}$ with appropriate boundary condition has one solution $x \mapsto l_{q, N}|x|^{-2 /(q-1)}$. However, in the quasilinear case, the existence of such a strongly singular solution has not yet been known. We are only certain that the coefficient of the leading term is unique (see [41, Theorem 5.7]):

$$
\lambda_{N, p, q}=\left(\left(\frac{p}{q-p+1}\right)^{p-1}\left(\frac{p q}{q-p+1}-(n+1)\right)\right)^{\frac{1}{q-p+1)}}
$$

Isolated singularities for the $p$-Laplacian with a source term were classified in [41, Section 5.3], however without any existence results.

Let me recall the well-known existence results concerning singular solutions. There have been several approaches to deal with the existence of blow-up solutions. One approach is to use a truncation method and to renormalize the solution in each finite interval of the range. To my knowledge the best result in this direction is by Dal Maso, Murat, Orsina, and Prignet ([7]) where the right-hand side is general measure data. However, this method cannot be applied to higher-order equations, and in their work, there was also no information about behavior of solutions. Another approach is to use approximation method, where the convergence in certain sense of the approximating solutions and the desired behavior of solutions are checked by using comparison principle, we refer to the papers [12, 21].

Our approach uses monotonicity and topological methods: first, we will use Browder theorem to study the singular $p$-Laplacian, second, we will use Leray-Lions theorem to study equations involving lower order terms. Following these theorems, one requires a coercivity assumption. We are in the case that the principal part induces a monotone 
operator and dominates the lower order part, and we look for solutions with weak singularities.

One essential assumption is the existence of a formal solution of the observed equation. A formal solution of quasilinear equations will satisfy it to infinite order, therefore, by formal asymptotic analysis, leading order and successively further asymptotic terms can be worked out. After finite steps, one can assume to have reached some space of certain smoothness containing the remainder part. In this function space, later denoted by $\mathfrak{X}$, we control the remainder by two norms, one comes from the singular part. Due to estimate concerning this norm, we restrict ourselves to the case $2 \leq p<N+1$.

Provided the existence of formal solutions, one expects to use inverse function theorem to obtain local existence. Using nonlinear functional analysis, we obtain global results. And we will see in the next chapter, our approach using the topological method can be applied to higher-order equations.

\subsubsection{Singular solutions of cone $p$-Laplacian}

Consider the equation

$$
\left\{\begin{array}{l}
-\operatorname{div}\left(|\nabla u|^{p-2} \nabla u\right)=f(x), x \in X \backslash Y, \\
u \sim c t^{-\alpha} \text { as } x \rightarrow Y .
\end{array}\right.
$$

We will prove that there is also a formal solution of (3.14) that demonstrates the same exponent as in this non-flat metric case.

Lemma 3.9. There is a formal solution $e(t, y) \sim c t^{-\alpha}, c \in \mathbb{R}$, where $\alpha>0$ is Serrin's exponent.

Proof. Take a section $u$, then the gradient

$$
\nabla u=\left(\frac{\partial u}{\partial t}, t^{-1} \frac{\partial u}{\partial y^{1}}, \ldots, t^{-1} \frac{\partial u}{\partial y^{N}}\right)
$$

with respect to the basis $\frac{\partial}{\partial t}, t^{-1} \frac{\partial}{\partial y^{1}}, \ldots, t^{-1} \frac{\partial}{\partial y^{N}}$ of unit vectors of the cone tangent bundle. Therefore, the principal conormal symbol is

$$
\sigma_{M}^{1}(\nabla)(z)=\left(z, \nabla_{Y}(0)\right) \in \operatorname{Diff}^{1}\left(Y, X \times\left.\mathbb{R}\right|_{Y},\left.{ }^{c} T X\right|_{Y}\right)
$$

We look for a solution $u$ with the leading behaviour $u(t, y) \sim t^{-\alpha} \varphi(y)$. Then

$$
\nabla u \sim t^{-\alpha-1}\left(\alpha \varphi(y), \nabla_{g_{Y}(0)} \varphi(y)\right) .
$$


Moreover, $|\nabla u|=t^{-\alpha-1} \sqrt{\alpha^{2} \varphi^{2}+\left|\nabla_{g_{Y}(0)} \varphi\right|^{2}}=: \Phi(y)^{1 /(p-2)}$. Therefore,

$$
|\nabla u|^{p-2} \nabla u \sim t^{-(p-1)(\alpha+1)} \Phi(y)\left(\alpha \varphi(y), \nabla_{g_{Y}(0)} \varphi(y)\right)
$$

Generally, given a vector $\hat{X}=\left(X^{0}, X^{1}, \ldots, X^{N}\right)=\left(X^{0}, X\right)$ expressed in local coordinates $t, y_{1}, \ldots, y_{N}$ then

$$
\operatorname{div} \hat{X}=\frac{1}{t^{N}|G|}\left(\frac{\partial}{\partial t}\left(t^{N}|G| X^{0}\right)+\sum_{j=1}^{N} \frac{\partial}{\partial y_{j}}\left(t^{N}|G| X^{j}\right)\right)
$$

We already know that

$$
\sigma_{M}^{v}\left(A^{*}\right)(z)=\sigma_{M}^{v}(N+1-2 \gamma-\bar{z}-v)^{*},
$$

where the latter star means the adjoint operator on $Y$ with respect to the measure $d y$. Hence,

$$
\sigma_{M}^{1}(\operatorname{div})(z)=\sigma_{M}^{1}(\nabla)(N-\bar{z})^{*}
$$

As $\sigma_{M}^{1}(\nabla)(z)=\left(z, \nabla_{Y(0)}\right)$, we have

$$
\sigma_{M}^{1}(\operatorname{div})(z)=\bar{z} X^{0}+\nabla_{g_{Y}(0)} \cdot X
$$

Therefore, after applying the divergence operator, we obtain

$$
\nabla \cdot\left(|\nabla u|^{p-2} \nabla u \sim t^{-(p-1)(\alpha+1)-1}\left\{(N-\overline{(p-1)(\alpha+1)}) \alpha \Phi(y) \varphi(y)+\nabla_{g_{Y}(0)} \cdot\left(\Phi(y) \nabla_{g_{Y}(0)} \varphi(y)\right)\right\}\right.
$$

The leading behavior of the formal solution will necessarily satisfy the equation

$$
\left\{(N-(p-1)(\alpha+1)) \alpha \Phi(y) \varphi(y)+\nabla_{g_{Y}(0)} \cdot\left(\Phi(y) \nabla_{g_{Y}(0)} \varphi(y)\right)\right\}=0 .
$$

A sufficient condition is

$$
\begin{aligned}
& (N-(p-1)(\alpha+1)) \alpha \Phi(y) \varphi(y)=0 \\
& \left.\nabla_{g_{Y}(0)} \cdot\left(\Phi(y) \nabla_{g_{Y}(0)} \varphi(y)\right)\right)=0 .
\end{aligned}
$$

We easily see that a special case is when $N-(p-1)(\alpha+1)=0$, or $\alpha=\frac{N+1-p}{p-1}$, and $\varphi(y)=$ const.

As mentioned above, we will use Browder and Leray-Lions theorems to obtain the existence for the equation (3.14) and equations with lower order terms. Among the requirement of these theorems is the coercivity. We have experienced in Theorem 3.5 that one cannot freely set the coercivity assumption. However, this property can be shown 
for some restriction of the induced operator. We would expect to work again with a subspace in which a Poincaré-type inequality holds. On the other hand, we are working with solutions whose energy blows up, so integration by part no longer works, the induced operator can only be estimated by evaluating at test functions. Therefore, we will introduce a function space containing $C_{c}^{\infty}(X \backslash Y)$ as a dense subspace, and a reduction to an operator equation based on the fact that one can find $e$ such that $\Delta_{p} e$ vanishes to sufficiently large order.

Let us define $\mathfrak{X}=\mathcal{H}^{1, \gamma}(X ; E) \cap \mathcal{H}_{p}^{1,1}(X ; E)$, where $\gamma=1+\frac{N(p-2)}{2(p-1)} . C_{c}^{\infty}(X \backslash Y)$ is dense in $\mathfrak{X}$, and $\mathfrak{X}$ is reflexive. Denote by $\mathfrak{X}^{*}$ its dual space, which is

$$
\mathfrak{X}^{*}=\mathcal{H}^{-1,-\gamma}(X ; E)+\mathcal{H}_{p^{\prime}}^{-1,-1}(X ; E), p^{\prime}=\frac{p}{p-1} .
$$

(recall that we have identified $E$ and $E^{\prime}$ via the bundle metric $h_{E}$ ).

We require later that the right-hand side $f \in \mathfrak{X}^{*}$ satisfying further that $f$ is perpendicular to $\operatorname{Ker} \nabla \cap \mathfrak{X}$, which actually means that $f \in \mathfrak{B}^{*}$, where $\mathfrak{B}$ is a topological complement of $\operatorname{Ker} \nabla$ in $\mathfrak{X}$. In this subspace, we can use the equivalent norm given by the sum of two seminorms in $\mathcal{H}^{1, \gamma}(X)$ and $\mathcal{H}_{p}^{1,1}(X)$, namely

$$
\|v\|_{V} \sim\|v\|_{1}+\|v\|_{2}
$$

where

$$
\|v\|_{1}=\left(\int_{X} t^{2-2 \gamma}|\nabla v|^{2} d g\right)^{\frac{1}{2}},\|v\|_{2}=\|\nabla v\|_{L^{p}(d g)},
$$

Because of the assumption of $e$, the first seminorm $\|v\|_{1}$ actually is equivalent to

$$
\|v\|_{1}=\left(\int_{X} t^{2-2 \gamma}|\nabla v|^{2} d g\right)^{\frac{1}{2}} \sim\left(\int_{X}|\nabla e|^{p-2}|\nabla v|^{2} d g\right)^{\frac{1}{2}}
$$

We obtain the following existence theorem:

Theorem 3.10. Let $p \geq 2$ and assume that the equation (3.14) has an approximate solution $e(x) \sim C t^{-\alpha}$ such that $\Delta_{p} e \in \mathfrak{B}^{*}$. Given an $f \in \mathfrak{X}^{*}$, such that $f$ is perpendicular to $\operatorname{Ker} \nabla$ in $\mathfrak{X}$, there exists a solution to (3.14) which is of the form $e+v, v \in \mathfrak{X}$. The part $v$ is unique modulo $\operatorname{Ker} \nabla$.

Proof. Let us define an operator $T: \mathfrak{X} \rightarrow \mathfrak{X}^{*}$ by

$$
T v=-\operatorname{div}\left(|\nabla(e+v)|^{p-2} \nabla(e+v)\right)+\operatorname{div}\left(|\nabla e|^{p-2} \nabla e\right) .
$$


The operator $T$ is well-defined. Indeed, for an arbitrary function $v \in \mathfrak{X}$ we show that $T v \in \mathfrak{X}^{*}$ by taking a test function $w \in C_{c}^{\infty}(X \backslash Y)$ and estimating

$$
\begin{aligned}
|\langle T v, w\rangle| & =\left|\int_{X}\left[\left(|\nabla(e+v)|^{p-2} \nabla(e+v)-|\nabla e|^{p-2} \nabla e\right) \nabla w\right] d g\right| \\
& \leq(p-1) \int_{X}|\nabla v \| \nabla w| \int_{0}^{1}|\nabla e+s \nabla v|^{p-2} d s d g, \text { use (2.12) } \\
& \leq C \int_{X}\left(|\nabla e|^{p-2}+|\nabla v|^{p-2}\right)|\nabla v \| \nabla w| d g, \text { use (2.14) } \\
& \leq C\left(\int_{X}|\nabla e|^{p-2}|\nabla v|^{2} d g\right)^{\frac{1}{2}}\left(\int_{X}|\nabla e|^{p-2}|\nabla w|^{2} d g\right)^{\frac{1}{2}}+C\left|\|\nabla v \mid\|_{L^{p}}^{p-1}\|\| \nabla w \|_{L^{p}}\right. \\
& \leq C\left(\|v\|_{\mathfrak{X}}\right)\|w\|_{\mathfrak{X}} .
\end{aligned}
$$

As $C_{c}^{\infty}(X \backslash Y)$ is dense in $\mathfrak{X}$, it holds also for all $w \in \mathfrak{X}$,

$$
|\langle T v, w\rangle| \leq C\left(\|v\| \|_{\mathfrak{X}}\right)\|w\|_{\mathfrak{X}} .
$$

So $T$ is well-defined. We need to check the following points.

(i) $T$ is bounded. This property is obvious by using the estimate 3.16 , it holds for $v \in \mathfrak{X}$

$$
\|T v\|_{\mathfrak{F}^{*}} \leq C\|v\|_{\mathfrak{x}}
$$

(ii) $T$ is demi-continuous as it is actually continuous. Indeed, given an arbitrary sequence $\left\{v_{n}\right\}$ converging to $v$ in $\mathfrak{X}$, we repeat the estimate above for $v_{n}$ and $v$, and verify for an arbitrary $w \in C_{c}^{\infty}(X \backslash Y)$ that

$$
\begin{aligned}
\left|\left\langle T v_{n}-T v, w\right\rangle\right| & =\mid \int_{X}\left[\left(\left|\nabla\left(e+v_{n}\right)\right|^{p-2} \nabla\left(e+v_{n}\right)-\left(|\nabla(e+v)|^{p-2} \nabla(e+v)\right) \nabla w\right] d g \mid\right. \\
& \leq(p-1) \int_{X}|\nabla v||\nabla w| \int_{0}^{1}\left|\nabla e+\nabla v+s\left(\nabla v_{n}-\nabla v\right)\right|^{p-2} d s d g \\
& \leq C \int_{X}\left(|\nabla e|^{p-2}+|\nabla v|^{p-2}+\left|\nabla\left(v_{n}-v\right)\right|^{p-2}\right)\left|\nabla\left(v_{n}-v\right) \| \nabla w\right| d g \\
& \leq C\left(\left\|v_{n}-v\right\|_{1}\|w\|_{1}+\left\|\nabla\left(v_{n}-v\right)\right\|_{2}^{p-2}\|\nabla v\|_{2}\|\nabla w\|_{2}\right)
\end{aligned}
$$

So the left-hand side $\left\langle T v_{n}-T v, w\right\rangle \rightarrow 0$ when $n \rightarrow \infty$ for all $w \in C_{c}^{\infty}(X \backslash Y)$, this convergence also holds for all $w \in \mathfrak{X}$.

All the properties above can be passed to the properties of the restriction $\widetilde{T}: \mathfrak{B} \rightarrow$ $X^{*}$ of $T$ to the subspace $\mathfrak{B}$ with the induced topology. 
(iii) Moreover, $\widetilde{T}$ is a coercive operator. First, testing with a function $v \in C_{c}^{\infty}(X \backslash Y)$, we have

$$
\begin{aligned}
\langle T v, v\rangle & =\int_{X}\left(|\nabla(e+v)|^{p-2} \nabla(e+v)-|\nabla e|^{p-2} \nabla e\right) \nabla v d g \\
& \geq \int_{X} 2^{-1}|\nabla v|^{2}\left(|\nabla e|^{p-2}+|\nabla(e+v)|^{p-2}\right) d g,(\text { use }(2.10)) \\
& \geq \frac{1}{4} \int_{X}|\nabla v|^{2}\left(|\nabla e|^{p-2}+\left(|\nabla e|^{p-2}+|\nabla(e+v)|^{p-2}\right)\right) d g \\
& \geq \frac{1}{4} \int_{X}|\nabla v|^{2}\left(|\nabla e|^{p-2}+2^{3-p}|\nabla v|^{p-2}\right) d g, \text { (use }[2.10) \\
& \geq C \int_{X}\left(|\nabla v|^{2}|\nabla e|^{p-2}+|\nabla v|^{p}\right) d g
\end{aligned}
$$

Therefore, for all $v \in \mathfrak{X}$, this estimate also holds. Restricting to $v \in \mathfrak{B}$, passing to the operator $\widetilde{T}$, and using the equivalence of norms (3.15), we obtain

$$
\frac{\langle\widetilde{T} v, v\rangle}{\|v\|_{\mathfrak{X}}} \geq \frac{C\left(\|v\|_{1}^{2}+\|v\|_{2}^{p}\right)}{\|v\|_{1}+\|v\|_{2}} \rightarrow \infty
$$

as $\|v\|_{\mathfrak{X}}=\|v\|_{1}+\|v\|_{2} \rightarrow \infty$. Hence, $\widetilde{T}$ is coercive in $\mathfrak{B}$.

(iv) $T$ is monotone, and so is $\widetilde{T}$. Indeed, taking two arbitrary elements $u, v \in \mathfrak{X}$ and two sequences $\left\{u_{n}\right\},\left\{v_{m}\right\} \subset C_{c}^{\infty}(X \backslash Y)$ converging to $u, v$ respectively, whose elements have compact supports. We have for $u_{n}, v_{m}$ :

$$
\begin{aligned}
\left\langle T u_{n}-T v_{m}, u_{n}-v_{m}\right\rangle= & \int_{X} \nabla\left(u_{n}-v_{m}\right)\left(\left|\nabla\left(e+u_{n}\right)\right|^{p-2} \nabla\left(e+u_{n}\right)-\right. \\
& \left.\left.-\left|\nabla\left(e+v_{m}\right)\right|^{p-2} \nabla\left(e+v_{m}\right)\right) d g \geq 0, \text { (use 2.13) }\right)
\end{aligned}
$$

Actually, this integral is taken over $\operatorname{supp} u_{n} \cap \operatorname{supp} v_{m}$ and one can use integration by part. Moreover, one has

$$
\begin{aligned}
\left\langle T u_{n}-T v_{m}, u_{n}-v_{m}\right\rangle=\langle T u-T v, u-v\rangle+\left\langle T u_{n}-T u, u-v\right\rangle \\
+\left\langle T u_{n}-T v_{m},\left(u_{n}-u\right)+\left(v-v_{m}\right)\right\rangle-\left\langle T v_{m}-T v, u-v\right\rangle
\end{aligned}
$$

Because $u_{n} \rightarrow u, v_{m} \rightarrow v$, and $T$ is continuous, we have $\left\{T u_{n}\right\}$ and $\left\{T v_{m}\right\}$ are bounded sequences which converge to $T u$ and $T v$ respectively. Therefore, the last three terms in the equality above converge to 0 . Finally, we obtain

$$
\langle T u-T v, u-v\rangle=\lim _{m, n \rightarrow \infty}\left\langle T u_{n}-T v_{m}, u_{n}-v_{m}\right\rangle \geq 0 .
$$

Passing again to $\widetilde{T}$, it yields that $\widetilde{T}$ is monotone in $\mathfrak{B}$. 
Applying Browder's theorem, $\widetilde{T}$ is surjective, therefore the equation $T u=f \in \mathfrak{B}^{*}$ has a solution in $\mathfrak{B}$. We have assumed that $\Delta_{p} e \in \mathfrak{B}^{*}$. It implies that the equation

$$
-\Delta_{p}(e+v)=f-\Delta_{p} e \in \mathfrak{B}^{*}
$$

has at least one solution $v$ for all right-hand side belonging to $\mathfrak{B}^{*}$. It completes the proof of existence.

Moreover, this solution is unique. Assume the contrary that $v, w \in \mathfrak{X}$ are two solutions to the equation. There are two sequences $\left\{u_{n}\right\}$ and $\left\{v_{m}\right\}$ belonging to $C_{c}^{\infty}(X \backslash Y)$ which approximate $u$ and $v$ respectively. That $T$ is a continuous operator implies

$$
T u_{n}-T v_{m} \rightarrow T u-T v \text { in } \mathfrak{X}^{*} \text { as } m, n \rightarrow \infty \text {. }
$$

Together with the convergence $u_{n}-v_{m} \rightarrow u-v$ in $\mathfrak{X}$, we have

$$
\left\langle T u_{n}-T v_{m}, u_{n}-v_{m}\right\rangle \rightarrow\langle T u-T v, u-v\rangle=0 .
$$

as $m, n \rightarrow \infty$.

On the other hand, we already know from (2.17)that

$$
0 \leq C \int_{X}\left|\nabla u_{n}-\nabla v_{m}\right|^{p} d g \leq\left\langle T u_{n}-T v_{m}, u_{n}-v_{m}\right\rangle .
$$

Passing to the limit as $m, n \rightarrow \infty$, it yields

$$
\lim _{m, n \rightarrow \infty} \int_{X}\left|\nabla u_{n}-\nabla v_{m}\right|^{p} d g=0,
$$

which actually gives us

$$
\int_{X}|\nabla u-\nabla v|^{p} d g=0 .
$$

Because $X$ is connected, we conclude that $u-v \equiv C$ almost everywhere.

\subsubsection{Singular solutions of the $p$-Laplacian with an absorption term}

We proceed with an equation having lower order terms which is given by

$$
-\operatorname{div}\left(|\nabla u|^{p-2} \nabla u\right)+g(u)=f(x), x \in X \backslash Y .
$$

This equation is said to involve an absorption term when $g(u)$ is an increasing function. An example is $g(u)=|u|^{q-1} u, q>1$. Existence of a solution with weak singularity, namely $u(x) \sim c t^{-\alpha}$, where $\alpha$ is as before Serrin's exponent, has been shown by Friedman-Véron in Euclidean space setting when $p-1<q<\frac{(N+1)(p-1)}{N+1-p}$. Therefore, we expect that a similar result works for the equation (3.17), namely, we can look for a solution whose regular part stays in the space $\mathfrak{X}$ or $\mathfrak{B}$. 
Theorem 3.11. Let us suppose all assumptions as in Theorem 3.10. Assume further that the nonlinearity $g$ is an increasing function and satisfies

$$
0 \leq g^{\prime}(s) \leq C|s|^{p-2}, \text { for all } s \in \mathbb{R} \text {. }
$$

Given an $f \in \mathfrak{B}^{*}$, there exists a solution to (3.17) which is of the form $e+v$, where $e \sim C t^{-\alpha}$ is the singular part uniquely determined and $v \in \mathfrak{X}$.

Proof. Let us define the operators $T, F$, and $G: \mathfrak{X} \rightarrow \mathfrak{X}^{*}$ by

$$
\begin{aligned}
& F v=-\operatorname{div}\left(|\nabla(e+v)|^{p-2} \nabla(e+v)\right)+\operatorname{div}\left(|\nabla e|^{p-2} \nabla e\right), \\
& G v=g(e+v)-g(e), \\
& H v=(F+G) v .
\end{aligned}
$$

We already know that $F: \mathfrak{X} \rightarrow \mathfrak{X}^{*}$ is a bounded, continuous, and coercive operator. We prove similarly the continuity of $G$. Moreover, $G$ is compact.

Indeed, taking $w \in C_{c}^{\infty}(X \backslash Y), v \in \mathfrak{X}$, and estimating

$$
\begin{aligned}
|\langle G v, w\rangle| & =\left|\int_{X}(g(e+v)-g(e)) w d g\right| \leq C \int_{X}\left|g^{\prime}(e+\lambda(x) v)\right||v w| d g, \text { where } 0 \leq \lambda(x) \leq 1, \\
& \leq C \int_{X}\left(|e|^{p-2}+|v|^{p-2}\right)|v w| d g \leq C\left(\|v\|_{\mathcal{H}^{0, \delta}}\|w\|_{\mathcal{H}^{0, \delta}}+\|v\|_{\mathcal{H}_{p}^{0,0}}^{p-1}\|w\|_{\mathcal{H}_{p}^{0,0}}\right) \\
& \leq C\left(\|v\|_{\mathfrak{X}}\right)\|w\|_{\mathfrak{X}},
\end{aligned}
$$

here $\delta=\frac{(N+1-p)(p-2)}{2(p-1)}$.

Because $C_{c}^{\infty}(X \backslash Y)$ is dense in $\mathfrak{X}$, the inequality above holds also for $w \in \mathfrak{X}$. Therefore, $G$ is well-defined. Furthermore, it is bounded as we have

$$
\|G u\|_{\mathfrak{X}^{*}} \leq C\|v\|_{\mathfrak{X}} .
$$

The continuity of $G$ can be proved similarly to the proof of Theorem 3.10 .

Taking an arbitrary weakly convergent sequence $\left\{v_{n}\right\}, v_{n} \rightarrow v$ in $\mathfrak{X}$. Because of the compact embeddings

$$
\mathcal{H}_{p}^{1,1}(X) \hookrightarrow \hookrightarrow \mathcal{H}_{p}^{0,0}(X), \quad \mathcal{H}^{1, \gamma}(X) \hookrightarrow \hookrightarrow \mathcal{H}^{0, \delta}(X),
$$

as $\delta<\gamma$, we have $v_{n} \rightarrow v$ in $\mathcal{H}_{p}^{0,0}(X) \cap \mathcal{H}^{0, \gamma-1}(X)$. Hence,

$$
\begin{aligned}
\left\|G v_{n}-G v\right\|_{X^{*}} & =\sup _{\|w\|_{\mathfrak{x}}=1}\left|\left\langle G v_{n}-G v, w\right\rangle\right|=\sup _{\|w\|_{\mathfrak{X}}=1}\left|\int_{X}\left(g\left(e+v_{n}\right)-g(e+v)\right) w d g\right| \\
& \leq C \sup _{\|w\|_{\mathfrak{x}}=1} \int_{X}\left(|e|^{p-2}+\left|v_{n}-v\right|^{p-2}\right)\left|v_{n}-v \| w\right| d g \\
& \leq C\left(\left\|v_{n}-v\right\|_{\mathcal{H}^{0, \delta}}+\left\|v_{n}-v\right\|_{\mathcal{H}_{p}^{0,0}}^{p-1}\right) .
\end{aligned}
$$


Therefore, $G v_{n} \rightarrow G v$ in $\mathfrak{X}^{*}$, so $G$ is compact.

Using the fact that $g$ is increasing, one knows

$$
\langle G u, u\rangle \geq 0, \forall u \in \mathfrak{X} .
$$

Furthermore, $F$ is coercive in $\mathfrak{B}$. Hence, $H=F+G$ is also coercive in $\mathfrak{B}$.

Let us define an operator $\Phi: \mathfrak{X} \times \mathfrak{X} \rightarrow \mathfrak{X}^{*}$ which is given by

$$
\langle\Phi(u, w), v\rangle:=\langle F u, v\rangle+\langle G w, v\rangle \text { for all } u, v, w \in \mathfrak{X} .
$$

Then it is straightforward that $\Phi(u, u)=H u$.

We verify now all conditions in Leray-Lions Theorem 2.22

(v) this property holds because $\Phi\left(u+t_{n} h, w\right) \rightarrow \Phi(u, w)$ actually means that $F\left(u+t_{n} h\right) \rightarrow$ $F(u)$. The latter convergence is true due to continuity of $F$.

(vi) for $u, w \in \mathfrak{X}$, we have

$$
\langle\Phi(u, u)-\Phi(w, u), u-w\rangle=\langle F u-F w, u-w\rangle \geq 0,
$$

due to monotonicity of $F$.

(vii) Given a sequence $\left\{v_{n}\right\}$ which converges weakly to $v$ in $\mathfrak{X}$, where $v_{n}, v \in C_{c}^{\infty}(X \backslash Y)$, and assume that

$$
\lim _{n \rightarrow \infty}\left\langle\Phi\left(v_{n}, v_{n}\right)-\Phi(v, v), v_{n}-v\right\rangle=0
$$

Equivalently,

$$
\left\langle F v_{n}-F v, v_{n}-v\right\rangle \rightarrow 0, \text { as } n \rightarrow \infty
$$

The latter is

$$
\begin{aligned}
\left\langle F v_{n}-F v, v_{n}-v\right\rangle= & \int_{X} \nabla\left(v_{n}-v\right)\left[\left|\nabla\left(e+v_{n}\right)\right|^{p-2} \nabla\left(e+v_{n}\right)-\right. \\
& \left.-|\nabla(e+v)|^{p-2} \nabla(e+v)\right] d g \geq \int_{X}\left|\nabla v_{n}-\nabla v\right|^{p} d g \text { use (2.17). }
\end{aligned}
$$

Therefore, $\left\|v_{n}-v\right\|_{2} \rightarrow 0$ as $n \rightarrow \infty$.

Now we use again (2.10) and (2.14) for vectors that for $p \geq 2$ :

$$
|\nabla(e+v)|^{p-2} \geq|\nabla e|^{p-2}-|\nabla v|^{p-2},
$$


which yields

$$
\begin{aligned}
\left\langle F v_{n}-F v, v_{n}-v\right\rangle & =\int_{X}\left[2|\nabla e|^{p-2}-|\nabla v|^{p-2}-\left|\nabla v_{n}\right|^{p-2}\right]\left|\nabla\left(v_{n}-v\right)\right|^{2} d g \\
\geq 2 & \int_{X}|\nabla e|^{p-2}\left|\nabla\left(v-v_{n}\right)\right|^{2} d g-\left\|v-v_{n}\right\|_{2}^{2 / p} \times\left(\|v\|_{2}^{p-2 / p}+\left\|v_{n}\right\|_{2}^{p-2 / p}\right) .
\end{aligned}
$$

Because $v_{n} \rightarrow v$ in $\mathfrak{X}$, the sequence $\left\{\left\|v_{n}\right\|_{2}\right\}$ is bounded and

$$
\lim _{n \rightarrow \infty}\left\langle H v, v_{n}-v\right\rangle=0
$$

Passing to the limit in the equality (3.20), one concludes $\left\|v-v_{n}\right\|_{1} \rightarrow 0$ as $n \rightarrow \infty$. Restricting the operator $H$ to $\mathfrak{B}$, using the equivalence of norms 3.15 , the fact that the seminorms $\left\|v_{n}-v\right\|_{1}$ and $\left\|v_{n}-v\right\|_{2}$ tend to 0 as $n \rightarrow \infty$ implies that $v_{n} \rightarrow v$ in $\mathfrak{B}$. Next, we use continuity of $G$ to obtain the conclusion.

(viii) Let $w \in \mathfrak{X}, u_{n} \rightarrow u$ in $\mathfrak{X}$, and $\Phi\left(w, u_{n}\right) \rightarrow z$.

Because $G$ is compact, the weak convergence $u_{n} \rightarrow u$ implies that $G u_{n} \rightarrow G u$ in $\mathfrak{X}^{*}$ and $\left\{u_{n}\right\}$ is bounded. Therefore, taking an arbitrary function $v \in \mathfrak{X}$, we have

$$
\left\langle\Phi\left(w, u_{n}\right), v\right\rangle=\langle F w, v\rangle+\left\langle G u_{n}, v\right\rangle \rightarrow\langle F w+G u, v\rangle .
$$

By assumption, $z=F w+G u$. Moreover,

$$
\left\langle\Phi\left(w, u_{n}\right), u_{n}\right\rangle=\left\langle F w+G u_{n}, u_{n}\right\rangle \rightarrow\langle F w+G u, u\rangle=\langle z, u\rangle \text { as } n \rightarrow \infty .
$$

Applying Leray-Lions theorem to the operator $\widetilde{H}=\left.H\right|_{\mathfrak{B}}$ and $\widetilde{\Phi}=\left.\Phi\right|_{\mathfrak{B} \times \mathfrak{B}}$, the equation $\widetilde{H}=f$ has at least one solution for all $f \in \mathfrak{B}^{*}$.

\subsection{Second-order quasilinear elliptic equations}

In this section, we look for singular solutions of general second-order quasilinear elliptic equations of the form 3.1 :

$$
-\operatorname{div} \mathcal{F}(x, \nabla u)-\operatorname{div} g_{1}(x, u)+g_{2}(x, u, \nabla u)=f(x), x \in X \backslash Y .
$$

We aim at finding a singular solution to 3.1) under the following structural growth of the nonlinearities: 
(A) $\mathcal{F}(x, q): X \times{ }^{c} T X \rightarrow{ }^{c} T X$ is differentiable in the variables $\left(q_{1}, \ldots, q_{N}\right)$, it is measurable in the first component. Moreover, for all $\left(x, q_{i}\right) \in X \times{ }^{c} T X, i=1,2$, it holds

$$
\left\langle\mathcal{F}\left(x, q_{1}\right)-\mathcal{F}\left(x, q_{2}\right), q_{1}-q_{2}\right\rangle \geq C_{1}\left(\left|q_{1}\right|^{p-2}+\left|q_{2}\right|^{p-2}\right)\left|q_{1}-q_{2}\right|^{2}
$$

and

$$
\left|\mathcal{F}\left(x, q_{1}\right)-\mathcal{F}\left(x, q_{2}\right)\right| \leq C_{2}\left(\left|q_{1}\right|^{p-2}+\left|q_{2}\right|^{p-2}\right)\left|q_{1}-q_{2}\right|
$$

(B) $g_{1}(x, u): X \times \mathbb{R} \rightarrow{ }^{c} T X$ is measurable with respect to the measure $d g$ and

$$
\left|g_{1}\left(x, u_{1}\right)-g_{1}\left(x, u_{2}\right)\right| \leq C\left(\left|u_{1}\right|^{m_{1}-1-\varepsilon}+\left|u_{2}\right|^{m_{1}-1-\varepsilon}\right)\left|u_{1}-u_{2}\right|^{\sigma}
$$

(C) $g_{2}(x, u, q): X \times \mathbb{R} \times{ }^{c} T X \rightarrow{ }^{c} T X$ fulfills

$$
\begin{aligned}
\left|g_{2}\left(x, u_{1}, q_{1}\right)-g_{2}\left(x, u_{2}, q_{2}\right)\right| \leq C\left(\left|u_{1}\right|^{m_{2}-1-\varepsilon}+\left|u_{2}\right|^{m_{2}-1-\varepsilon}\right)\left|u_{1}-u_{2}\right|^{\sigma} & \\
& +\left(\left|q_{1}\right|^{m_{3}-1-\varepsilon}+\left|q_{2}\right|^{m_{3}-1-\varepsilon}\right)\left|q_{1}-q_{2}\right|^{\sigma}
\end{aligned}
$$

where

$$
\begin{aligned}
& m_{1}=\frac{N(p-1)}{N+1-p}+\frac{1-\sigma}{2} \\
& m_{2}=\frac{(N+1)(p-1)}{N+1-p}+\frac{1-\sigma}{2} \\
& m_{3}=\frac{(N+1)(p-1)}{N}+\frac{1-\sigma}{2} \frac{N+p-1}{N}
\end{aligned}
$$

$0<\varepsilon<\min \left\{m_{1}, m_{2}, m_{3}\right\}-1$, and $0 \leq \sigma \leq 1$ are arbitrary numbers.

Remark 3.12. There are restrictions on the growth of all nonlinearities in comparison with the general case of finite energy solutions introduced in [10, 38] due to the fact that the regular part of the solution is controlled by two norms of $\mathcal{H}_{p}^{1,1}(X)$ and $\mathcal{H}^{1, \gamma}(X)$.

Assume that we have known the existence of a formal solution $e$, we will find the regular part $v$ again in the space $\mathfrak{X}$.

Let us recall the embeddings

$$
\mathcal{H}_{p}^{1,1}(X) \hookrightarrow \mathcal{H}_{p^{*}}^{0,0}(X), \mathcal{H}^{1, \gamma}(X) \hookrightarrow \mathcal{H}_{2^{*}}^{0, \gamma-1}(X)
$$

and the compact embeddings

$$
\mathcal{H}_{p}^{1,1}(X) \hookrightarrow \hookrightarrow \mathcal{H}_{q_{1}}^{0,0}(X), \mathcal{H}^{1, \gamma}(X) \hookrightarrow \hookrightarrow \mathcal{H}_{q_{2}}^{0, \gamma-1}(X)
$$


where

$$
p^{*}=\frac{(N+1) p}{N+1-p}, \quad 2^{*}=\frac{2(N+1)}{N-1}
$$

are the critical Sobolev exponents, and $1 \leq q_{1}<p^{*}, 1 \leq q_{2}<2^{*}$.

We define the following operators $\mathbf{F}, \mathbf{G}: \mathfrak{X} \rightarrow \mathfrak{X}^{*}$ by the formulae

$$
\begin{aligned}
\mathbf{F} v & =-\operatorname{div} \mathcal{F}(x, \nabla e+\nabla v)+\operatorname{div} \mathcal{F}(x, \nabla e), \\
\mathbf{G}_{1} v & =-\operatorname{div} g_{1}(x, e+v)+\operatorname{div} g_{1}(x, e), \\
\mathbf{G}_{2} v & =g_{2}(x, e+v, \nabla e+\nabla v)-g_{2}(x, e, \nabla e), \\
\mathbf{G} v & =\mathbf{G}_{1} v+\mathbf{G}_{2} v .
\end{aligned}
$$

For an element $w \in C_{c}^{\infty}(X \backslash Y)$, we can rewrite the actions of $\mathbf{F}$ and $\mathbf{G}$ as follows

$$
\begin{aligned}
\langle\mathbf{F} v, w\rangle & =\int_{X}[\mathcal{F}(x, \nabla e+\nabla v)-\mathcal{F}(x, \nabla e)] \nabla w d g, \\
\left\langle\mathbf{G}_{1} v, w\right\rangle & =\int_{X}\left[g_{1}(x, e+v)-g_{1}(x, e)\right] \nabla w d g, \\
\left\langle\mathbf{G}_{2} v, w\right\rangle & =\int_{X}\left[g_{2}(x, e+v, \nabla e+\nabla v)-g_{2}(x, e, \nabla e)\right] w d g .
\end{aligned}
$$

Now we will show that $\mathbf{F}, \mathbf{G}_{1}, \mathbf{G}_{2}$ are well-defined on $\mathfrak{X}$, bounded and continuous. First, let $w \in C_{c}^{\infty}(X \backslash Y)$ be a test function, we have

$$
\begin{aligned}
|\langle\mathbf{F} v, w\rangle| & =\left|\int_{X}[\mathcal{F}(x, \nabla e+\nabla v)-\mathcal{F}(x, \nabla e)] \nabla w d g\right| \\
& \leq C \int_{X}\left(|\nabla(e+v)|^{p-2}+|\nabla e|^{p-2}\right)|\nabla v \| \nabla w| d g, \text { use (3.23) } \\
& \left.\leq C \int_{X}\left(|\nabla e|^{p-2}+|\nabla v|^{p-2}\right)|\nabla v \| \nabla w| d g, \text { use } 2.14\right) \\
& \leq C\left(\|\nabla v\|_{\mathcal{H}^{0, \gamma-1}}\|\nabla w\|_{\mathcal{H}^{0, \gamma-1}}+\|\nabla v\|_{\mathcal{H}_{p}^{0,0}}^{p-1}\|\nabla w\|_{\mathcal{H}_{p}^{0,0}}\right) \\
& \leq C\left(\|v\|_{\mathfrak{X}}\right)\|w\|_{\mathfrak{X}}
\end{aligned}
$$

This estimate implies that $\mathbf{F}: \mathfrak{X} \rightarrow \mathfrak{X}^{*}$ is well-defined and it is also a bounded operator.

By a similar proof, we can show that $\mathbf{F}$ is continuous. Given a sequence $\left\{v_{n}\right\}$ which converges to $v$ in $\mathfrak{X}$, we have for all $w \in C_{c}^{\infty}(X \backslash Y)$

$$
\begin{aligned}
\left|\left\langle\mathbf{F} v-\mathbf{F} v_{n}, w\right\rangle\right| v & =\left|\int_{X}\left[\mathcal{F}(x, \nabla e+\nabla v)-\mathcal{F}\left(x, \nabla e+\nabla v_{n}\right)\right] \nabla w d g\right| \\
& \leq C\left(\left\|\nabla\left(v-v_{n}\right)\right\|_{\mathcal{H}^{0, \gamma-1}}\|\nabla w\|_{\mathcal{H}^{0, \gamma-1}}+\left\|\nabla\left(v-v_{n}\right)\right\|_{\mathcal{H}_{p}^{0,0}}^{p-1}\|\nabla w\|_{\mathcal{H}_{p}^{0,0}}\right)
\end{aligned}
$$

Using density argument and convergence of $v_{n}$ to $v$, we obtain

$$
\lim _{n \rightarrow \infty}\left\langle\mathbf{F} v-\mathbf{F} v_{n}, w\right\rangle=0 .
$$


Furthermore, the condition (3.22) guarantees that the operator $\mathbf{F}$ has property $(S)_{+}$in the subset $\mathfrak{B}$ of $\mathfrak{X}$. Indeed, taking an arbitrary sequence $\left\{v_{n}\right\}$ which converges weakly to $v$ in $\mathfrak{X}$, and that

$$
\limsup _{n \rightarrow \infty}\left\langle\mathbf{F} v_{n}, v_{n}-v\right\rangle \leq 0 .
$$

By approximation we can assume that $v_{n}$ and $v$ belong to $C_{c}^{\infty}(X \backslash Y)$. The weak convergence $v_{n} \rightarrow v$ also gives us

$$
\limsup _{n \rightarrow \infty}\left\langle\mathbf{F} v_{n}-\mathbf{F} v, v_{n}-v\right\rangle \leq 0 .
$$

The pairing is estimated as follows

$$
\begin{aligned}
\left\langle\mathbf{F} v_{n}-\mathbf{F} v, v_{n}-v\right\rangle & =\int_{X}\left[\mathcal{F}\left(x, \nabla e+\nabla v_{n}\right)-\mathcal{F}(x, \nabla e+\nabla v)\right] \nabla\left(v_{n}-v\right) d g \\
& \geq \int_{X}\left|\nabla v_{n}-\nabla v\right|^{2}\left(\left|\nabla e+\nabla v_{n}\right|^{p-2}+|\nabla e+\nabla v|^{p-2}\right) d g
\end{aligned}
$$

Now we use again the facts that

$$
\left|\nabla e+\nabla v_{n}\right|^{p-2}+|\nabla e+\nabla v|^{p-2} \geq\left|\nabla v_{n}-\nabla v\right|^{p-2}
$$

which yields that $\nabla v_{n} \rightarrow \nabla v$ in $\left(\mathcal{H}_{p}^{0,0}(X)\right)^{N+1}$ as $n \rightarrow \infty$.

Again we can estimate

$$
\left|\nabla e+\nabla v_{n}\right|^{p-2}+|\nabla e+\nabla v|^{p-2} \geq 2|\nabla e|^{p-2}-\left|\nabla v_{n}\right|^{p-2}-|\nabla v|^{p-2} .
$$

Hence,

$$
\begin{aligned}
\left\langle\mathbf{F} v_{n}-\mathbf{F} v, v_{n}-v\right\rangle & \geq \int_{X}\left|\nabla v_{n}-\nabla v\right|^{2}\left[2|\nabla e|^{p-2}-\left|\nabla v_{n}\right|^{p-2}-|\nabla v|^{p-2}\right] d g \\
& \geq\left\|v_{n}-v\right\|_{1}-\left\|v_{n}-v\right\|_{2}^{2}\left(\left\|v_{n}\right\|_{2}^{p-2}+\|v\|_{2}^{p-2}\right) .
\end{aligned}
$$

The last term tends to 0 as $n \rightarrow \infty$ because $\left\|v_{n}\right\|_{2}^{p-2},\|v\|_{2}^{p-2}$ are bounded, $\left\|v_{n}-v\right\|_{2}^{2} \rightarrow 0$. Passing to the limit, we obtain the limit $\nabla v_{n} \rightarrow \nabla v$ in $\mathcal{H}^{0, \gamma-1}(X) \cap \mathcal{H}_{p}^{0,0}(X)$ componentwise as $n \rightarrow \infty$. Therefore, restricting to functions $v \in \mathfrak{B}$, we obtain the convergence $v_{n} \rightarrow v$ in the topology of $\mathfrak{X}$. Hence, we have shown $\widetilde{\mathbf{F}}=\left.\mathbf{F}\right|_{\mathfrak{B}}$ has property $(S)_{+}$.

Next, we verify that the operators $\mathbf{G}_{1}, \mathbf{G}_{2}$ are continuous and compact. We take arbitrary elements $v \in \mathfrak{X}$ and a test function $w \in C_{c}^{\infty}(X \backslash Y)$, it holds

$$
\begin{aligned}
\left|\left\langle\mathbf{G}_{1} v, w\right\rangle\right| & =\left|\int_{X}\left[g_{1}(x, e+v)-g_{1}(x, e)\right] \nabla w d g\right| \\
& \leq C \int_{X}\left(|e+v|^{m_{1}-1-\varepsilon}+|e|^{m_{1}-1-\varepsilon}\right)|v|^{\sigma}|\nabla w| d g \\
& \leq C \int_{X}\left(|e|^{m_{1}-1-\varepsilon}+|v|^{m_{1}-1-\varepsilon}\right)|v|^{\sigma}|\nabla w| d g
\end{aligned}
$$


Using Hölder inequality, we have

$$
\begin{aligned}
& \left.\int_{X}|e|^{m_{1}-1-\varepsilon}|v|\right|^{\sigma}|\nabla w| d g \leq\|v\|_{\mathcal{H}_{2 \sigma}^{0, \delta_{1} / \sigma}}^{\sigma}\left|\|\nabla w \mid\|_{\mathcal{H}^{0, \gamma-1}} \leq\|v\|_{\mathcal{H}^{1, \gamma}}^{\sigma}\|\nabla w\|_{\mathcal{H}^{0, \gamma-1}}\right. \\
& \int_{X}|v|^{m_{1}-1+\sigma-\varepsilon}|\nabla w| d g \leq\|v\|_{\mathcal{H}_{p^{\prime}\left(m_{1}-1+\sigma-\varepsilon\right)}^{m_{1}-1+\sigma-\varepsilon}}^{m_{1}}\|\nabla w \mid\|_{\mathcal{H}_{p}^{0,0}} \leq\|v\|_{\mathcal{H}_{p^{*}}^{0,0}}^{m_{1}-1+\sigma-\varepsilon}\|\nabla w\|_{\mathcal{H}_{p}^{0,0}}
\end{aligned}
$$

where $\delta_{1}=\frac{N+1-p}{p-1}\left(m_{1}-1-\varepsilon\right)-(\gamma-1)$. We have used the embeddings

$$
\mathcal{H}^{1, \gamma}(X) \hookrightarrow \hookrightarrow \mathcal{H}_{2 \sigma}^{0, \delta_{1} / \sigma}(X), \quad \mathcal{H}_{p^{*}}^{0,0}(X) \hookrightarrow \hookrightarrow \mathcal{H}_{p^{\prime}\left(m_{1}-1+\sigma-\varepsilon\right)}^{0,0}(X)
$$

as $\varepsilon>0$. Moreover, these embeddings are compact. It yields that for any sequence $\left\{v_{n}\right\}$ which converges weakly to $v$ in $\mathfrak{X}, v_{n}$ converges strongly to $v$ in $\mathcal{H}_{2 \sigma}^{0, \delta_{1} / \sigma}(X)$ and $\mathcal{H}_{p^{\prime}\left(m_{1}-1+\sigma-\varepsilon\right)}^{0,0}(X)$. By repeating the estimate 3.27$)$, one has

$$
\begin{aligned}
0 \leq\left|\left\langle\mathbf{G}_{1} v_{n}-\mathbf{G}_{1} v, w\right\rangle\right| & =\left|\int_{X}\left[g_{1}\left(x, e+v_{n}\right)-g_{1}(x, e+v)\right] \nabla w d g\right| \\
& \leq\left\|v_{n}-v\right\|_{\mathcal{H}_{2 \sigma}^{0, \delta_{1} / \sigma}}^{\sigma}\|\mid \nabla w\|_{\mathcal{H}^{0, \gamma-1}}+\left\|v_{n}-v\right\|_{\mathcal{H}_{p^{\prime}\left(m_{1}-1+\sigma-\varepsilon\right)}^{0,0}}^{m_{1}-1+\sigma-\varepsilon}\|\nabla w\|_{\mathcal{H}_{p}^{0,0}}
\end{aligned}
$$

Hence, both sides of this inequality converges to 0 as $n \rightarrow \infty$, i.e. $\mathbf{G} v_{n} \rightarrow \mathbf{G} v$ in $\mathfrak{X}^{*}$. So $\mathbf{G}_{1}$ is also a compact operator.

It is also true for the operator $\mathbf{G}_{2}$ that

$$
\begin{aligned}
\left|\left\langle\mathbf{G}_{2} v, w\right\rangle\right| & =\left|\int_{X}\left[g_{2}(x, e+v, \nabla e+\nabla v)-g_{2}(x, e, \nabla e)\right] w d g\right| \\
& \leq C \int_{X}\left(|e+v|^{m_{2}-1-\varepsilon}+|e|^{m_{2}-1-\varepsilon}\right)|v|^{\sigma}|w| d g+ \\
& +\left(|\nabla(e+v)|^{m_{3}-1-\varepsilon}+|\nabla e|^{m_{3}-1-\varepsilon}\right)|\nabla v|^{\sigma}|w| d g, \text { use }[\underline{3.25}) \\
& \leq C \int_{X}\left(|e|^{m_{2}-1-\varepsilon}+|v|^{m_{2}-1-\varepsilon}\right)|v|^{\sigma}|w| d g+\left(|\nabla e|^{m_{3}-1-\varepsilon}+|\nabla v|^{m_{3}-1-\varepsilon}\right)|\nabla v|^{\sigma}|w| d g .
\end{aligned}
$$

Using Hölder inequality, we have

$$
\begin{aligned}
& \int_{X}|e|^{m_{2}-1-\varepsilon}|v|^{\sigma}|w| d g \leq\|v\|_{\mathcal{H}_{\left(2^{*}\right)^{\prime} \sigma}^{0, \delta_{2} / \sigma}}^{\sigma}\|w\|_{\mathcal{H}_{2^{*}}^{0, \gamma-1}} \leq\|v\|_{\mathcal{H}^{1, \gamma}}^{\sigma}\|w\|_{\mathcal{H}^{1, \gamma}} \\
& \int_{X}|v|^{m_{2}-1+\sigma-\varepsilon}|w| d g \leq\|v\|_{\mathcal{H}_{\left(p^{*}\right)^{\prime}\left(m_{2}-1+\sigma-\varepsilon\right)}^{0,0}}^{m_{2}-1+\sigma-\varepsilon} \quad\|w\|_{\mathcal{H}_{p^{*}}^{0,0}} \leq\|v\|_{\mathcal{H}_{p}^{1,1}}^{m_{2}-1+\sigma-\varepsilon}\|w\|_{\mathcal{H}_{p}^{1,1}} \\
& \int_{X}|\nabla v|^{m_{3}-1+\sigma-\varepsilon}|w| d g \leq\|\| \nabla v\left\|_{\mathcal{H}_{\left(p^{*}\right)^{\prime}\left(m_{3}-1+\sigma-\varepsilon\right)}^{0,1+\sigma}}^{m_{3}-1+\sigma-\varepsilon} \quad\right\| w\left\|_{\mathcal{H}_{p^{*}}^{0,0}} \leq\right\| \nabla v\left\|_{\mathcal{H}_{p}^{0,0}}^{m_{3}-1+\sigma-\varepsilon}\right\| w \|_{\mathcal{H}_{p}^{1,1}} \\
& \int_{X}|\nabla e|^{m_{3}-1-\varepsilon}|\nabla v|^{\sigma}|w| d g \leq\|\| \nabla v\left\|_{\mathcal{H}_{\left(2^{*}\right)^{\prime} \sigma}^{0, \delta_{3} / \sigma}}^{\sigma}\right\| w\left\|_{\mathcal{H}_{2^{*}}^{0, \gamma-1}} \leq\right\| \mid \nabla v\|\|_{\mathcal{H}^{0, \gamma-1}}^{\sigma}\|w\|_{\mathcal{H}^{1, \gamma}}
\end{aligned}
$$

where

$$
\delta_{2}=\left(m_{2}-1-\varepsilon\right) \frac{N+1-p}{(p-1)}-(\gamma-1), \quad \delta_{3}=\frac{\left(m_{3}-1-\varepsilon\right) N}{p-1}+1-\gamma
$$


which guarantee the embeddings

$$
\mathcal{H}^{1, \gamma}(X) \hookrightarrow \mathcal{H}_{\left(2^{*}\right)^{\prime} \sigma}^{0, \delta_{2} \sigma}(X), \quad \mathcal{H}^{0, \gamma-1}(X) \hookrightarrow \mathcal{H}_{\left(2^{*}\right)^{\prime} \sigma}^{0, \delta_{3} \sigma}(X)
$$

The embeddings used for $v$ are compact, due to the choice of $\varepsilon>0$, we conclude that $\mathbf{G}_{2}$ is a compact operator.

Because the $(S)_{+}$property is stable under compact perturbations, the operator $\mathbf{F}+\mathbf{G}$ has also this property on the subset $\mathfrak{B}$ of $\mathfrak{X}$. So we have proved the following result.

Theorem 3.13. Under the conditions (3.22), (3.23), (3.24), 3.25), the operator $\mathbf{F}+$ $\mathbf{G}: \mathfrak{X} \rightarrow \mathfrak{X}^{*}$ possesses the property $(S)_{+}$.

In the next part, we will examine several cases when the mapping degree is nonzero, hence existence holds. Our consideration here includes the two existence theorems mentioned at the beginning of this section in the setting of a manifold with conic points.

\subsubsection{Serrin's case}

We refer to Serrin's case the equations with lower order terms whose growth is smaller than the one of the principal part, namely $m_{1}, m_{2}, m_{3}<p-1$. Moreover, we require that $0 \leq \sigma<1$.

Using again the structure assumptions (3.22), 3.25), we estimate first for an element $v \in C_{c}^{\infty}(X \backslash Y)$

$$
\begin{aligned}
\langle(\mathbf{F}+\mathbf{G}) v, v\rangle \geq C_{0}\left(\|\nabla v\|_{\mathcal{H}_{p}^{0,0}}^{p}+\|\nabla v\|_{\mathcal{H}^{0, \gamma-1}}^{2}\right)-C_{1}\|v\|_{\mathcal{H}^{1, \gamma}}^{\sigma+1}- & \\
& -C_{2}\left(\|v\|_{\mathcal{H}_{p}^{1,1}}^{m_{1}+\sigma-\varepsilon}+\|v\|_{\mathcal{H}_{p}^{1,1}}^{m_{2}+\sigma-\varepsilon}+\|v\|_{\mathcal{H}_{p}^{1,1}}^{m_{3}+\sigma-\varepsilon}\right)
\end{aligned}
$$

This inequality holds also for $u \in \mathfrak{X}$ due to density of $C_{c}^{\infty}(X \backslash Y)$ in $\mathfrak{X}$. Now we restrict this estimate to $v \in \mathfrak{B}$, it is true that

$$
\begin{aligned}
\langle(\mathbf{F}+\mathbf{G}) v, v\rangle \geq C_{0}\left(\|v\|_{\mathcal{H}_{p}^{1,1}}^{p}+\|v\|_{\mathcal{H}^{1, \gamma}}^{2}\right)- & C_{1}\|v\|_{\mathcal{H}^{1, \gamma}}^{\sigma+1}- \\
& -C_{2}\left(\|v\|_{\mathcal{H}_{p}^{1,1}}^{m_{1}+\sigma-\varepsilon}+\|v\|_{\mathcal{H}_{p}^{1,1}}^{m_{2}+\sigma-\varepsilon}+\|v\|_{\mathcal{H}_{p}^{1,1}}^{m_{3}+\sigma-\varepsilon}\right)
\end{aligned}
$$

We have put assumptions that $\sigma<1$ and $m_{1}, m_{2}, m_{3}<p-1$, therefore, when $v \in V$ with $\|v\|_{\mathfrak{X}} \rightarrow \infty$, either the norm $\|v\|_{\mathcal{H}_{p}^{1,1}}$ or the norm $\|v\|_{\mathcal{H}^{1, \gamma}}$ tends to infinity, the first two terms always dominate the other and dominate also $\|v\|_{\mathfrak{X}}$. Therefore, we conclude that

$$
\lim _{\substack{v \in \mathfrak{B} \\\|v\|_{\mathfrak{X} \rightarrow \infty}}} \frac{\langle(\mathbf{F}+\mathbf{G}) v, v\rangle}{\|v\|_{\mathfrak{X}}}=\infty .
$$


There exists a number $R>0$ such that for all $v \in \mathfrak{B}$ and $\|v\|_{\mathfrak{X}} \geq R$, it holds

$$
\frac{\langle(\mathbf{F}+\mathbf{G}) v, v\rangle}{\|v\|_{\mathfrak{X}}} \geq\|f\|_{\mathfrak{X}^{*}}
$$

Consequently, we can assign to the mapping $\mathbf{F}+\mathbf{G}$ a degree on the ball $B(0, R)$ in $\mathfrak{B}$ with respect to $f \in \mathfrak{B}^{*}$. Furthermore, by lemma 2.31 this degree $\operatorname{Deg}(\mathbf{F}+\mathbf{G}, B(0, R), f)=1$. The existence of the equation (3.1) then follows.

We have proved the following result:

Theorem 3.14. Let $p \geq 2$ and assume that the nonlinearities $\mathcal{F}, g_{1}$, and $g_{2}$ satisfy the conditions (3.22), (3.23), (3.24), (3.25), where $m_{1}, m_{2}, m_{3}<p-1$ and $\sigma<1$. Then given an $f \in \mathfrak{B}^{*}$, the equations (3.1) has a singular solution of the form $e+v$, where $e \sim c t^{-\alpha}$ as $t \rightarrow 0$ and $v \in \mathfrak{B}$.

\subsubsection{Equations with an absorption term $g(u)$}

We refer this case to the situation that $g_{1}(u)=0$ and $g_{2}(x, u, \nabla u)=g_{2}(u)$. One example is the case $g_{2}(u)=|u|^{q-1} u$, where $p-1<q<\frac{(N+1)(p-1)}{N+1-p}$. Our result extends the one in [12].

Theorem 3.15. Assume that the nonlinearity $g$ is an increasing function with respect to the second variable. Given an $f \in \mathfrak{B}^{*}$, there exists a solution to 3.17) which is of the form $e+v$, where $e \sim C t^{-\alpha}$ and $v \in \mathfrak{B} \subset \mathfrak{X}$.

Proof. We will verify that the operator $\mathbf{F}+\mathbf{G}$ is coercive. Indeed, taking an arbitrary element $u \in C_{c}^{\infty}(X \backslash Y)$, we have

$$
\langle\mathbf{G} u, u\rangle=\int_{X}\left(g_{2}(e+u)-g_{2}(e)\right) u d g \geq 0,
$$

because $g_{2}$ is an increasing function. Therefore, by density argument we also obtain that $\langle\mathbf{G} u, u\rangle \geq 0$ for all $u \in \mathfrak{X}$. Combining with the fact that $\mathbf{F}$ is coercive, so is $\mathbf{F}+\mathbf{G}$. Then we repeat the argument in the theorem above and conclude that this mapping has a non-zero degree on a large enough ball $B(0, R) \subset \mathfrak{B}$. So existence is obvious.

\subsubsection{General assumption about coercivity}

Theorem 3.16. Let $p \geq 2$ and assume that for $\left(x, u_{1}, q_{1}\right),\left(x, u_{2}, q_{2}\right) \in X \times \mathbb{R} \times^{c} T X$, it holds

$$
\begin{aligned}
\left\langle\mathcal{F}\left(x, q_{1}\right)-\right. & \left.\mathcal{F}\left(x, q_{2}\right), q_{1}-q_{2}\right\rangle+\left\langle g_{1}\left(x, u_{1}\right)-g_{1}\left(x, u_{2}\right), q_{1}-q_{2}\right\rangle \\
& +\left\langle g_{2}\left(x, u_{1}, q_{1}\right)-g_{2}\left(x, u_{2}, q_{2}\right), u_{1}-u_{2}\right\rangle \geq\left(\left|q_{1}\right|^{p-2}+\left|q_{2}\right|^{p-2}\right)\left|q_{1}-q_{2}\right|^{2}
\end{aligned}
$$


Then for all $f \in \mathfrak{B}^{*}$, the equation (3.1) will have at least a solution $e+v$, where $v \in \mathfrak{B}$.

Proof. It remains to show that the operator is coercive, which follows from the growth condition (3.30). Indeed, we estimate first for all $u \in C_{c}^{\infty}(X \backslash Y)$

$$
\begin{aligned}
\left|\langle\mathbf{F} v, v\rangle+\left\langle\mathbf{G}_{1} v, v\right\rangle+\left\langle\mathbf{G}_{2} v, v\right\rangle\right| C & \geq \int_{X}\left(|\nabla e+\nabla v|^{p-2}+|\nabla e|^{p-2}\right)|\nabla v|^{2} d g \\
& \geq C \int_{X}\left(|\nabla e|^{p-2}|\nabla v|^{2}+|\nabla v|^{p}\right) d g
\end{aligned}
$$

Hence, the operator $\mathbf{F}+\mathbf{G}_{1}+\mathbf{G}_{2}$ is coercive operator in $\mathfrak{X}$, and it induces a coercive operator in $\mathfrak{B}$. Repeating the argument in the two theorems above, this operator has nonzero degree on a large enough ball $B(0, R) \subset \mathfrak{B}$. Existence then follows. 


\section{Higher-order quasilinear elliptic equations}

In this chapter, we will be concerned about higher-order quasilinear elliptic equations. As we have mentioned, in higher-order case, the methods of comparison principle and truncation no longer work. We will approach existence questions by using nonlinear functional analysis.

Equations in divergent form have some monotone property. One knows from theory of monotone operators the fact that we can enlarge the class of lower order terms in investigation when one requires more general properties of the principal part. For a detailed and careful look into quasilinear elliptic equations in smooth domains, we refer to the monograph of Drábek, Kufner, Nicolosi [9]. So far, there have been no existence results in the singular manifolds setting.

Let us also emphasize our approach to verify existence. We do not follow the usual procedure, namely start with a problem itself and try to work out in which function spaces the solutions exist. Instead we will fix in advance a function space $V$ then set up appropriate problems (i.e. the operator $A$ and the nonlinearities in consideration) to which the theory is applicable. Therefore, we verify some important properties of functions belonging to a certain maximal domain $D\left(A_{\max }^{\gamma, p}\right)$ of a given general cone-degenerate differential operator $A$ and some embedding theorems.

We begin this chapter with the cone calculus, which is mostly taken from the books of Egorov-Schulze [11] and of Schulze [33] for $L^{2}$ theory; and the paper [31] of SeilerSchrohe on $L^{p}$ theory. Using knowledge of parametrices, we can prove that in the case $A^{\prime} A$ is an elliptic operator, $D\left(A_{\min }\right)$ also has finite codimension in $D\left(A_{\max }\right)$. This combines with the fact that $D\left(A_{\min }\right)$ is compacty embedded in $\mathcal{H}_{p}^{0, \gamma}(X)$ also gives us the compact embedding $D\left(A_{\max }\right) \hookrightarrow \hookrightarrow \mathcal{H}_{p}^{0, \gamma}(X)$. We show then the existence of finite energy solutions and singular solutions. The key point is again the assumption about the existence of 
a formal solution or equivalently the solvability of corresponding nonlinear eigenvalue problems, see Equation (4.26).

The need for establishing existence of solutions of higher-order equations is certainly of mathematical interest. Moreover, there are also meaningful models in elasticity which serve as examples of the equations we are interested in. Let us recall two examples in [38, Chapter 1]. The first one is the model of the elasto-plastic bending of a rigidly clamped plate. The deflection of the plate fulfills the equation

$$
\begin{aligned}
\frac{\partial^{2}}{\partial x^{2}}\left[g\left(H^{2}(u)\right)\left(\frac{\partial^{2} u}{\partial x^{2}}+\frac{1}{2} \frac{\partial^{2} u}{\partial y^{2}}\right)\right]+\frac{\partial^{2}}{\partial x \partial y}\left[g\left(H^{2}(u)\right) \frac{\partial^{2} u}{\partial x \partial y}\right] \\
+\frac{\partial^{2}}{\partial y^{2}}\left[g\left(H^{2}(u)\right)\left(\frac{\partial^{2} u}{\partial y^{2}}+\frac{1}{2} \frac{\partial^{2} u}{\partial x^{2}}\right)\right]=f(x, y),(x, y) \in \Omega \subset \mathbb{R}^{2},
\end{aligned}
$$

and the boundary conditions

$$
\left.u\right|_{\partial \Omega}=\left.\frac{\partial u}{\partial n}\right|_{\partial \Omega}=0
$$

where

$$
H^{2}(u)=\left(\frac{\partial^{2} u}{\partial x^{2}}\right)^{2}+\left(\frac{\partial^{2} u}{\partial y^{2}}\right)^{2}+\left(\frac{\partial^{2} u}{\partial x \partial y}\right)^{2}+\frac{\partial^{2} u}{\partial x^{2}} \frac{\partial^{2} u}{\partial y^{2}}
$$

and $g(t)$ is a function characterizing the given material.

The second example is the system of equations of strong bending of thin plates. The deflection $u$ and the stress function $F$ satisfy

$$
\begin{aligned}
& \frac{D}{h} \Delta^{2} u-L(u, F)=\frac{1}{h} q(x, y), \\
& \frac{2}{E} \Delta^{2} F+L(u, u)=0,(x, y) \in \Omega \subset \mathbb{R}^{2}, \\
& \left.u\right|_{\partial \Omega}=\left.\frac{\partial u}{\partial n}\right|_{\partial \Omega}=\left.F\right|_{\partial \Omega}=\left.\frac{\partial F}{\partial n}\right|_{\partial \Omega}=0,
\end{aligned}
$$

where $h, E$, and $D$ are constants, $\Delta$ is the Laplacian and

$$
L(u, F)=\frac{\partial^{2} u}{\partial x^{2}} \frac{\partial^{2} F}{\partial y^{2}}+\frac{\partial^{2} u}{\partial y^{2}} \frac{\partial^{2} F}{\partial x^{2}}-2 \frac{\partial^{2} u}{\partial x \partial y} \frac{\partial^{2} F}{\partial x \partial y} .
$$

\subsection{Cone algebra with asymptotics $C^{v}(X, \mathfrak{g})$}

We have summarized in chapter 1 the definitions of cone-degenerate differential operators and of weighted Sobolev spaces, and some properties of these operators acting between these spaces including continuity and ellipticity. In this chapter, we summarize main results in the cone algebra with asymptotics. It contains another scale of so-called cone Sobolev spaces with asymptotics. An operator in this calculus is associated with two 
symbols whose invertibility is equal to ellipticity of the operator, and the ellipticity is equivalent to Fredholm property of the operator acting between cone Sobolev spaces with asymptotics. All statements are correct in the bundle case, however, for simple notation, we will suspend indicating the bundle in this exposition.

\subsubsection{Mellin transformation}

Let us recall the form of cone-degenerate differential operator (2.1). The appearance of the derivative $-t \partial_{t}$ in the formula motivates us to use the Mellin transform, which maps each time derivative with respect to $-t \partial_{t}$ to $z$. Moreover, it is shown in [11, 7.2.3, Proposition 1] that we can employ the Mellin transform to quantize operators on manifolds with singularities. The following is the Mellin transform for scalar functions belonging to $C_{c}^{\infty}\left(\mathbb{R}_{+}\right)$.

Definition 4.1. Let $u(t) \in C_{c}^{\infty}\left(\mathbb{R}_{+}\right), z \in \mathbb{C}$, then the Mellin transform of $u$ is defined by the following formula

$$
\mathcal{M} u(z)=\int_{0}^{\infty} t^{z-1} u(t) d t
$$

and the image is an entire function $\mathcal{M} u(z) \in \mathcal{A}(\mathbb{C})$.

Here are some important properties of this transform:

Proposition 4.2. For $t \in \mathbb{R}_{+}, z, p \in \mathbb{C}, \beta \in \mathbb{R} \backslash\{0\}$, and $u \in C_{0}^{\infty}\left(\mathbb{R}_{+}\right)$, we have

$$
\begin{aligned}
\mathcal{M}\left(-t \partial_{t} u\right)(z) & =z \mathcal{M} u(z), \\
\mathcal{M}\left(t^{-p} u\right)(z) & =(\mathcal{M} u)(z-p), \\
\mathcal{M}((\log t) u)(z) & =\left(\partial_{z} \mathcal{M} u\right)(z), \\
\mathcal{M}\left(u\left(t^{\beta}\right)\right)(z) & =\beta^{-1}(\mathcal{M} u)\left(\beta^{-1} z\right) .
\end{aligned}
$$

The Mellin transform can be extended to more general spaces of distributions on $\mathbb{R}_{+}$, namely to the weighted space $L^{2, \gamma}\left(\mathbb{R}_{+}\right)=t^{\gamma} L_{2}\left(\mathbb{R}_{+}\right)$for a weight $\gamma \in \mathbb{R}$. We notice the following important link between the Mellin and Fourier transforms.

Let us define $S_{\gamma} u(t)=e^{-(1 / 2-\gamma) t} u\left(e^{-t}\right)$. Then $S_{\gamma}: C_{c}^{\infty}\left(\mathbb{R}_{+}\right) \rightarrow C_{c}^{\infty}(\mathbb{R})$ is an isomorphism. Therefore, $S_{\gamma}$ extends to an isomorphism

$$
S_{\gamma}: t^{\gamma} L^{2}\left(\mathbb{R}_{+}\right) \rightarrow L^{2}(\mathbb{R})
$$


We already know that the Fourier transform is an isomorphism between the following $L^{2}$ spaces

$$
\mathcal{F}: L^{2}(\mathbb{R}, d t) \rightarrow L^{2}(\mathbb{R}, d \rho)
$$

where $\mathcal{F} v(\rho)=\int_{\mathbb{R}} e^{-i t \rho} d t$. Composing these maps, we obtain an isomorphism

$$
\mathcal{F} S_{\gamma}: t^{\gamma} L^{2}\left(\mathbb{R}_{+}\right) \rightarrow L^{2}\left(\mathbb{R}_{\rho}\right)
$$

And simple calculation shows us

$$
\left(\mathcal{F} S_{\gamma} u\right)(\rho)=\mathcal{M} u(1 / 2-\gamma+\mathrm{i} \tau \rho)
$$

Hence, the Mellin transform is an isomorphism

$$
\mathcal{M}: L^{2, \gamma}\left(\mathbb{R}_{+}\right) \rightarrow L^{2}\left(\Gamma_{1 / 2-\gamma}\right) \text {. }
$$

Restricting the Mellin transform to the line $\Gamma_{1 / 2-\gamma}=\{z \in \mathbb{C}: \mathfrak{R} z=1 / 2-\gamma\}$ yields the socalled weighted Mellin transform

$$
\mathcal{M}_{\gamma} u=\left.\mathcal{M} u\right|_{\Gamma_{1 / 2-\gamma}}=\int_{0}^{\infty} t^{1 / 2-\gamma+\mathrm{i} \tau-1} u(t) d t
$$

which is an isomorphism $\mathcal{M}_{\gamma}: L^{2, \gamma}\left(\mathbb{R}_{+}\right) \rightarrow L_{2}\left(\Gamma_{1 / 2-\gamma}\right)$. Its inverse is given by the formula

$$
\mathcal{M}_{\gamma}^{-1} g(t)=\frac{1}{2 \pi \mathrm{i}} \int_{\Gamma_{1 / 2-\gamma}} t^{-z} g(z) d z
$$

\subsubsection{Cone weighted Sobolev spaces with asymptotics}

Besides cone Sobolev spaces, there is another version of those functional spaces which describes additional information about the behavior of those functions when approaching $t=0$. Solutions to linear elliptic equations on manifolds with conic points are proved to possess conormal asymptotic expansions

$$
u(t, y) \sim \sum_{j} \sum_{k=0}^{m_{j}} \psi_{j k}(y) t^{-p_{j}} \ln ^{k} t, \text { as } t \rightarrow 0,
$$

where $p_{j} \in \mathbb{C}$ are singular exponents. The data $\left(p_{j}, m_{j}\right)$ and the space $L_{j}$ containing $\psi_{j k}(y)$ are determined uniquely from the linear operator. Now we recall how asymptotic information is organized and attached to functional spaces.

Given a weight $\gamma \in \mathbb{R}$ and a closed weight interval $\Theta=[\zeta, 0],-\infty \leq \zeta \leq 0$, then the weight data $(\gamma, \Theta)$ associates with the following weight strip

$$
\Gamma_{(\gamma, \Theta)}=\left\{z \in \mathbb{C} \mid \frac{N+1}{p}-\gamma+\zeta<\mathfrak{R} z<\frac{N+1}{p}-\gamma\right\} .
$$


Definition 4.3. (i) An asymptotic type $P$ associated with the finite weight data $(\gamma, \Theta)$, that is $-\infty<\zeta \leq 0$, is a sequence

$$
P=\left\{\left(p_{j}, m_{j}, L_{j}\right) \mid p_{j} \in \Gamma_{(\gamma, \Theta)}, m_{j} \in \mathbb{Z}_{+}, j=0, \ldots, \bar{N}\right\}
$$

for some $\bar{N} \in \mathbb{N}_{0}$, and $L_{j}$ are subspaces of $C^{\infty}(X)$.

In the case of infinite weight $\zeta=-\infty$, one requires further that the real part of the exponents $\mathfrak{R} p_{j} \rightarrow-\infty$ as $j \rightarrow \infty$. We denote by $\operatorname{As}(\gamma, \Theta)$ the set of all discrete asymptotic types associated with the weight data $(\gamma, \Theta)$.

(ii) Given two asymptotic types $P=\left\{\left(p_{j}, m_{j}, L_{j}\right)\right\}$ and $\widetilde{P}=\left\{\left(\widetilde{p}_{k}, \widetilde{m}_{k}, \widetilde{L}_{k}\right)\right\}$. We will write $P \leq \widetilde{P}$ if $\left\{p_{j}\right\} \subset\left\{\widetilde{p}_{k}\right\}$, moreover, if $p_{j}=\widetilde{q}_{k}$ then $m_{j} \leq \widetilde{m}_{k}$ and $L_{j} \subset \widetilde{L}_{k}$.

(iii) Let $P$ be an asymptotic type and $\omega(t)$ be a fixed cut-off function. Let us define

$$
\mathcal{E}_{P}=\left\{\omega(t) \sum_{j=1}^{\bar{N}} \sum_{k=0}^{m_{j}} \psi_{j k}(y) t^{-p_{j}} \log ^{k} t \mid \psi_{j k}(y) \in L_{j}\right\} .
$$

This space has the natural topology of a finite-dimensional space.

(iv) Define the weighted Sobolev spaces with asymptotics as

$$
\mathcal{H}_{p, P}^{s, \gamma}(X)=\mathcal{H}_{p}^{s, \gamma-\zeta}(X)+\mathcal{E}_{P}
$$

The space $\mathcal{H}_{p, P}^{s, \gamma}(X)$ is a Banach space with the topology of non-direct sum of Banach spaces. At weight $\gamma$ that the corresponding weight line contains some poles of the asymptotic type $P$, one uses interpolation to define $\mathcal{H}_{p, P}^{s, \gamma}(X)$.

(v) In case the asymptotic type $P$ associates with an infinite weight interval $(-\infty, 0]$, we define for each $l \in \mathbb{N}$ a finite weight interval $\Theta_{l}=[-l, 0]$ and consider all asymptotic types:

$$
P_{l}=\left\{(p, m, L) \in P \mid p \in \Gamma_{\left(\gamma, \Theta_{l}\right)}\right\}
$$

associated with $\Theta_{l}$. Then we define

$$
\mathcal{H}_{p, P}^{s, \gamma}(X)=\bigcap_{l \in \mathbb{N}} \mathcal{H}_{p, P_{l}}^{s, \gamma}(X)
$$

Its has the Fréchet topology of projective limit. 
Remark 4.4. It is evident that $P_{l} \subset P_{l+1}$ and the embedding $\mathcal{H}_{p, P_{l+1}}^{s, \gamma}(X) \hookrightarrow \mathcal{H}_{p, P_{l}}^{s, \gamma}(X)$ is continuous. Moreover, $\mathcal{H}_{p, P}^{s, \gamma}(X)=\mathcal{H}^{s, \gamma-\zeta}(X)$ when $\max \left\{\mathfrak{R} p_{j}\right\}_{p_{j} \in P}>\gamma-\zeta$, the space $\mathcal{H}_{p, P}^{s, \gamma}(X)$ is well-defined and is again a Banach space.

Remark 4.5. In the case $s, \gamma \in \mathbb{R}, \zeta<0$ (which means that one uses open weight interval), and $P \in \operatorname{As}(\gamma, \Theta)$ where the weight strip is

$$
\Gamma_{(\gamma, \Theta)}=\left\{z \in \mathbb{C} \mid \frac{N+1}{p}-\gamma+\zeta<\mathfrak{R} z<\frac{N+1}{p}-\gamma\right\},
$$

one also considers the spaces

$$
\mathcal{H}_{p, \Theta}^{s, \gamma}(X):=\bigcap_{\varepsilon>0} \mathcal{H}_{p}^{s, \gamma+\zeta-\varepsilon}(X)
$$

These scales of spaces are Fréchet with the projective limit topology. The weighted Sobolev spaces with asymptotics are defined as the sum

$$
\mathcal{H}_{p, P}^{s, \gamma}(X)=\mathcal{H}_{p, \Theta}^{s, \gamma}(X)+\mathcal{E}_{P}
$$

The space $\mathcal{H}_{p, P}^{s, \gamma}(X)$ has the natural Fréchet topology of non-direct sum.

Remark 4.6. In the case $p=2$, weighted Sobolev spaces with asymptotics are Hilbert spaces. Their properties are considered in [27, Section 2.4].

Remark 4.7. The weighted space with asymptotic can be defined invariantly on manifolds when we require further the so-called shadow condition on the asymptotic type, see [19]. An asymptotic type $P \in \operatorname{As}(g)$ for $g=(\gamma, \Theta), \gamma \in \mathbb{R}, \Theta=(-\infty, 0]$ is said to satisfy the shadow condition if

$$
(p, m) \in P \Rightarrow(p-j, m(j)) \in P
$$

for all $j \in \mathbb{N}$ and $m(j) \geq m$. For finite weight interval $\Theta=(\zeta, 0]$ and $\zeta>-\infty$ then (4.6) holds for finite number of $j \in \mathbb{N}$ that $\mathfrak{R} p-j>\frac{N+1}{p}-\gamma+\zeta$.

Example 4.8. A function $u \in C_{0}^{\infty}\left(\overline{\mathbb{R}}_{+}, C^{\infty}(Y)\right), \omega(t) u \in \mathcal{H}^{0, N / 2}(X)$ in the case $\delta=0, p=2$, has Taylor expansion, where $p_{j}=-j, m_{j}=0$ for $j \in \mathbb{N}$.

A function $u \in C_{0}^{\infty}(X)$ which vanishes up to infinite order at the boundary possesses the so-called empty asymptotic, we will denote by $O$.

We list some properties of the spaces with asymptotics $\mathcal{H}_{p, P}^{s, \gamma}(X)$ :

Proposition 4.9. Let $s, s^{\prime} \in \mathbb{R}, \zeta \leq 0, P \in \operatorname{As}(\gamma, \Theta)$. Then

a) $\mathcal{H}_{p, P}^{s, \gamma}(X)=\mathcal{H}_{p}^{s, \gamma}(X)$ when $\zeta=0$ 
b) $\mathcal{H}_{p, O}^{s, \gamma}(X)=\mathcal{H}_{p}^{s, \gamma-\zeta}(X)$.

c) $\mathcal{H}_{p, P}^{s, \gamma}(X)=\mathcal{H}_{p, \widetilde{P}}^{s, \gamma-a}(X)$, where $a>0$ and the asymptotic type $\widetilde{P}$ associates with the weight data $(\gamma-a,[\zeta-a, 0])$.

d) We have $\mathcal{H}_{p, P}^{s, \gamma}(X)=\mathcal{H}_{p, O}^{s, \gamma}(X) \oplus \mathcal{E}_{P}$, provided that $\mathfrak{R} p_{j} \neq \frac{N+1}{p}-\gamma+\zeta$ for all $p_{j} \in P$.

\section{Descriptions of Mellin images of distributions when $p=2$}

It can easily be checked that the function

$$
\varphi(t, y)=\omega t^{-\alpha} \log ^{k} t \psi(y), t>0, \psi(y) \in C^{\infty}(Y)
$$

belongs to $\mathcal{H}_{p}^{s, \gamma}(X)$ if and only if $\mathfrak{R} \alpha<\frac{N+1}{p}-\gamma$.

The following statements are taken from [11, Section 8.1.1].

The Mellin image $\mathcal{M}_{\gamma-\frac{N}{2}} \mathcal{E}_{P} \subset \mathcal{A}\left(\Gamma_{(\gamma, \Theta)} \backslash \pi_{\mathbb{C}} P, C^{\infty}(Y)\right)$. Each element $h(z) \in \mathcal{M}_{\gamma-\frac{N}{2}} \mathcal{E}_{P}$ is meromorphic in the weight strip $\Gamma_{(\gamma, \Theta)}$ with only poles at $p_{j} \in \pi_{\mathbb{C}} P$ of multiplicities $m_{j}+1$. Furthermore, for any $\pi_{\mathbb{C}} P$ - excision function $\chi(z)$, we have that

$$
\left.\chi(z) h(z)\right|_{\Gamma_{\beta}} \in \mathcal{S}\left(\Gamma_{\beta}, C^{\infty}(Y)\right)
$$

holds uniformly in $c \leq \beta \leq c^{\prime}$, for all $c, c^{\prime} \in \mathbb{R}$.

$\mathcal{H}_{2, \Theta}^{s, \gamma}(X)$ is the subspace of all $u \in \mathcal{H}^{s, \gamma}(X)$ whose Mellin image

$$
h(z)=\mathcal{M}_{\gamma-\frac{N}{2}}(\omega u)(z)
$$

is first given on the line $\Gamma_{\frac{(N+1)}{p}-\gamma}$ and has extension to a holomorphic function on the half plane

$$
\left\{z \mid \frac{N+1}{p}-\gamma+\zeta<z\right\} .
$$

Moreover, $h_{\beta}=\left.h\right|_{\Gamma_{\beta}}$ has the following property

$$
\left(\mathcal{F}^{-1} h_{\beta}\right)(t) \in H_{p}^{s}\left(\mathbb{R}^{n+1}\right)
$$

uniformly in $c \leq \beta \leq c^{\prime}$ for every $c<c^{\prime} \in \mathbb{R}$.

\subsubsection{Green operators}

Now we introduce all ingredients for the cone calculus. The residual operators in the cone algebra are Green operators and smoothing Mellin operators. In particular, one can invert an elliptic operator in the cone algebra modulo Green operators, or modulo Green and smoothing Mellin operators. We define these operators by pointing out their mapping properties. 
Definition 4.10 ([34, Definition 3.1]). Let $\mathfrak{g}=(\gamma, \delta, \Theta)$ be a weight data, $\gamma, \delta \in \mathbb{R}, \Theta=$ $(\zeta, 0],-\infty \leq \zeta<0$. Let $P \in \operatorname{As}(\delta, \theta)$ and $Q \in \operatorname{As}(-\gamma, \theta)$ be two asymptotic types. Then the space of Green operators $C_{G}(X, \mathfrak{g})_{P, Q}$ with asymptotic types $P, Q$ is the space of all operators

$$
G \in \bigcap_{s \in \mathbb{R}} \mathcal{L}\left(\mathcal{H}^{s, \gamma}(X), \mathcal{H}^{\infty, \delta}(X)\right)
$$

which for all $s \in \mathbb{R}$ induce continuous operators

$$
\begin{aligned}
& G: \mathcal{H}^{s, \gamma}(X) \rightarrow \mathcal{H}_{P}^{\infty, \delta}(X), \\
& G^{*}: \mathcal{H}^{s,-\delta}(X) \rightarrow \mathcal{H}_{Q}^{\infty,-\gamma}(X),
\end{aligned}
$$

here the adjoint is taken with respect to the inner product of $\mathcal{H}_{2}^{0,0}(d \mu)$.

The space of Green operators is defined as $C_{G}(X, \mathfrak{g})=\bigcup_{P, Q} C_{G}(X, \mathfrak{g})_{P, Q}$.

The subspace $C_{G}(X, \mathfrak{g})_{P, Q}$ is furnished with the projective topologies

$$
\bigcap_{s \in \mathbb{R}} \mathcal{L}\left(\mathcal{H}_{p}^{s, \gamma}(X), \mathcal{H}_{P}^{\infty, \delta}(X)\right), \bigcap_{s \in \mathbb{R}} \mathcal{L}\left(\mathcal{H}_{p^{\prime}}^{s,-\delta}(X), \mathcal{H}_{Q}^{\infty,-\gamma}(X)\right)
$$

Because of property (4.7), every Green operator $G \in C_{G}(X, \mathfrak{g})$ is a compact operator belonging to $\mathcal{L}\left(\mathcal{H}^{s, \gamma}(X), \mathcal{H}^{t, \delta}(X)\right)$ for all $s, t \in \mathbb{R}$.

Green operators can be characterized by their Schwartz kernels.

Theorem 4.11 ([34, Theorem 4.9]). Given a Green operator $G$ with data as in definition 4.10. Then $G$ has an integral kernel

$$
K_{G} \in\left(C_{\delta, Q}^{\infty}(X) \widehat{\otimes}_{\pi} C_{-\gamma}^{\infty}(X)\right) \cap\left(C_{\delta}^{\infty}(X) \widehat{\otimes}_{\pi} C_{-\gamma, \bar{P}}^{\infty}(X)\right)
$$

where by definition, $C_{\delta, Q}^{\infty}(X)$ is the space of all smooth functions which have asymptotic behavior described by $Q$ when approaching the boundary and $\bar{P}$ is the asymptotic type adjoint to $P$, namely if $P=\left\{\left(p_{j}, m_{j}, L_{j}\right)\right\}$ then $\bar{P}=\left\{\left(\bar{p}_{j}, m_{j}, \bar{L}_{j}\right)\right\}$.

In case $\zeta=\infty$ then

$$
K_{G} \in C_{\delta, Q}^{\infty}(X) \widehat{\otimes}_{\pi} C_{-\gamma, \bar{P}}^{\infty}(X)
$$

Because of this characterization, the mapping properties of Green operators in (4.7) are satisfied for all $1<p<\infty$ provided that it holds for some $p$, that means

$$
\begin{aligned}
& G: \mathcal{H}_{p}^{s, \gamma}(X) \rightarrow \mathcal{H}_{p, P}^{\infty, \delta}(X), \\
& G^{*}: \mathcal{H}_{p^{\prime}}^{s,-\delta}(X) \rightarrow \mathcal{H}_{p^{\prime}, Q}^{\infty,-\gamma}(X) .
\end{aligned}
$$


are continuous.

Moreover, this characterization allows us to change the weight data associated with the prescribed Green operator, as long as we do not interfere with the poles in the asymptotic types.

We fix from now on the notation of weight data $\mathfrak{g}=(\gamma, \gamma-v, \Theta)$ and $\Theta=(-k, 0]$ for some finite number $k$, and we are going to define the cone algebra $C^{\nu}(X, \mathfrak{g})$ of cone pseudo-differential operators of order $v$.

\subsubsection{Mellin pseudo-differential operators}

On manifolds with conic singularities, Mellin transform is also employed to express pseudo-differential operators instead of Fourier transform. The symbols of Mellin pseudodifferential operators turn out to be parameter-dependent pseudo-differential operators on the boundary $Y$.

A Mellin symbol of order $v$ is a symbol $a\left(t, t^{\prime}, z\right) \in C^{\infty}\left(\mathbb{R}_{+} \times \mathbb{R}_{+}, L^{v}\left(Y ; \Gamma_{\frac{1}{2}-\gamma}\right)\right)$ taking values in the space of parameter-dependent pseudo-differential operators $L^{v}\left(Y ; \Gamma_{\frac{1}{2}-\gamma}\right)$. The Mellin pseudo-differential operator corresponding to $a\left(t, t^{\prime}, z\right)$ is determined by the formula

$$
o p_{M}^{\gamma}(h) u(t)=\frac{1}{2 \pi \mathrm{i}} \int_{\Gamma_{\frac{1}{2}-\gamma}} t^{-z} a\left(t, t^{\prime}, z\right)\left\{\int_{0}^{\infty} r^{z-1} u(r) d r\right\} d z
$$

where $u(t) \in C_{0}^{\infty}\left(\mathbb{R}_{+} \times Y\right)$. One understands here the Mellin transform with respect to the variable $\mathrm{t}$, and $a\left(t, t^{\prime}, z\right)$ also acts on the image $\mathcal{M} u(z)$ as on a function on the boundary.

For the calculus of general Mellin pseudo-differential operators, we refer to the book by Egorov-Schulze [11, Section 7.2.3].

Cone-degenerate differential operators in $\operatorname{Diff}_{c}^{v}(X)$ are Mellin pseudo-differential operators with symbols $h(t, z)=\sum_{j=0}^{v} a_{j}(t) z^{j}$, which are differential operators on the boundary of order at most $v$. Moreover, this symbol $h(t, z)$ is an entire function in $z \in C$. It is also natural to consider a special class of symbols, namely holomorphic operator-valued symbols.

Definition 4.12 ([11, 7.2.4, Definition 5]). Let $v \in \mathbb{R}$, the class $M_{O}^{v}(Y)$ of holomorphic Mellin symbols consists of all operator families $h(z)$ which belong to $\mathcal{A}\left(\mathbb{C}, L_{\mathrm{cl}}^{v}(Y)\right)$ and furthermore

$$
h(\beta+\mathrm{i} \tau) \in L_{\mathrm{cl}}^{v}\left(Y ; \Gamma_{\beta}\right)
$$

for all $\beta \in \mathbb{R}$, uniformly in $\left[\beta_{0}, \beta_{1}\right]$, for $-\infty<\beta_{0}<\beta_{1}<+\infty$. 
Besides cone-degenerate differential operators, another example of holomorphic Mellin symbols is the image under the kernel cut-off operator, see [33, 7.2.4, Theorem 2]:

$$
\begin{aligned}
H(\psi): C^{\infty}\left(\overline{\mathbb{R}}_{+} \times \overline{\mathbb{R}}_{+}, L_{\mathrm{cl}}^{v}\left(Y, \Gamma_{0}\right)\right) & \rightarrow C^{\infty}\left(\overline{\mathbb{R}}_{+} \times \overline{\mathbb{R}}_{+}, M_{O}^{v}(Y)\right) \\
a\left(t, t^{\prime}, \beta\right) & \mapsto \mathcal{M}_{\frac{1}{2}, \beta \rightarrow z}\left\{\psi(\beta)\left(\mathcal{M}_{\frac{1}{2}, z \rightarrow \beta}^{-1} a\right)\left(t, t^{\prime}, \beta\right)\right\},
\end{aligned}
$$

where $\psi(\beta) \in C^{\infty}\left(\mathbb{R}_{+}\right)$with $\psi(\beta)=1$ for $|\beta-1| \leq \varepsilon<\frac{1}{2}$.

In cone calculus we also want to find parametrix for an elliptic pseudo-differential operator, in particular, for the one with holomorphic Mellin symbol. Therefore, in the procedure for inverting those symbols, one encounters a class of meromorphic Mellin symbols. To describe the meromorphic structure, one needs the notion of asymptotic type for symbols.

Definition 4.13 ([11, 8.1.2, Definition 1]). A discrete asymptotic type $R$ (for Mellin symbols) is a sequence

$$
P=\left\{\left(r_{j}, n_{j}, N_{j}\right) \mid \mathfrak{R} r_{j} \rightarrow \pm \infty \text { for } j \rightarrow \mp \infty, n_{j} \in \mathbb{Z}_{+}, j \in \mathbb{Z}\right\},
$$

where $N_{j} \subset L^{-\infty}(Y)$ are finite-dimensional subspaces containing finite rank operators.

Definition 4.14 ([11, 8.1.2]). Let $R$ be an asymptotic type for Mellin symbols. The space $\mathcal{M}_{R}^{-\infty}(Y)$ of smooth Mellin symbols consists of all meromorphic functions

$$
h(z) \in \mathcal{A}\left(\mathbb{C} \backslash \pi_{\mathbb{C}} R, L^{-\infty}(Y)\right)
$$

which is meromorphic with poles at $r_{j}$ of multiplicities $n_{j}+1$ and whose Laurent coefficient at $\left(z-r_{j}\right)^{-(k+1)}$ belongs to $N_{j}$ for all $0 \leq k \leq n_{j}$ and $j \in \mathbb{Z}$. Moreover, for any $\pi_{\mathbb{C}} R$-excision function $\chi(z)$ we have

$$
(\chi h)(\beta+\mathrm{i} \tau) \in L^{-\infty}\left(Y ; \mathbb{R}_{\tau}\right)
$$

uniformly in $\left[\beta_{0}, \beta_{1}\right]$, for $-\infty<\beta_{0}<\beta_{1}<+\infty$.

For general order $v \in \mathbb{Z} \cup-\infty$, we set

$$
\mathcal{M}_{R}^{v}(Y)=\mathcal{M}_{O}^{v}(Y)+\mathcal{M}_{R}^{-\infty}(Y)
$$

The space $\mathcal{M}_{P}^{v}(Y)$ then is a Fréchet space with the topology of non-direct sum.

These smoothing Mellin symbols give rise to the class of Mellin smoothing operators. These operators on one hand improve smoothness with respect to the $y$ variables, and on the other hand also create some asymptotic as approaching the boundary. Smoothing Mellin operators and Green operators also form an algebra $C_{M+G}^{v}(X)$ with symbolic structure, for details see [33]. 
Definition 4.15 ([11, Section 8.1.2]). Smoothing Mellin operators are finite linear combinations $M=\sum_{j=0}^{k-1} M_{j}$ of operators of the following form

$$
M_{j}=t^{-v+j} \omega \mathrm{op}_{M}^{\alpha_{j}}\left(h_{j}\right) \widetilde{\omega}
$$

where the symbols $h_{j}(z) \in M_{R_{j}}^{-\infty}(X)$ with certain asymptotic types $R_{j}$ such that $\pi_{\mathbb{C}} R_{j} \cap$ $\Gamma_{\frac{1}{2}-\alpha_{j}}=\emptyset$, and

$$
\gamma+\frac{1}{2}-\frac{N+1}{p}-j \leq \alpha_{j} \leq \gamma+\frac{1}{2}-\frac{N+1}{p}
$$

Remark 4.16. In the case the weight strip is finite $\Theta=(-k, 0], k<\infty$, the operators $A_{j}$ are Green operators, $A_{j} \in C_{G}(X, \mathfrak{g})$ for $j \geq k$.

The weights $\alpha_{j}$ chosen by (4.12) provide continuity of the operators

$$
A_{j}: \mathcal{H}_{p}^{s, \gamma}(X) \rightarrow \mathcal{H}_{p}^{\infty, \gamma-\gamma}(X), j=0,1, \ldots, k-1
$$

Definition 4.17 ([11, 8.1.4, Definition 1]). The cone algebra $C^{v}(X, \mathfrak{g})$ of order $v$ with respect to the weight data $\mathfrak{g}$ is the space of all operators $A$ which is of the following form

$$
A=(1-\omega) \widetilde{A}(1-\tilde{\omega})+\omega t^{-v} \mathrm{op}_{M}^{\gamma-\frac{N}{2}}(h) \tilde{\omega}+M+G,
$$

with symbol $h \in C^{\infty}\left(\overline{\mathbb{R}}_{+}, M_{O}^{v}(Y)\right), \widetilde{A} \in L_{\mathrm{cl}}^{v}($ int $X), M=\sum_{j=0}^{k-1} M_{j}$ with $M_{j}$ are as in (4.11), and $G$ is a Green operator. Here $\omega, \tilde{\omega}$ are arbitrary cut-off functions.

An immediate consequence of the choice of weight (4.12) and the properties of Mellin pseudo-differential operators is the continuity of cone pseudo-differential operators between weighted Sobolev spaces.

Theorem 4.18 ([31, Proposition 2.13]). Each operator $A \in C^{v}(X, \mathfrak{g})$ induces a continuous mapping

$$
A: \mathcal{H}_{p}^{s, \gamma}(X) \rightarrow \mathcal{H}_{p}^{s-\gamma, \gamma-\gamma}(X),
$$

for all $s \in \mathbb{R}$. Moreover, for each asymptotic type $P$, there exists an asymptotic type $Q$ depending on $A$ and $Q$ such that

$$
A: \mathcal{H}_{p, P}^{s, \gamma}(X) \rightarrow \mathcal{H}_{p, Q}^{s-\gamma, \gamma-\gamma}(X)
$$

Remark 4.19. The minimal asymptotic type $Q$ such that the operator $A: \mathcal{H}_{p, P}^{s, \gamma}(X) \rightarrow$ $\mathcal{H}_{p, Q}^{s-\gamma, \gamma-v}(X)$ is continuous is called the push-forward of the asymptotic type $P$ under the action of $A$ and denoted by $P^{A}$. The existence of the minimal asymptotic type was proved in [27]. 
With each cone pseudo-differential operator we associate two principal symbols: the compressed principal symbol which is given in local coordinates by

$$
\sigma_{\psi, b}^{v}(A)(t, y, t \tau, \eta)=\sigma_{\psi}^{v}(\widetilde{A})(t, y, \tau, \eta)
$$

and the principal conormal symbol

$$
\sigma_{M}^{v}(A)(z)=h(0, z)+\sigma_{M}^{v}(M)(z) .
$$

The principal conormal symbol is a meromorphic Mellin symbol with meromorphic structure given by the asymptotic type of the symbol $h_{0}(z)$ of the operator $M$.

In local coordinates, we also define the sequence of conormal symbols of $A$ of lower orders

$$
\sigma_{M}^{\nu-j}(A)(z)=\frac{1}{j !} \frac{\partial^{j} h}{\partial t^{j}}(0, z)+\sigma_{M}^{v-j}(M)(z)
$$

for $j=0,1, \ldots, k-1$.

The symbols of composition of two operators and of the adjoint of an operator are given in the following theorems.

Theorem 4.20 ([11, 8.1.4, Theorem 6]). Let $A \in C^{v}(X, \mathfrak{g}), \mathfrak{g}=(\gamma, \gamma-v, \Theta)$ and $B \in$ $C^{\mu}(X, \tilde{\mathfrak{g}}), \tilde{\mathfrak{g}}=(\gamma-v, \gamma-v-\mu, \Theta)$. Then their composition BA belongs to $C^{\mu+v}(X, \mathfrak{h})$ where $\mathfrak{h}=(\gamma, \gamma-v-\mu, \Theta)$, and its symbols are given by

$$
\begin{aligned}
& \sigma_{\psi}^{\mu+v}(B A)=\sigma_{\psi}^{\mu}(B) \sigma_{\psi}^{\nu}(A), \\
& \sigma_{M}^{\mu+\nu-j}(B A)(z)=\sum_{l+\tilde{l}=j} \sigma_{M}^{\mu-\tilde{l}}(B)(z+v-l) \sigma_{M}^{\nu-l}(A)(z)
\end{aligned}
$$

for $j=0, \ldots, k-1$.

Theorem 4.21 ([11, 8.1.4, Theorem 5]). Given an operator $A \in C^{v}(X, \mathfrak{g}), \mathfrak{g}=(\gamma, \gamma-v, \Theta)$. Then its formal adjoint operator $A^{*}$ with respect to $L^{2}(X, d \mu)$ (with fixed weight $\gamma$ ) belongs to $C^{v}\left(X, \mathfrak{g}^{*}\right)$ where $\mathfrak{g}^{*}=(-\gamma+\nu,-\gamma, \Theta)$, and its symbols are given by

$$
\sigma_{\psi}^{v}\left(A^{*}\right)=\overline{\sigma_{\psi}^{v}(A)}, \quad \sigma_{M}^{v-j}\left(A^{*}\right)(z)=\sigma_{M}^{v-j}(N+1-2 \gamma-\bar{z}-v+j)^{*},
$$

$j=0, \ldots, k-1$, and $*$ on the right-hand side is the formal adjoint in $L^{2}(Y, d y)$.

\subsubsection{Ellipticity and regularity with asymptotics}

Definition 4.22 ([11, 8.1.5, Definition 1]). An operator $A \in C^{v}(X, \mathfrak{g})$ is called cone-elliptic with respect to the weight $\gamma$ if 
(i) the interior principal symbol $\sigma_{\psi}^{v}(A)$ is an isomorphism on $\operatorname{Hom}\left(\pi^{*} E, \pi^{*} F\right), \pi^{*} E, \pi^{*} F$ are induced bundles over $T^{*}$ (int $X$ ), and the compressed principal symbol $\sigma_{\psi, b}^{v}(A)$ is an isomorphism up to $t=0$.

(ii) the operator $\sigma_{M}^{v}(A)(z): H^{s}(Y) \rightarrow H^{s-v}(Y)$ is an isomorphism for at least one $s=$ $s_{0} \in \mathbb{R}$ and for all $z \in \Gamma_{\frac{N+1}{2}-\gamma}$.

It was proved in [31, Section 3.3] that this definition of ellipticity is equivalent to the two conditions below:

1. the interior principal symbol $\sigma_{\psi}^{v}(A)$ is an isomorphism on $\operatorname{Hom}\left(\pi^{*} E, \pi^{*} F\right)$.

2. the conormal symbol $\sigma_{M}^{v}(A)(z) \in L_{\mathrm{cl}}^{v}(Y)$ is invertible for each $z \in \Gamma_{\frac{N+1}{2}-\gamma}$ and $\sigma_{M}^{v}(A)(z)^{-1} \in L_{\mathrm{cl}}^{-v}\left(Y ; \Gamma_{\frac{N+1}{2}-\gamma}\right)$.

This fact and the results on spectral invariance of pseudo-differential operators allow us to replace the invertibility of the conormal symbol in the definition 4.22 , ii) by:

ii') the operator $\sigma_{M}^{v}(A)(z): H_{p}^{s}(Y) \rightarrow H_{p}^{s-v}(Y)$ is an isomorphism for $z \in \Gamma_{\frac{N+1}{2}-\gamma}$ and for any choice of $s \in \mathbb{R}, p>1$.

Elliptic operators admit parametrices in the cone algebra.

Definition 4.23 ([11, 8.1.5, Definition 5]). Given an operator $A \in C^{v}(X, \mathfrak{g})$, then a parametrix for $A$ is an operator $P \in C^{-v}(X,(\gamma-v, \gamma, \Theta))$ such that

$$
P A-I \in C_{G}(X,(\gamma, \gamma, \Theta)), A P-I \in C_{G}(X,(\gamma-v, \gamma-v, \Theta)) .
$$

Theorem 4.24 ([11, 8.1.5, Theorem 7], [31, Corollaries 3.3, 3.4]). Given an operator $A \in C^{v}(X, \mathfrak{g})$, then the following conditions are equivalent

a) $A$ is an elliptic operator with respect to the weight $\gamma_{p}=\frac{N+1}{2}-\frac{N+1}{p}+\gamma$.

b) the operator

$$
A: \mathcal{H}_{p}^{s, \gamma}(X) \rightarrow \mathcal{H}_{p}^{s-\gamma, \gamma-\gamma}(X)
$$

is Fredholm for an $s=s_{0} \in \mathbb{R}$.

If $A$ is elliptic with respect to the weight $\gamma$ then the operator (4.16) is Fredholm for all $s \in$ $\mathbb{R}$. There exists a parametrix P for A. Furthermore, if $A u \in \mathcal{H}_{p}^{s-v, \gamma-v}(X)$ and $u \in \mathcal{H}_{p}^{-\infty, \gamma}(X)$ then $u \in \mathcal{H}_{p}^{s, \gamma}(X)$.

And if

$$
A u \in \mathcal{H}_{p, Q}^{s-v, \gamma-v}(X)
$$

for some asymptotic type $Q$, then $u \in \mathcal{H}_{p, P}^{s, v}(X)$ for a certain type $P$ depending on A and $Q$. 


\subsection{Extensions of cone-degenerate differential operators}

In the present work, we require that $A^{\prime} A: E \rightarrow E^{\prime}$ is elliptic with respect to the weights $\gamma$ and $\gamma+v$. We denote by $A_{\min }$ and $A_{\max }$ the minimal and maximal closed extensions of $A$, where

$$
D\left(A_{\max }\right)=D\left(A_{\max }^{\gamma, p}\right)=\left\{u \in \mathcal{H}_{p}^{0, \gamma}(X ; E) \mid A u \in \mathcal{H}_{p}^{0, \gamma}(X ; F)\right\}
$$

and $D\left(A_{\min }\right)=D\left(A_{\min }^{\gamma, p}\right)$ is the closure of $C_{c}^{\infty}($ int $X ; E)$ with respect to the graph norm

$$
\|u\|=\|u\|_{\mathcal{H}_{p}^{0, \gamma}(X ; E)}+\|A u\|_{\mathcal{H}_{p}^{0, \gamma}(X ; F)} .
$$

In case $A$ is elliptic, these extensions are well understood by Lesch [26] and by GilMendoza[13] in the $L^{2}$ base, and by Seiler-Schrohe [32] in $L^{p}$ base. Generically, the minimal domain is $\mathcal{H}_{p}^{v, \gamma+v}(X ; E)$ and in case $A$ is elliptic, the maximal domain is the sum of the minimal domain with a finite dimensional space, these results are summarized in the appendix.

Under the assumption that $A^{\prime} A$ is elliptic, we can show a similar result, namely

Lemma 4.25. Assume that $A^{\prime} A$ is elliptic with respect to the weights $\gamma$ and $\gamma+v$. Then

$$
D\left(A_{\max }\right)=\mathcal{H}_{p, P}^{v, \gamma}(X ; E), D\left(A_{\min }\right)=\mathcal{H}_{p, O}^{v, \gamma}(X ; E)
$$

for some asymptotic type $P$. Consequently, $D\left(A_{\min }\right)$ has finite codimension in $D\left(A_{\max }\right)$, and both spaces are compactly embedded in $\mathcal{H}_{p}^{0, \gamma}(X ; E)$.

Proof. Given an element $u \in D\left(A_{\max }\right)$, by definition $A u \in \mathcal{H}_{p}^{0, \gamma}(X ; F)$ (here we have identified $F \cong F^{\prime}$ via the bundle metric $h_{F}$ ). Applying $A^{\prime}$ to both sides, it implies that $A^{\prime} A u \in \mathcal{H}_{p}^{-v, \gamma-v}(X ; F)$. Using ellipticity of $A^{\prime} A$ with respect to the weight $\gamma+v$ and the a priori knowledge that $u \in \mathcal{H}_{p}^{0, \gamma}(X ; E)$, we obtain that $u \in \mathcal{H}_{p, P}^{v, \gamma}(X ; E)$ for some asymptotic type $P$ to the weight data $(\gamma-v,(-v, 0])$. This implies that $A u \in \mathcal{H}_{p, P^{A}}^{0, \gamma-v}(X)$. But in fact, we know that

$$
A u \in \mathcal{H}_{p}^{0, \gamma}(X ; F) \subset \mathcal{H}_{p, P^{A}}^{0, \gamma-v}(X ; F) .
$$

Therefore, there exists an asymptotic type $Q \leq P$ such that $u \in \mathcal{H}_{p, Q}^{v, \gamma}(X ; E)$. Hence, we have shown that $D\left(A_{\max }\right)=\mathcal{H}_{p, Q}^{v, \gamma}(X ; E)$. Consequently, the minimal domain is $D\left(A_{\min }\right)=$ $\mathcal{H}_{p, O}^{v, \gamma}(X ; E)$. Combining these facts, we obtain that

$$
D\left(A_{\max }\right) / D\left(A_{\min }\right)=\mathcal{H}_{p, Q}^{v, \gamma}(X ; E) / \mathcal{H}_{p, O}^{v, \gamma}(X ; E) \subset \mathcal{H}_{p, P}^{v, \gamma}(X ; E) / \mathcal{H}_{p, O}^{v, \gamma}(X ; E)
$$


The latter quotient has finite dimension because $A^{\prime} A$ is elliptic, and $P$ is the asymptotic generated by $A^{\prime} A$. We conclude that $\operatorname{dim} D\left(A_{\max }\right) / D\left(A_{\min }\right)<\infty$.

By definition of spaces with asymptotic, $\mathcal{H}_{p, O}^{v, \gamma}(X ; E)$ has some improvement in the order $\gamma$, therefore, $\mathcal{H}_{p, O}^{\gamma, \gamma}(X ; E) \hookrightarrow \hookrightarrow \mathcal{H}_{p}^{0, \gamma}(X ; E)$. We obtain the compact embeddings

$$
D\left(A_{\min }\right) \hookrightarrow D\left(A_{\max }\right) \hookrightarrow \hookrightarrow \mathcal{H}_{p}^{0, \gamma}(X ; E)
$$

We are now aiming at showing a Poincaré-type inequality, which holds for functions belonging to certain subspace of the maximal domain of the operator $A$. First, we check that $A$ is a semi-Fredholm operator acting between $D\left(A_{\max }\right)$ and $\mathcal{H}_{p}^{0, \gamma}(X ; F)$. We need the following criterion for semi-Fredholm property.

Lemma 4.26 ([30, Lemma 3]). Let $X, Y, Z$ be reflexive Banach spaces, and $Y$ be compactly embedded in $X$. Assume that $T: Y \rightarrow Z$ is a continuous linear map. Then the following are equivalent:

1. $T$ is an upper semi-Fredholm operator, which means that $T$ has closed range and its nullspace is finite-dimensional,

2. for all $u \in Y$, it holds

$$
\|u\|_{Y} \leq C\left(\|u\|_{X}+\|T u\|_{Z}\right) .
$$

Corollary 4.27. Consider a cone-differential operator $A: D\left(A_{\max }\right) \rightarrow \mathcal{H}_{p}^{0, \gamma}(X ; E)$. Then $A$ is a semi-Fredholm operator. In particular, Ker $A$ has finite dimension in $D\left(A_{\max }\right)$.

Furthermore, denote by $V$ an arbitrary complement of $\operatorname{Ker} A$ in $\mathcal{H}_{p}^{0, \gamma}(X ; E)$, for all $v \in V$, it holds

$$
\|v\|_{V} \leq C\|A u\|_{\mathcal{H}_{p}^{0, \gamma}(X ; F)} .
$$

Proof. We apply Lemma 4.26 to the case

$$
X=\mathcal{H}_{p}^{0, \gamma}(X ; E), Y=D\left(A_{\max }\right) \text {, and } Z=\mathcal{H}_{p}^{0, \gamma}(X ; F) \text {. }
$$

We have proved in Lemma 4.25 that $Y$ is compactly embedded in $X$. Moreover, $Y$ is furnished with the graph norm

$$
\|u\|_{Y}=\|u\|_{\mathcal{H}_{p}^{0, \gamma}(X ; E)}+\|A u\|_{\mathcal{H}_{p}^{0, \gamma}(X ; F)}=\|u\|_{X}+\|A u\|_{Z} .
$$


It means condition 4.17) holds for $C=1$. Therefore, Lemma 4.26 implies that $\operatorname{Ker} A \cap$ $D\left(A_{\max }\right)$ has finite dimension.

If $V$ is an arbitrary complement of $\operatorname{Ker} A$ in $D\left(A_{\max }\right)$, then the first isomorphism theorem tells us that $V$ and $\operatorname{Im} A$ are homeomorphic, in other words, for all $v \in V$

$$
C_{1}\left|\|A u \mid\|_{\mathcal{H}_{p}^{0, \gamma}} \leq\|v\|_{V} \leq C_{2}\|A u\|_{\mathcal{H}_{p}^{0, \gamma}}\right.
$$

We also give a proof of the Poincaré-type inequality using functional tools for a space $D$ such that $D \cap \operatorname{Ker} A=\{0\}$.

Proposition 4.28 (Poincaré type inequality). Assume that $D$ is a closed subspace of $\mathcal{H}_{p}^{0, \gamma}(X)$ such that $D \cap \operatorname{Ker} A=\{0\}$. Then the following inequality holds

$$
\|u\|_{\mathcal{H}_{p}^{0, \gamma}(X)} \leq C\|A u\|_{\mathcal{H}_{p}^{0, \gamma}(X)}
$$

for all $u \in D$.

Proof. Set

$$
\inf _{u \in \mathcal{E}}\|A u\|_{\mathcal{H}_{p}^{0, \gamma}(X)}=\alpha,
$$

where $\mathcal{E}=\left\{u \in D,\|u\|_{\mathcal{H}_{p}^{0, \gamma}(X)}=1\right\}$. We claim that $\alpha>0$.

Indeed, assume that $\left\{u_{m}\right\} \subset \mathcal{E}$ is a minimizing sequence of $\|A u\|_{\mathcal{H}_{p}^{0, \gamma}(X)}$. Because $\left\{u_{m}\right\} \subset$ $\mathcal{E}$ is a bounded sequence in $D$, there exists a subsequence (we use the same notation) which converges weakly in to an element $u \in D$. Because $D$ is compactly embedded in $\mathcal{H}^{0, \gamma}(X)$. Therefore, $u_{m}$ converges strongly to $u$ in $\mathcal{H}_{p}^{0, \gamma}(X)$. Strong convergence yields that $\|u\|_{\mathcal{H}_{p}^{0, \gamma}(X)}=1, u \in \mathcal{E}$. And weak convergence in $D$ gives us that

$$
\|A u\|_{\mathcal{H}_{p}^{0, \gamma}(X)} \leq \lim _{m \rightarrow \infty}\left\|A u_{m}\right\|_{\mathcal{H}_{p}^{0, \gamma}(X)}=\alpha
$$

and thus, we obtain $\|A u\|_{\mathcal{H}_{p}^{0, \gamma}(X)}=\alpha$.

Moreover, using $D \cap \operatorname{Ker} A=\{0\}$, we conclude that $\alpha=\|A u\|_{\mathcal{H}_{p}^{0, \gamma}(X)}>0$. And the inequality holds for $C=\alpha^{-1}$. Furthermore, one obtains

$$
\|u\|=\|u\|_{\mathcal{H}_{p}^{0, \gamma}(X)}+\|A u\|_{\mathcal{H}_{p}^{0, \gamma}(X)} \sim\|A u\|_{\mathcal{H}_{p}^{0, \gamma}(X)}, \forall u \in D
$$




\subsection{Existence of finite energy solutions}

Throughout this chapter, we will be concerned about higher-order equations of the following form

$$
A^{\prime} \mathcal{F}(x, A u)+\mathcal{G}(x, B u)=f, x \in X \backslash Y,
$$

where $A \in \operatorname{Diff}_{c}^{v}(X)$ is a cone-degenerate differential operator, some weight $\gamma \in \mathbb{R}$ is fixed and $A^{\prime}$ is the transpose of $A$ with respect to $L^{2}(X, d \mu)$. The measure has the expression $d \mu=t^{N-\gamma p} d t d y$ in local coordinates.

Now we fix a domain $D\left(A_{\min }\right) \subset D \subset D\left(A_{\max }\right)$ which corresponds to specified cone conditions. As $D\left(A_{\max }\right) / D\left(A_{\min }\right)$ has finite dimension, $D$ is closed in $D\left(A_{\max }\right)$, and both are compactly embedded in $\mathcal{H}_{p}^{0, \gamma}(X ; E)$. Moreover, $D$ is a reflexive space.

We state here without proof a Nemytskii type theorem adapted to weighted $L^{p}$ spaces. We use this proposition to obtain the continuity property of nonlinear operators.

Proposition 4.29 ([9, Theorem 1.1]). Let $\Omega$ be a measurable set of positive measure in $\mathbb{R}^{n}$. Given a real-valued function $h\left(x, u_{1}, u_{2}, \ldots, u_{N}\right): \Omega \times \mathbb{R}^{N} \rightarrow \mathbb{R}$ satisfying the following conditions:

a) $h\left(x, u_{1}, u_{2}, \ldots, u_{N}\right)$ is a continuous function with respect to $u_{1}, \ldots, u_{N}$ for almost all $x \in \Omega$,

b) $h\left(x, u_{1}, u_{2}, \ldots, u_{N}\right)$ is a measurable function in $x$ for any fixed $u_{1}, \ldots, u_{N}$,

c) the inequality

$$
\left|h\left(x, u_{1}, u_{2}, \ldots, u_{N}\right)\right| \leq C \omega_{0}^{-1 / p} \sum_{i=1}^{N} \omega_{i}^{1 / p}\left|u_{i}\right|^{p_{i} / p}+\omega_{0}^{-1 / p} g(x)
$$

holds for a positive constant $C$, some numbers $p, p_{1}, \ldots, p_{N}$ belonging to the interval $(1, \infty)$ and a function $g(x) \in L^{p}(\Omega)$.

Then the Nemytskii operator defined by the equality

$$
H\left[u_{1}(x), \ldots, u_{N}(x)\right]:=h\left(x, u_{1}(x), \ldots, u_{N}(x)\right)
$$

acts continuously from $L^{p_{1}}\left(\omega_{1} d x\right) \times \ldots \times L^{p_{N}}\left(\omega_{N} d x\right)$ to $L^{p}\left(\omega_{0} d x\right)$.

Suppose that the nonlinearities $\mathcal{F}(x, q): X \times E \rightarrow F$ and $\mathcal{G}(x, z): X \times G \rightarrow E$ are continuous and subject to the following conditions for almost $x \in X \backslash Y, q \in E_{x}, z \in G_{x}$ : 
(F1) $\|\mathcal{F}(x, q)\|_{F} \leq h_{1}(x)+|q|^{p-1}$, where $h_{1}(x) \in \mathcal{H}_{p^{\prime}}^{0,0}(X)$.

(F2) $h_{F}(\mathcal{F}(x, q), q) \geq C_{0}\|q\|_{F}^{p}-C_{1}$.

(F3) $h_{F}\left(\mathcal{F}\left(x, q_{1}\right)-\mathcal{F}\left(x, q_{2}\right), q_{1}-q_{2}\right)>0$ as $q_{1}, q_{2} \in F_{x}, q_{1} \neq q_{2}$.

(G1) $h_{E}(G(x, z), z) \leq h_{2}(x)+\|z\|_{G}^{p-1-\varepsilon}$, where $h_{2}(x) \in \mathcal{H}_{p^{\prime}}^{0,0}(X), \varepsilon \geq 0$.

Because of the growth of the nonlinearities, in this section we work with $\gamma=0$ and the corresponding minimal and maximal domains. So in our setting, $D\left(A_{\min }\right) \subset D \subset D\left(A_{\max }\right)$.

Definition 4.30. A weak solution of the equation (4.20) is a function $u \in D$ satisfying the identity follows

$$
\int_{X} h_{F}(\mathcal{F}(x, A u), A v) d \mu=\int_{X} h_{E}(\mathcal{G}(x, B u), v) d \mu \quad \text { for all } v \in D
$$

Let us define the following operators $\mathfrak{F}, \mathfrak{5}: D \rightarrow D^{\prime}$ by the formulae

$$
\begin{aligned}
& \langle\mathfrak{F} u, v\rangle=\int_{X} h_{F}(\mathcal{F}(x, A u), A v) d \mu, \\
& \langle\mathfrak{G} u, v\rangle=\int_{X} h_{E}(\mathcal{G}(x, B u), v) d \mu .
\end{aligned}
$$

Lemma 4.31. Assume that the nonlinearities $\mathcal{F}$ and $\mathcal{G}$ subject to the growth conditions $(F 1),(F 2),(F 3)$ and (G1). We suppose further that either the mapping $B: D \rightarrow \mathcal{H}_{p}^{0,0}(X, E)$ is compact or it is continuous and $\varepsilon>0$.

Then the operators $\mathfrak{F}, \mathfrak{5}: D \rightarrow D^{\prime}$ are well-defined, bounded and continuous. Moreover, $(55$ is compact.

Proof. These operators are well-defined. Indeed, the metric bundle $h_{F}: F \times F \rightarrow X \times \mathbb{R}$ considered as a bilinear mapping in each fiber has a norm depending continuously in $x$. Moreover, $X$ is a compact manifold, there will be a common bound $C$ such that for all $q_{i} \in F_{x}, x \in X$

$$
h_{F}\left(q_{1}, q_{2}\right) \leq C\left\|q_{1}\right\|_{F}\left\|q_{2}\right\|_{F} .
$$


So given an element $u \in D, v \in C_{c}^{\infty}(X \backslash)$, we can estimate as follows

$$
\begin{aligned}
|\langle\mathfrak{F} u, v\rangle| & =\int_{X} h_{F}(\mathcal{F}(x, A u), A v) d \mu \\
& \leq C \int_{X}\|\mathcal{F}(x, A u(x))\|_{F}\|A v(x)\|_{F} d \mu \\
& \leq C \int_{X}\left(|h(x)|+\|A u\|_{F}^{p-1}\right)\|A v\|_{F} d \mu, \quad \text { use }(\mathrm{F} 1) \\
& \leq C\left(\int_{X}\left(|h(x)|^{p^{\prime}}+\|A u(x)\|_{F}^{p}\right) d \mu\right)^{\frac{1}{p^{\prime}}}\left(\int_{X}\|A v(x)\|_{F}^{p} d \mu\right)^{\frac{1}{p}} \\
& \leq C\left(\|u\|_{D}\right)\|v\|_{D} .
\end{aligned}
$$

This inequality means that $\mathfrak{F} u \in D^{\prime}$ for all $u \in D$. Moreover, it also yields that

$$
\|\mathfrak{F} u\|_{D^{\prime}} \leq C\|u\|_{D}
$$

where $C$ depends on the bound of $h_{F}$ in $X$ and the norm $\left\|h_{1}\right\|_{\mathcal{H}_{p^{\prime}}^{0,0}}(X ; \mathbb{R})$. Hence $\mathfrak{F}$ is a bounded operator. The continuity follows by proposition 4.29

We prove similarly for $\mathfrak{b}$.

$$
\begin{aligned}
\mid\langle(\mathfrak{\xi} u, v\rangle| & =\int_{X} h_{E}(\mathcal{G}(x, B u), v) d \mu \\
& \leq \int_{X} C(x)\|\mathcal{G}(x, B u(x))\|_{E}\|v(x)\|_{E} d \mu \\
& \leq C \int_{X}\left(h_{2}(x)+\|B u(x)\|_{E}^{p-1}\right)\|v(x)\|_{E} d \mu \\
& \leq C\left(\left\|h_{2}\right\|_{\mathcal{H}_{p^{\prime}}^{0,0}}+\|B u\|_{\mathcal{H}_{p}^{0,0}(X ; E)}^{p-1}\right)\|v\|_{\mathcal{H}_{p}^{0,0}(X ; E)} \\
& \leq C\left(\left\|h_{2}\right\|_{\mathcal{H}_{p^{\prime}}^{0,0}}+\|u\|_{D}^{p-1}\right)\|v\|_{\mathcal{H}_{p}^{0,0}(X ; E)} \\
& \leq C\left(\|u\|_{D}\right)\|v\|_{D},
\end{aligned}
$$

where in the previous line, we have used the continuity of $B: D \rightarrow \mathcal{H}_{p}^{0,0}(X ; E)$. We also obtain that $5 \mathfrak{5}$ is well-defined, bounded and continuous operator.

To check that $5: D \rightarrow D^{\prime}$ is a compact operator, we combine proposition 4.29 and the property of $B$. When $\varepsilon \geq 0$ and $B: D \rightarrow \mathcal{H}_{p}^{0,0}(X ; E)$ is compact, taking an arbitrary bounded sequence $u_{n}$ in $D$, we will show that $\left(5 u_{n}\right.$ has a convergent subsequence. Indeed, because $u_{n}$ is bounded in the reflexive space $D$, it has a subsequence, also denoted by $u_{n}$, which converges weakly to some $u_{0}$ in $D$. Hence, the sequence $B u_{n}$ converges in $\mathcal{H}_{p}^{0,0}(X, E)$. Applying proposition 4.29 , we obtain that $\left(5 u_{n}\right.$ converges to $5 u_{0}$ in $D^{\prime}$. So 65 is compact. 
When $\varepsilon>0$ and assuming only continuity of $B$, applying proposition 4.29 , we know that the operator

$$
\mathcal{G}(z(\cdot)): \mathcal{H}_{p-\varepsilon}^{0,0}(X ; G) \rightarrow \mathcal{H}_{p}^{0,0}(X ; E)
$$

is continuous. We repeat the proof above, the bounded sequence $\left\{u_{n}\right\}$ is mapped to a bounded sequence $\left\{B u_{n}\right\}$. Therefore, there is a subsequence, which is also indexed by $n$, $B u_{n}$ converging weakly to $B u$ in $\mathcal{H}_{p}^{0,0}(X ; G)$. The compact embedding

$$
\mathcal{H}_{p}^{0,0}(X ; G) \hookrightarrow \hookrightarrow \mathcal{H}_{p-\varepsilon}^{0,0}(X ; G)
$$

implies that $B u_{n} \rightarrow B u$ in $\mathcal{H}_{p-\varepsilon}^{0,0}(X ; G)$ and hence, $\left(5 u_{n}\right.$ converges to $\mathfrak{5} u$ in $\mathcal{H}_{p}^{0,0}(X ; E)$.

In the next part, we will check that $\mathfrak{F}$ has property $\alpha(D)$.

Lemma 4.32. The operator $\mathfrak{F}: D \rightarrow D^{\prime}$ has property $\alpha(D)$.

Proof. We prove similarly the proof in the second-order case.

Indeed, given an arbitrary sequence $u_{n} \rightarrow u$ in $D$ and assume that

$$
\limsup _{n \rightarrow \infty}\left\langle\mathfrak{F} u_{n}, u_{n}-u\right\rangle \leq 0
$$

we need to verify that $u_{n} \rightarrow u$ in $D$.

By the compact embedding $D \hookrightarrow \hookrightarrow \mathcal{H}_{p}^{0,0}(X)$, we have $u_{n} \rightarrow u$ in $\mathcal{H}_{p}^{0,0}(X)$. It remains to show that $A u_{n} \rightarrow A u$ in $\mathcal{H}_{p}^{0,0}(X)$.

Given a measurable subset $E \subset X$, let us set

$$
\lambda_{n}(E)=\int_{E} h_{F}\left(\mathcal{F}\left(x, A u_{n}\right)-\mathcal{F}(x, A u), A u_{n}-A u\right) d \mu .
$$

Because $\mathcal{F}$ is monotone with respect to the second component, see $(\mathrm{F} 3)$, the integrand is nonnegative. Therefore,

$$
0 \leq \lambda_{n}(E) \leq \lambda_{n}(X)
$$

Moreover,

$$
\limsup _{n \rightarrow \infty} \lambda_{n}(X)=\limsup _{n \rightarrow \infty}\left\langle\mathfrak{F} u_{n}-\mathfrak{F} u, u_{n}-u\right\rangle \leq 0
$$

This yields

$$
\lim _{n \rightarrow \infty} \lambda_{n}(X)=0, \text { and } \lim _{n \rightarrow \infty} \lambda_{n}(E)=0
$$

for all $d \mu$-measurable subset $E \subset X$. 
Using conditions (F1) and (F2), we can show

$$
\begin{aligned}
\lambda_{n}(E)= & \int_{E} h_{F}\left(\mathcal{F}\left(x, A u_{n}\right)-\mathcal{F}(x, A u), A u_{n}-A u\right) d \mu \\
= & \int_{E}\left[h_{F}\left(\mathcal{F}\left(x, A u_{n}\right), A u_{n}\right)-h_{F}\left(\mathcal{F}\left(x, A u_{n}\right), A u\right)+h_{F}\left(\mathcal{F}(x, A u), A u-A u_{n}\right)\right] d \mu \\
\geq & \int_{E}\left(C_{0}\left\|A u_{n}(x)\right\|_{F}^{p}-C_{1}\right) d \mu-\int_{E}\left(h_{1}(x)+\left\|A u_{n}(x)\right\|_{F}^{p-1}\right)\|A u(x)\|_{F} d \mu \\
& -\int_{E}\left(h_{1}(x)+\|A u(x)\|_{F}^{p-1}\right)\left(\|A u(x)\|_{F}+\left\|A u_{n}(x)\right\|_{F}\right) d \mu
\end{aligned}
$$

Putting negative terms to the left-hand side, one obtains

$$
\begin{array}{r}
C_{0} \int_{E}\left\|A u_{n}(x)\right\|_{F}^{p} d \mu \leq C_{1} \mu(E)+\lambda_{n}(E)+\int_{E}\left(h_{1}(x)+\|A u(x)\|_{F}^{p-1}\right)\left(\|A u(x)\|_{F}+\left\|A u_{n}(x)\right\|\right) d \mu \\
+\int_{E}\left(h_{1}(x)+\left\|A u_{n}(x)\right\|_{F}^{p-1}\right)\|A u(x)\|_{F} d \mu .
\end{array}
$$

Using Young's inequality we can estimate

$$
\begin{aligned}
\int_{E}\left(h_{1}(x)+\|A u(x)\|_{F}^{p-1}\right)\left(\|A u(x)\|_{F}+\left\|A u_{n}(x)\right\|_{F}\right) d \mu \leq & \left.\int_{E}\|A u(x)\|_{F}^{p}\right) d \mu+\frac{\varepsilon^{p}}{p} \int_{E}\left\|A u_{n}(x)\right\|_{F}^{p} d \mu \\
& +\frac{\varepsilon^{-p^{\prime}}}{p^{\prime}} \int_{E}\left(\left|h_{1}(x)\right|^{p^{\prime}}+\|A u(x)\|_{F}^{p}\right) d \mu \\
\int_{E}\left(h_{1}(x)+\left\|A u_{n}(x)\right\|_{F}^{p-1}\right)\|A u(x)\|_{F} d \mu \leq & \frac{\varepsilon^{-p}}{p} \int_{E}\|A u(x)\|_{F}^{p} d \mu+ \\
& +\frac{\varepsilon^{p^{\prime}}}{p^{\prime}} \int_{E}\left(\left|h_{1}(x)\right|^{p^{\prime}}+\left\|A u_{n}(x)\right\|_{F}^{p}\right) d \mu
\end{aligned}
$$

By choosing appropriate $\varepsilon>0$, we obtain

$$
\int_{E}\left\|A u_{n}(x)\right\|_{F}^{p} d \mu \leq C \int_{E}\left(h_{1}(x)^{p^{\prime}}+\|A u(x)\|_{F}^{p}\right) d \mu+\lambda_{n}(E)+\mu(E) .
$$

Therefore, $\int_{E}\left\|A u_{n}(x)\right\|_{F}^{p} d \mu \rightarrow 0$ as meas $E \rightarrow 0$ uniformly in $n$.

Now we will show that $A u_{n}$ converges to $A u$ in measure. For $\varepsilon, \delta>0$, set

$$
F_{\varepsilon, n}=\left\{x \in X \mid\left\|A u_{n}(x)-A u(x)\right\|_{F} \geq \varepsilon\right\} .
$$

Choose two sets $E_{\delta}^{(1)}$ and $E_{\delta}^{(2)}$ such that

$$
\operatorname{meas}\left(E_{\delta}^{(j)}\right)<\frac{\delta}{4}
$$

$E_{\delta}^{(1)}$ is selected from the condition

$$
K_{\delta}:=\sup _{X \backslash E_{\delta}^{(1)}}\left\{\|A u(x)\|_{F}+\left\|A u_{n}(x)\right\|_{F}\right\}<\infty .
$$


$E_{\delta}^{(2)}$ is selected from the condition

$$
k_{\varepsilon}:=\inf \left\{h_{F}\left(\mathcal{F}(x, q)-\mathcal{F}\left(x, q^{\prime}\right), q-q^{\prime}\right)\right\}>0
$$

where the infimum is taken over the set

$$
\left\{x \in X \backslash E_{\delta}^{(2)},\left|q^{\prime}\right| \leq K,\left|q-q^{\prime}\right| \geq \varepsilon\right\} .
$$

From the definition of the sets $E_{\delta}^{(j)}$, we have

$$
\begin{aligned}
\lambda_{n}(X) & \geq \lambda_{n}\left(F_{\varepsilon, n} \backslash\left(\cup E_{\delta}^{(j)}\right)\right) \\
& \geq k_{\varepsilon}\left(\operatorname{meas} F_{\varepsilon, n}-\operatorname{meas} E_{\delta}^{(1)}-\operatorname{meas} E_{\delta}^{(2)}\right) .
\end{aligned}
$$

Hence meas $F_{\varepsilon, n} \leq \delta / 2+\lambda_{n}(X) / k_{\varepsilon}$, and for large enough $n$, meas $F_{\varepsilon, n}<\delta$.

Since $\mu(X)>0$, the equi-integrability and convergence in measure imply strong convergence of the sequence $A u_{n}$ to $A u$ in $\mathcal{H}_{p}^{0,0}(X)$ and hence, convergence of $u_{n}$ to $u$ in $D$.

Combining the facts in lemma 4.31 and lemma 4.32, we conclude that the operator $\mathfrak{F}+(\mathfrak{5}$ also satisfies property $\alpha(D)$. We will make another assumption, which guarantees that the mapping degree of $\operatorname{Deg}(\mathfrak{F}+(\mathfrak{5}, \overline{B(0, R)}, f)$ can be well-defined in certain subset $B(0, R) \subset D$.

We require a stronger growth condition on the nonlinearities

$$
\text { (C) }: h_{F}(\mathcal{F}(x, q), q)+h_{E}(\mathcal{G}(x, \xi), u) \geq C_{0}\|q\|_{F}^{p}+C_{2}\|u\|_{E}^{p} \text {. }
$$

This condition guarantees coercivity of $\mathfrak{F}+\mathfrak{G}$. Indeed,

$$
\begin{aligned}
\langle\mathfrak{F} u+\mathfrak{5} u, u\rangle & =\int_{X}\left[h_{F}(\mathcal{F}(x, A u), A u)+h_{E}(\mathcal{G}(x, B u), u)\right] d \mu \\
& \geq \int_{X}\left[C_{0}\|A u(x)\|_{F}^{p}+C_{2}\|u\|_{E}^{p}\right] d \mu \\
& \geq C\|u\|_{D}^{p} \\
\Rightarrow & \lim _{\|u\|_{D} \rightarrow \infty} \frac{\langle\mathfrak{F} u+\mathfrak{5} u, u\rangle}{\|u\|_{D}}=\infty \text { as } p>1 .
\end{aligned}
$$

We proceed as in the proof of theorem 3.3 . There exists a ball $B(0, R)$ for sufficiently large $R>0$, such that we can assign the mapping degree to $\mathfrak{F}+(\mathfrak{5}$ on the set $\overline{B(0, R) \cap D}$ with respect to $f$. Moreover, $\operatorname{Deg}(\mathfrak{F}+(\mathfrak{F}, \overline{B(0, R) \cap D}, f)=1$. We conclude that the equation $(\mathfrak{F}+(\mathfrak{5}) u=f$ possesses at least one solution.

We have proved the following theorem. 
Theorem 4.33. Assume that the nonlinearities $\mathcal{F}: X \times E \rightarrow F$ and $\mathcal{G}: X \times G \rightarrow E$ are continuous and subject to growth conditions $(F 1),(F 2),(F 3),(G 1)$, and the coercive condition $(C)$. Suppose further that either $B: D \rightarrow \mathcal{H}_{p}^{0,0}(X ; E)$ is compact or it is continuous and $\varepsilon>0$.

Then for all $f \in D^{\prime}$, there exists at least one solution to the equation 4.20 .

Remark 4.34. In the case the domain $D$ satisfying $\operatorname{Ker} A \cap D=\{0\}$, then the Poincaré-type inequality 4.28 holds, consequently $\|u\|_{D} \sim\|A u\|_{\mathcal{H}_{p}^{0,0}}$, we can relax the coercivity condition (C) by supposing that

$$
h_{F}(\mathcal{F}(x, q), q)+h_{E}(\mathcal{G}(x, \xi), u) \geq C\|q\|_{F}^{p}
$$

for all $x \in X, \xi \in G_{x}$, and $q \in F_{x}$.

\subsection{Existence of singular solutions}

In this part, we will work with quasilinear elliptic equations of the following form

$$
A^{\prime} \mathcal{F}(x, A u)+C^{\prime} \mathcal{G}(x, B u)=f \quad \text { in } X \backslash Y .
$$

Let us recall that we have fixed a weight $\gamma \in \mathbb{R}$ and $A^{\prime}$ and $C^{\prime}$ are the formal adjoint operators of $A$ and $C$ respectively with respect to the measure $d \mu$ which has form $d \mu=t^{N-\gamma p} d t d y$ in local coordinates in a collar neighborhood of the boundary. The cone-degenerate operators $A \in \operatorname{Diff}_{c}^{v}(X ; E, F), B \in \operatorname{Diff}_{c}^{\nu_{0}}\left(X ; E, G_{0}\right), C \in \operatorname{Diff}_{c}^{\nu_{1}}\left(X ; E, G_{1}\right)$ are of indicated order, and $v_{0}+v_{1}<2 v$.

Let us suppose that the nonlinearity $\mathcal{F}(x, q): X \times F \rightarrow F$ is differentiable in the variables $q$ and measurable in the first component. It subjects to further conditions as follows for all $x \in X \backslash Y$ and $q_{1}, q_{2} \in F_{x}$ :

$$
h_{F}\left(\mathcal{F}\left(x, q_{1}\right)-\mathcal{F}\left(x, q_{2}\right), q_{1}-q_{2}\right) \geq C\left\|q_{1}-q_{2}\right\|_{F}^{2}\left(\left\|q_{1}\right\|_{F}^{p-2}+\left\|q_{2}\right\|_{F}^{p-2}\right)
$$

and

$$
\left\|\mathcal{F}\left(x, q_{1}\right)-\mathcal{F}\left(x, q_{2}\right)\right\|_{F} \leq\left\|q_{1}-q_{2}\right\|_{F}\left(\left\|q_{1}\right\|_{F}^{p-2}+\left\|q_{2}\right\|_{F}^{p-2}\right)
$$

Furthermore, assume that $\mathcal{F}$ is homogeneous in the second variable, namely, for $(x, q) \in F$ and a number $\lambda>0$ then

$$
\mathcal{F}(x, \lambda q)=\lambda|\lambda|^{p-2} \mathcal{F}(x, q) \text { in } F_{x} .
$$


We are looking for a singular solution to (4.22), provided that there exists a formal solution of the form

$$
e(t, y) \sim t^{-\lambda_{0}} \varphi(y) \text { as }(t, y) \rightarrow Y,
$$

with an exponent $\lambda_{0}>0$ and $\varphi(y) \in C^{\infty}(Y)$. This assumption is equivalent to the solvability of the nonlinear eigenvalue problem corresponding to 4.22 .

Lemma 4.35. The asymptotic data $\left(\lambda_{0}, \varphi(y)\right)$ is a solution of the following nonlinear eigenvalue problem

$$
\sigma_{M}^{v}\left(A^{\prime}\right)\left(\left(\lambda_{0}+v\right)(p-1)\right) \mathcal{F}\left(\sigma_{M}^{v}(A)\left(\lambda_{0}\right) \varphi(y)\right)=0
$$

Proof. This lemma is proved by calculating formally and forcing the leading asymptotic term to vanish. Indeed, $e(t, y) \sim t^{-\lambda_{0}} \varphi(y)$ gives us

$$
A e \sim t^{-\lambda_{0}-v} \psi(y), \text { where } \psi(y)=\sigma_{M}^{v}(A)\left(\lambda_{0}\right) \varphi(y) .
$$

The homogeneity of $\mathcal{F}$ implies that

$$
\mathcal{F}(x, A e) \sim t^{-\left(\lambda_{0}+v\right)(p-1)} \mathcal{F}(x, \psi(y)) .
$$

Applying the operator $A^{\prime}$ to both sides, we obtain

$$
A^{\prime} \mathcal{F}(x, A e) \sim t^{-\left(\lambda_{0}+v\right)(p-1)-v} \sigma_{M}^{v}\left(A^{\prime}\right)\left(\left(\lambda_{0}+v\right)(p-1)\right) \mathcal{F}\left(\sigma_{M}^{v}(A)\left(\lambda_{0}\right) \varphi(y)\right) .
$$

The leading order term on the left-hand side necessarily vanishes, that implies that $\left(\lambda_{0}, \varphi(y)\right)$ is a solution of the nonlinear eigenvalue problem 4.26 ).

Let us denote

$$
L_{0}=\sigma_{M}^{v}(A)\left(\lambda_{0}\right), L_{1}=\sigma_{M}^{v}\left(A^{\prime}\right)\left(\left(\lambda_{0}+v\right)(p-1)\right)
$$

Both are differential operators of order $v$ on the boundary $Y$. We can rewrite $L_{1}$ as

$$
L_{1}=\sigma_{M}^{v}(A)\left(N+1-2 \gamma-\left(\lambda_{0}+v\right)(p-1)-v\right)^{*},
$$

where the $*$ denotes the adjoint operator with respect to $L^{2}(Y)$.

Let us verify now that $L_{0}-L_{1}^{*}$ is a compact operator on the boundary $Y$ by applying the formular 2.5) to $L_{0}$ and $L_{1}^{*}$. We have

$$
\begin{aligned}
& \sigma_{\psi}^{v}\left(L_{0}\right)(y, \eta)=\widetilde{\sigma}_{\psi}^{v}(A)\left(0, y,-\mathrm{i} \lambda_{0}, \eta\right) \\
& \sigma_{\psi}^{v}\left(L_{1}^{*}\right)(y, \eta)=\widetilde{\sigma}_{\psi}^{v}(A)\left(0, y, \mathrm{i}\left(\left(\lambda_{0}+v\right)(p-1)+v+2 \gamma-N-1\right), \eta\right) .
\end{aligned}
$$


The polynomials $\sigma_{\psi}^{v}\left(L_{0}\right)(y, \eta)$ and $\sigma_{\psi}^{v}\left(L_{1}^{*}\right)(y, \eta)$ in $\eta$ have the same highest order, namely $\sigma_{\psi}^{v}\left(a_{k}(0)\right)(y, \eta)$, therefore $\sigma_{\psi}^{v}\left(L_{1}^{*}\right)(y, \eta)-\sigma_{\psi}^{v}\left(L_{0}\right)(y, \eta)$ is a polynomial of order at most $v-1$. It means that $L_{1}^{*}-L_{0}$ is a compact operator acting between Sobolev spaces on $Y$.

We can rewrite the equation 4.26 as

$$
L_{0}^{*} \mathcal{F}\left(L_{0} \varphi(y)\right)+\text { compact perturbation }=0
$$

Under certain conditions of the compact perturbation, this equation has a solution $\varphi(y)$. In this work let us assume that:

Assumption: There exist solutions $\left(\lambda_{0}, \varphi(y)\right)$ to the equation 4.26).

Let us pick a solution $\left(\lambda_{0}, \varphi(y)\right)$ of the problem 4.26$), \lambda_{0}>0$, that is $e(t, y) \sim t^{-\lambda_{0}} \varphi(y)$ is a singular formal solution. Further asymptotic terms in $e(t, y)$ can be calculated in principle by formal asymptotic analysis. We make a requirement that $e(t, y)$ is manipulated until a remainder part which belongs to a space of certain smoothness, which we will define now.

Let us denote $\mathfrak{B}$ the space of all functions $u \in \mathcal{H}_{p}^{0, \gamma}(X ; E) \cap \mathcal{H}^{0, \rho}(X ; E)$ such that $A u \in \mathcal{H}_{p}^{0, \gamma}(X ; F) \cap \mathcal{H}^{0, \rho}(X ; F)$, where $\rho=\frac{(p-2)\left(\lambda_{0}+v\right)}{2}+\frac{p \gamma}{2}$. The metric in the affine space $e+\mathfrak{W}$ is induced by the metric in $\mathfrak{B}$.

We reuse the notations $\|v\|_{1},\|v\|_{2}$ for the seminorms in $\mathfrak{B}$, where

$$
\|v\|_{1}=\left(\int_{X}\|A v(x)\|_{F}^{2}\|A e(x)\|_{F}^{p-2} d \mu\right)^{\frac{1}{2}},\|v\|_{2}=\left(\int_{X}\|A v(x)\|^{p} d \mu\right)^{\frac{1}{p}}=\|A v\|_{\mathcal{H}_{p}^{0, \gamma}(X ; F)} .
$$

The choice of $\gamma$ gives us the equivalence $\|A v\|_{\mathcal{H}^{0, \rho}} \sim\|v\|_{1}$. And the norm $\|v\|_{\mathfrak{B}}$ is given by the sum of two graph norms corresponding to two maximal domains.

By definition, $C_{c}^{\infty}(X \backslash Y)$ is dense in $\mathfrak{B}$ and $\mathfrak{W}$ is a reflexive space. Denote by $\mathfrak{B}^{*}$ its dual space.

We will look for a singular solution to 4.22 of the form $u=e+v$, where $v \in \mathfrak{B}, e$ is an approximate solution determined by condition

$$
A^{\prime} \mathcal{F}(x, A e)+C^{\prime} \mathcal{G}(x, B e) \in \mathfrak{B}^{*}
$$

Moreover, let us pick a subset $\Xi \subset \mathfrak{B}$ such that $\Xi \cap \operatorname{Ker} A=\{0\}$. Using Corollary 4.27, the graph norms of functions in $D\left(A_{\max }^{\gamma, p}\right)$ and in $D\left(A_{\max }^{\rho, 2}\right)$ are equivalent to the seminorms

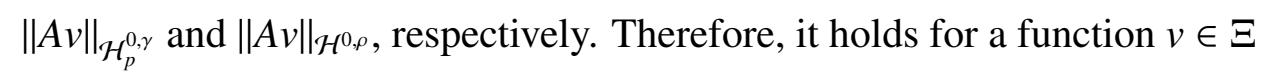

$$
\|v\|_{\mathfrak{B}} \sim\left(\int_{X}\|A v(x)\|_{F}^{2}\|A e(x)\|_{F}^{p-2} d \mu\right)^{\frac{1}{2}}+\left(\int_{X}\|A v(x)\|^{p} d \mu\right)^{\frac{1}{p}} .
$$


Furthermore, because of the condition $v_{0}+v_{1}<2 v$, we can assume that one of the mappings $B: \mathfrak{W} \rightarrow \mathcal{H}_{p}^{0, \gamma}\left(X ; G_{0}\right) \cap \mathcal{H}^{0, \rho}\left(X ; G_{0}\right)$ and $C: \mathfrak{B} \rightarrow \mathcal{H}_{p}^{0, \gamma}\left(X ; G_{1}\right) \cap \mathcal{H}^{0, \rho}\left(X ; G_{1}\right)$ is a compact operator. We can reduce (4.22) to an operator equation as follows:

Theorem 4.36. Let $p>2$. Suppose that $A^{\prime} A$ is an elliptic operator with respect to $\gamma, \gamma+\nu$, and at least one of the mappings $B: \mathfrak{W} \rightarrow \mathcal{H}_{p}^{0, \gamma}\left(X ; G_{0}\right) \cap \mathcal{H}^{0, \rho}\left(X ; G_{0}\right)$ and $C: \mathfrak{B} \rightarrow \mathcal{H}_{p}^{0, \gamma}\left(X ; G_{1}\right) \cap \mathcal{H}^{0, \rho}\left(X ; G_{1}\right)$ is a compact operator. Suppose further that the nonlinearity $\mathcal{F}$ satisfies the growth conditions (4.23), (4.24) and $\mathcal{G}: X \times G_{0} \rightarrow G_{1}^{\prime}$ satisfies

$$
\left\|\mathcal{G}\left(x, z_{1}\right)-\mathcal{G}\left(x, z_{2}\right)\right\|_{G_{1}^{\prime}} \leq C\left\|z_{1}-z_{2}\right\|_{G_{0}}\left(\left\|z_{1}\right\|_{G_{0}}^{p-2-\varepsilon}+\left\|z_{2}\right\|_{G_{0}}^{p-2-\varepsilon}\right)
$$

for all $\left(x, z_{i}\right) \in G_{0}, i=1,2$, here $0<\varepsilon<p-2$.

Then the equation (4.22) can be reduced to an operator equation, where the induced operator has property $\alpha$.

Proof. Let us define an operator $\widetilde{\mathbf{F}}: \mathfrak{W} \rightarrow \mathfrak{B}^{*}$ by

$$
\widetilde{\mathbf{F}} v=A^{\prime} \mathcal{F}(A(e+v))-A^{\prime} \mathcal{F}(A e) .
$$

The operator $\widetilde{\mathbf{F}}$ is well-defined, because for an arbitrary test function $w \in C_{c}^{\infty}(X \backslash Y)$ and $v \in \mathfrak{B}$, we have

$$
\begin{aligned}
|\langle\widetilde{\mathbf{F}} v, w\rangle|= & \left|\int_{X} h_{F}(\mathcal{F}(A(e+v))-\mathcal{F}(A e), A w) d \mu\right| \\
\leq & C \int_{X}\left(\|A e(x)\|_{F}^{p-2}+\|A(e+v)(x)\|_{F}^{p-2}\right)\|A v(x)\|_{F}\|A w(x)\|_{F} d \mu \text {, use (4.24) } \\
\leq & C \int_{X}\left(\|A e(x)\|_{F}^{p-2}+\|A v(x)\|_{F}^{p-2}\right)\|A v(x)\|_{F}\|A w(x)\|_{F} d \mu \text {, use 2.14) } \\
\leq & C\left[\left(\int_{X}\|A v(x)\|_{F}^{2}\|A e(x)\|_{F}^{p-2} d \mu\right)^{\frac{1}{2}}\left(\int_{X}\|A w(x)\|_{F}^{2}\|A e(x)\|_{F}^{p-2} d \mu\right)^{\frac{1}{2}}+\right. \\
& \left.+\left(\int_{X}\|A v(x)\|^{p} d \mu\right)^{1-\frac{1}{p}} \times\left(\int_{X}\|A w(x)\|^{p} d \mu\right)^{\frac{1}{p}}\right], \text { use Hölder inequality. }
\end{aligned}
$$

Therefore, by density argument, it holds for all $v, w \in \mathfrak{B}$ that

$$
|\langle\widetilde{\mathbf{F}} v, w\rangle| \leq C\left(\|v\|_{\mathfrak{B}}\right)\|w\|_{\mathfrak{B}}
$$

This inequality also implies that $\widetilde{\mathbf{F}}$ is a bounded operator, because

$$
\|\widetilde{\mathbf{F}} v\|_{\mathfrak{M}^{*}} \leq C\|v\|_{\mathfrak{M}}
$$


We verify the continuity of $\widetilde{\mathbf{F}}$ by similar proof. Indeed, given an arbitrary sequence $\left\{v_{n}\right\}$ which converges to $v$ in $\mathfrak{B}$, then it holds for arbitrary $w \in C_{c}^{\infty}(X \backslash Y)$ :

$$
\begin{aligned}
&\left|\left\langle\widetilde{\mathbf{F}} v_{n}-\widetilde{\mathbf{F}} v, w\right\rangle\right|=\left|\int_{X} h_{F}\left(\mathcal{F}\left(A\left(e+v_{n}\right)\right)-\mathcal{F}(A(e+v)), A w\right) d \mu\right| \\
& \leq C \int_{X}\left(\|A(e+v)(x)\|_{F}^{p-2}+\left\|A\left(v_{n}-v\right)(x)\right\|_{F}^{p-2}\right)\left\|A\left(v_{n}-v\right)(x)\right\|_{F}\|A w(x)\|_{F} d \mu \\
& \leq C \int_{X}\left(\|A e(x)\|_{F}^{p-2}+\left\|A\left(v_{n}-v\right)(x)\right\|_{F}^{p-2}\right)\left\|A\left(v_{n}-v\right)(x)\right\|_{F}\|A w(x)\|_{F} d \mu \\
& \leq C {\left[\left(\int_{X}\|A e(x)\|_{F}^{p-2}\left\|A\left(v_{n}-v\right)(x)\right\|_{F}^{2} d \mu\right)^{\frac{1}{2}}\left(\int_{X}\|A e(x)\|_{F}^{p-2}\|A w(x)\|_{F}^{2} d \mu\right)^{\frac{1}{2}}+\right.} \\
&+\left(\int_{X}\left\|A\left(v_{n}-v\right)(x)\right\|_{F}^{p} d \mu\right)^{1-\frac{1}{p}}\left(\int_{X}\|A w(x)\|_{F}^{p} d \mu\right)^{\frac{1}{p}}+ \\
&+\left.\left(\int_{X}\left\|A\left(v_{n}-v\right)(x)\right\|_{F}^{p} d \mu\right)^{1-\frac{2}{p}}\left(\int_{X}\|A w(x)\|_{F}^{p} d \mu\right)^{\frac{1}{p}}\left(\int_{X}\|A v(x)\|_{F}^{p} d \mu\right)^{\frac{1}{p}}\right]
\end{aligned}
$$

So the left-hand side $\left\langle\widetilde{\mathbf{F}} v_{n}-\widetilde{\mathbf{F}} v, w\right\rangle \rightarrow 0$ when $n \rightarrow \infty$ for all $w \in C_{c}^{\infty}(X \backslash Y)$. By density argument, it holds for all $w \in \mathfrak{X}$. So $\widetilde{\mathbf{F}}$ is continuous.

The second nonlinearity in 4.22 induces an operator $\widetilde{\mathbf{G}}: \mathfrak{B} \rightarrow \mathfrak{B}^{*}$ which is determined by the formula

$$
\widetilde{\mathbf{G}} v=C^{\prime} \mathcal{G}(x, B e+B v)-C^{\prime} \mathcal{G}(x, B e), v \in \mathfrak{B} .
$$

For a test function $w \in C_{c}^{\infty}(X \backslash Y)$, the action of this operator can be represented by the integral

$$
\langle\widetilde{\mathbf{G}} v, w\rangle=\int_{X} h_{E}(\mathcal{G}(x, B e+B v)-\mathcal{G}(x, B e), C w) d \mu .
$$

For such $w$, we can estimate

$$
\begin{aligned}
|\langle\widetilde{\mathbf{G}} v, w\rangle| & \leq \int_{X}|C w \| B v|\left(|B e+B v|^{p-2-\varepsilon}+|B e|^{p-2-\varepsilon}\right) d \mu \\
& \leq \int_{X}\|C w(x)\|_{G_{1}}\|B v(x)\|_{G_{0}}\left(\|B v(x)\|_{G_{0}}^{p-2-\varepsilon}+\|B e(x)\|_{G_{0}}^{p-2-\varepsilon}\right) d \mu \\
& \leq \int_{X}\left(\|C w(x)\|_{G_{1}}\|B v(x)\|_{G}\|B e(x)\|_{G}^{p-2-\varepsilon}+\|C w(x)\|_{G_{1}}\|B v(x)\|_{G_{0}}^{p-1-\varepsilon}\right) d \mu .
\end{aligned}
$$

Applying Hölder inequality to estimate each term, we obtain

$$
\int_{X}\|C w\|_{G_{1}}\|B v(x)\|_{G_{0}}^{p-1-\varepsilon} d \mu \leq C\|B v\|_{\mathcal{H}_{p-\varepsilon}^{0, p \gamma /(p-\varepsilon)}}^{p-1-\varepsilon}\|w\|_{\mathcal{H}_{p-\varepsilon}^{0, p \gamma /(p-\varepsilon)}} \leq C\|v\|_{\mathfrak{B}}^{p-1-\varepsilon}\|w\|_{\mathfrak{B}}
$$


and

$$
\begin{aligned}
\int_{X}\|C w(x)\|_{G_{1}}\|B v(x)\|_{G_{0}}\|B e(x)\|_{G}^{p-2-\varepsilon} d \mu \leq & \left(\int_{X}\|B v(x)\|_{G_{0}}^{2}\|B e(x)\|_{G}^{p-2-\varepsilon} d \mu\right)^{1 / 2} \times \\
& \times\left(\int_{X}\|C w(x)\|_{G_{1}}^{2}\|B e(x)\|_{G}^{p-2-\varepsilon} d \mu\right)^{1 / 2} \\
\leq & C\|B v\|_{\mathcal{H}^{0, \tilde{\gamma}}}\|C w\|_{\mathcal{H}^{0, \tilde{\gamma}}} \leq C\|v\|_{\mathfrak{B}}\|w\|_{\mathfrak{B}}
\end{aligned}
$$

where $\widetilde{\gamma}=\frac{(p-2-\varepsilon)\left(\lambda_{0}+v\right)}{2}+\frac{p \gamma}{2}$, and we have used the embeddings

$$
\begin{aligned}
& \mathcal{H}_{p}^{0, \gamma}(X ; E) \hookrightarrow \mathcal{H}_{p-\varepsilon}^{0, p \gamma /(p-\varepsilon)}(X ; E), \\
& \mathcal{H}^{0, \rho}\left(X ; G_{0}\right) \hookrightarrow \mathcal{H}^{0, \widetilde{\gamma}}\left(X ; G_{0}\right), \mathcal{H}^{0, \rho}\left(X ; G_{1}\right) \hookrightarrow \mathcal{H}^{0, \widetilde{\gamma}}\left(X ; G_{1}\right)
\end{aligned}
$$

We have shown that the operator $\widetilde{\mathbf{G}}$ is well-defined. Repeating the proof in the part above, we obtain also the continuity of $\widetilde{\mathbf{G}}$. Moreover, $\widetilde{\mathbf{G}}: \mathfrak{W} \rightarrow \mathfrak{B}^{*}$ is a compact operator under the assumption that one of the mappings $B: \mathfrak{B} \rightarrow \mathcal{H}_{p}^{0, \gamma}\left(X ; G_{0}\right) \cap \mathcal{H}^{0, \rho}\left(X ; G_{0}\right)$ and $C: \mathfrak{B} \rightarrow$ $\mathcal{H}_{p}^{0, \gamma}\left(X ; G_{1}\right) \cap \mathcal{H}^{0, \rho}\left(X ; G_{1}\right)$ is compact, see the proof of Lemma 4.31 .

Therefore, the operator $\widetilde{\mathbf{F}}+\widetilde{\mathbf{G}}$ satisfies property $\alpha$, provided that $\widetilde{\mathbf{F}}$ fulfills such property. However, this fact can be proved by repeating the proof in second-order case. What counts is the special structure of the nonlinearity $\mathcal{F}$.

Now we require a coercivity condition.

Theorem 4.37. Suppose all assumptions as in Theorem 4.36. Furthermore, let one of the following conditions hold for $\left(x, q_{1}\right),\left(x, q_{2}\right) \in F$ and $\left(x, z_{1}\right),\left(x, z_{2}\right) \in G_{0}$ :

a) If $B=C$ and $\mathcal{G}$ is a nondecreasing function.

b) $\left\|\mathcal{G}\left(x, z_{1}\right)-\mathcal{G}\left(x, z_{2}\right)\right\|_{G_{1}^{\prime}} \leq C\left\|z_{1}-z_{2}\right\|_{G_{0}}^{\sigma}\left(\left\|z_{1}\right\|_{G_{0}}^{p-2-\varepsilon}+\left\|z_{2}\right\|_{G_{0}}^{p-2-\varepsilon}\right), 0<\sigma<1$.

c) For $\left(x, \bar{z}_{1}\right),\left(x, \bar{z}_{2}\right) \in G_{1}$, it holds

$$
\left\langle\mathcal{F}\left(x, q_{1}\right)-\mathcal{F}\left(x, q_{2}\right), q_{1}-q_{2}\right\rangle+\left\langle\mathcal{G}\left(x, z_{1}\right)-\mathcal{G}\left(x, z_{2}\right), \bar{z}_{1}-\bar{z}_{2}\right\rangle \geq\left(\left|q_{1}\right|^{p-2}+\left|q_{2}\right|^{p-2}\right)\left|q_{1}-q_{2}\right|^{2}
$$

Then for any $f \in \Xi^{*}$ the equation (4.22) has a solution $u$ belonging to the affine space $e+\Xi$.

Proof. This theorem is proved by repeating verbatim the proof of Theorem 3.15 in the case a), Theorem 3.14 in the case b), and Theorem 3.16 in the case c). 


\section{Conclusion}

In my dissertation, I have answered the questions about existence of finite energy and singular solutions to general second-order and higher-order equations on manifolds with conic points. I have generalized results on existence of finite energy solutions in Euclidean spaces setting to the singular manifolds setting by adapting functional tools. The thesis contains the first attempt to use topological methods to prove existence of singular solutions. To achieve this goal, I have assumed existence of a formal solution with prescribed singular exponent and looked for remainder part in the intersection of two weighted Sobolev spaces, whose norms are controlled partly by the leading order of the formal solution. I have proved a Poincaré-type inequality and embedding theorems. Taking into account the fact that the formal solution satisfies the equation to infinite order, I have found a reduction of the original equation to an operator one, which is solvable by degree theory of generalized monotone mappings.

In this thesis, I dealt with nonlinearities satisfying special growth conditions which generalize the structure of the $p$-Laplacian in the case $p \geq 2$. Among the growth conditions is the coercivity assumption. My interest in the future will be further deploying the topological methods to prove existence for $p<2$ and when the coercivity assumption is relaxed. There are examples in the smooth case concerning finite energy solutions, as in example 2.34. However, there are no examples for singular solutions. The next goal of my research will be to extend the range of nonlinearity of the lower order terms involved and to prove existence results of equations on other kind of singular manifolds. 



\section{A Appendix}

\section{A.1 Degree of mappings of class $\alpha$}

The definition 2.25 actually is based on the approximation of the continuous mapping $f: \bar{\Omega} \rightarrow \mathbb{R}^{n}$ by a continuously differentiable mapping $g: \bar{\Omega} \rightarrow \mathbb{R}^{n}$ such that

$$
\max _{x \in \partial \Omega}|f(x)-g(x)|<\min _{x \in \partial \Omega}|f(x)|
$$

and at the points $x \in \Omega$ where $g(x)=0$, the Jacobian $\frac{D g(x)}{D x}$ is different from 0. By Sard's theorem, such approximation is valid. By definition,

$$
\operatorname{deg}(f, \bar{\Omega}, 0)=\operatorname{deg}(g, \bar{\Omega}, 0)=\sum_{x} \operatorname{sign} \frac{D g}{D x}(x)
$$

where $x \in \Omega$ satisfies $g(x)=0$.

The degree of generalized monotone mappings was introduced by Browder and Skrypnik independently in the late of the nineteen-sixties, which possesses all properties of the degree of finite-dimensional mappings mentioned in Section 2.3.2. We summarize here the construction by Skrypnik for mappings from a real separable reflexive Banach space to its dual. For the case of nonseparable space, we refer to Skrypnik's monograph [38].

Let $\left\{v_{n}\right\}_{n \in \mathbb{N}}$ be a complete system of the space $X$ and suppose that for every $n$, the elements $v_{1}, \ldots, v_{n}$ are linearly independent. Denote by $F_{n}$ the linear hull of the elements $v_{1}, \ldots, v_{n}$. Given a bounded open subset $D$ of $X$, let us denote $D_{n}:=D \cap F_{n}$, which is a finite dimensional space. We define finite-dimensional approximations $T_{n}$ of the mapping $T: \bar{D} \rightarrow X^{*}$ as follows:

$$
T_{n} u:=\sum_{i=1}^{n}\left\langle T u, v_{i}\right\rangle v_{i}, \text { for } u \in \bar{D}_{n} .
$$

Then $T_{n}$ is a mapping from $X$ to $F_{n}$.

Theorem A.1 ([38, Chapter 2, Theorem 2.1]). Let $T$ be an operator satisfying two conditions $a$ ), $b$ ) in Section 2.3.2. Then there exists a number $N$ such that for $n \geq N$ the following assertions are true: 
1) the equation $T_{n} u=0$ has no solution belonging to $\partial D_{n}$,

2) the degree $\operatorname{deg}\left(T_{n}, \bar{D}_{n}, 0\right)$ of the mapping $T_{n}$ with respect to $0 \in F_{n}$ is well-defined and independent of $n$.

Proof. We prove the first assertion by contradiction. Assume that there is a sequence $u_{k} \in \partial D_{n_{k}}$ such that $n_{k} \rightarrow \infty$ as $k \rightarrow \infty$ and $T_{n_{k}} u_{k}=0$. Because $X$ is reflexive, passing to a subsequence, we can consider that $u_{k} \rightarrow u_{0} \in X$. In addition, $T_{n_{k}} u_{k}=0$ implies that $T u_{k} \rightarrow 0$.

Taking any sequence $\omega_{k} \in F_{n_{k}}$ such that $\omega_{k} \rightarrow u_{0}$. Then

$$
\left\langle T u_{k}, u_{k}-u_{0}\right\rangle=\left\langle T u_{k}, \omega_{k}-u_{0}\right\rangle+\left\langle T u_{k}, u_{k}-\omega_{k}\right\rangle .
$$

Because $u_{k}-\omega_{k} \in F_{n_{k}}$, and $T_{n_{k}} u_{k}=0$, the second term vanishes. The first term also vanishes due to the boundedness of the operator $T$ and the strong convergence of $\omega_{k}$ to $u_{0}$. Therefore, $\lim _{k \rightarrow \infty}\left\langle T u_{k}, u_{k}-u_{0}\right\rangle=0$.

Since $T$ satisfies the condition $\alpha_{0}(\partial D)$, we obtain the strong convergence of $u_{k}$ (belonging to $\partial D$ ) to $u_{0}$. Therefore, $u_{0} \in \partial D$ and $T u_{0}=0$. This fact contradicts to the assumption b).

Once the first assertion is true, the degree $\operatorname{deg}\left(T_{n}, \bar{D}_{n}, 0\right)$ is well-defined.

We now introduce the mappings $\widetilde{T}_{n}: X \rightarrow F_{n}$ :

$$
\widetilde{T_{n}} u:=\sum_{i=1}^{n-1}\left\langle T u, v_{i}\right\rangle v_{i}+\left\langle h_{n}, u\right\rangle v_{n},
$$

where $h_{n} \in X^{*}$ satisfying the conditions $\left\langle h_{n}, v_{i}\right\rangle=0$ for $i<n$, and $\left\langle h_{n}, v_{n}\right\rangle=1$.

Lemma A.2 ([38, Chapter 2, Lemma 1.1]). [Leray-Schauder lemma] Let $f: \bar{\Omega} \rightarrow \mathbb{R}^{n}$ be a continuous mapping such that

$$
F_{n}\left(x_{1}, \ldots, x_{n}\right) \equiv x_{n},\left(x_{1}, \ldots, x_{n}\right) \in \Omega
$$

Suppose that $f(x) \neq 0$ for $x \in \partial \Omega$ and that the intersection $\Omega^{\prime}=\Omega \cap\left\{x: x_{n}=0\right\}$ is non empty. Then

$$
\operatorname{deg}(f, \bar{\Omega}, 0)=\operatorname{deg}\left(f^{\prime}, \overline{\Omega^{\prime}}, 0\right)
$$

where $f^{\prime}: \Omega^{\prime} \rightarrow \mathbb{R}^{n-1}$ defined by the equality

$$
f^{\prime}\left(x_{1}, \ldots, x_{n-1}\right)=\left(f_{1}\left(x_{1}, \ldots, x_{n-1}, 0\right), \ldots, f_{n-1}\left(x_{1}, \ldots, x_{n-1}, 0\right)\right) .
$$


By the Leray-Schauder lemma, we obtain that

$$
\operatorname{deg}\left(T_{n-1}, \bar{D}_{n-1}, 0\right)=\operatorname{deg}\left(\widetilde{T}_{n}, \bar{D}_{n}, 0\right)
$$

It remains to check that

$$
\operatorname{deg}\left(T_{n}, \bar{D}_{n}, 0\right)=\operatorname{deg}\left(\widetilde{T}_{n}, \bar{D}_{n}, 0\right)
$$

If it is true, then using homotopy invariance of finite-dimensional mappings, we achieve that

$$
\operatorname{deg}\left(T_{n-1}, \bar{D}_{n-1}, 0\right)=\operatorname{deg}\left(T_{n}, \bar{D}_{n}, 0\right)
$$

Indeed, we will prove that

$$
\left[t T_{n}+(1-t) \widetilde{T}_{n}\right] u \neq 0, \text { for } u \in \partial D_{n}, t \in[0,1]
$$

We prove this claim by contradiction. Assume that there exist sequences $u_{k} \in \partial D_{n_{k}}$ and $t_{k} \in[0,1], n_{k} \rightarrow \infty$ as $k \rightarrow \infty$ such that

$$
\left[t_{k} T_{n_{k}}+\left(1-t_{k}\right) \widetilde{T}_{n_{k}}\right] u_{k}=0
$$

This equality implies

$$
\begin{aligned}
& \left\langle T u_{k}, v_{i}\right\rangle=0 \text { for } i \leq n_{k}-1, \\
& t_{k}\left\langle T u_{k}, v_{n_{k}}\right\rangle+\left(1-t_{k}\right)\left\langle h_{n_{k}}, u_{k}\right\rangle=0 .
\end{aligned}
$$

Because of the first assertion and (A.1), A.2, we obtain that $T u_{k} \rightarrow 0$ and $0<t_{k}<1$ for $k$ large enough. We may assume that the sequence $u_{k}$ converges weakly to some element $\tilde{u}_{0} \in X$ and choose another sequence $\tilde{\omega}_{k} \in F_{n_{k}-1}$ which converges strongly to $\tilde{u}_{0}$. We have

$$
\left\langle T u_{k}, u_{k}-\tilde{u}_{0}\right\rangle=\left\langle T u_{k}, \tilde{\omega}_{k}-\tilde{u}_{0}\right\rangle+\left\langle T u_{k}, u_{k}-\tilde{\omega}_{k}\right\rangle
$$

The first term on the right-hand side tends to zero.

Since $\tilde{\omega}_{k} \in F_{n_{k}-1},\left\langle T u_{k}, \tilde{\omega}_{k}\right\rangle=0$. Using A.2 , we obtain

$$
\left\langle T u_{k}, u_{k}\right\rangle=-\frac{1-t_{k}}{t_{k}}\left\langle h_{n_{k}}, u_{k}\right\rangle^{2} .
$$

Therefore, $\lim _{k \rightarrow \infty}\left\langle T u_{k}, u_{k}-\tilde{u}_{0}\right\rangle=0$. By the condition $\alpha_{0}(\partial D)$, we deduce the strong convergence of $u_{k}$ to $\tilde{u}_{0} \in \partial D$. The identity (A.1) yields $T \tilde{u}_{0}=0$, which contradicts condition $b)$.

Now, the limit $\lim _{n \rightarrow \infty} \operatorname{deg}\left(T_{n}, \bar{D}_{n}, 0\right)$ exists and we denote it by $D\left\{v_{i}\right\}$. 
Theorem A.3 ([38, Chapter 2, Theorem 2.2]). Suppose that the conditions a), b) in Section 2.3.2 hold. Then the limit

$$
D\left\{v_{i}\right\}=\lim _{n \rightarrow \infty} \operatorname{deg}\left(T_{n}, \bar{D}_{n}, 0\right)
$$

does not depend on the choice of the sequence $\left\{v_{i}\right\}$.

Proof. We will show that $D\left\{v_{i}\right\}=D\left\{v_{i}^{\prime}\right\}$ for any other sequence $\left\{v_{i}^{\prime}\right\}$ with the same properties as the sequence $\left\{v_{i}\right\}$. We use the notations $A_{n}^{\prime}, D_{n}^{\prime}$ corresponding to $\left\{v_{n}\right\}$.

Assume that for each $n$, the system $v_{1}, \ldots, v_{n}, v_{1}^{\prime}, \ldots, v_{n}^{\prime}$ is linearly independent. Otherwise, we can always construct a third system $\widetilde{v_{1}}, \ldots, \widetilde{v_{n}}$ such that the systems $v_{1}, \ldots, v_{n}, \widetilde{v_{1}}, \ldots, \widetilde{v_{n}}$ and $v_{1}^{\prime}, \ldots, v_{n}^{\prime}, \widetilde{v_{1}}, \ldots, \widetilde{v_{n}}$ are linearly independent. And we will use $D\left\{\widetilde{v_{i}}\right\}$ as the intermediate value to compare $D\left\{v_{i}\right\}$ and $D\left\{v_{i}^{\prime}\right\}$.

Let $L_{2 n}$ be the linear space spanned by the elements $v_{1}, \ldots, v_{n}, v_{1}^{\prime}, \ldots, v_{n}^{\prime}$ and define the following finite-dimensional mappings:

$$
T_{2 n, t} u=\sum_{i=1}^{n}\left\{\left\langle T u, v_{i}\right\rangle v_{i}+\left[t\left\langle T u, v_{i}^{\prime}\right\rangle+(1-t)\left\langle f_{i}^{(n)}, u\right\rangle\right] v_{i}^{\prime}\right\},
$$

where $u \in \bar{D} \cap L_{2 n}, t \in[0,1]$ and $f_{i}^{(n)} \in X^{*}$ such that

$$
\left\langle f_{i}^{(n)}, v_{j}\right\rangle=0,\left\langle f_{i}^{(n)}, v_{j}^{\prime}\right\rangle=\delta_{i j}, i, j=1, \ldots, n
$$

By theorem A.1. we need to show $\operatorname{deg}\left(T_{n}, \bar{D}_{n}, 0\right)=\operatorname{deg}\left(T_{n}^{\prime},{\overline{D^{\prime}}}_{n}, 0\right)$ for $n$ large enough.

It suffices to verify that, for $n$ large,

$$
T_{2 n, t} u \neq 0 \text { for } u \in \partial\left(L_{2 n} \cap \bar{D}\right), t \in[0,1]
$$

If it is true, by using Leray-Schauder lemma, we have

$$
\operatorname{deg}\left(T_{2 n, 0}, L_{2 n} \cap \bar{D}, 0\right)=\operatorname{deg}\left(T_{n}, \bar{D}_{n}, 0\right) .
$$

On the other hand, due to homotopy invariant property, we have

$$
\operatorname{deg}\left(T_{2 n, 0}, L_{2 n} \cap \bar{D}, 0\right)=\operatorname{deg}\left(T_{2 n, 1}, L_{2 n} \cap \bar{D}, 0\right)
$$

We notice that $T_{2 n, 1}$ and $L_{2 n}$ are defined symmetrically in $v_{i}$ and $v_{i}^{\prime}$, therefore, we conclude that

$$
\operatorname{deg}\left(T_{n}, \bar{D}_{n}, 0\right)=\operatorname{deg}\left(T_{n}^{\prime},{\overline{D^{\prime}}}_{n}, 0\right)=\operatorname{deg}\left(T_{2 n, 1}, L_{2 n} \cap \bar{D}, 0\right)
$$


Assume that the assertion above is not true. Then there are sequences $\bar{u}_{k} \in \partial\left(L_{2 n_{k}} \cap \bar{D}\right)$ and $\bar{t}_{k} \in[0,1]$ such that

$$
T_{2 n_{k}, \bar{t}_{k}} \bar{u}_{k}=0, n_{k} \rightarrow \infty \text { as } k \rightarrow \infty .
$$

This yields that

$$
\begin{aligned}
& \left\langle T \bar{u}_{k}, v_{i}\right\rangle=0, i=1, \ldots, n_{k}, \\
& \bar{t}_{k}\left\langle A \bar{u}_{k}, v_{i}^{\prime}\right\rangle+\left(1-\bar{t}_{k}\right)\left\langle f_{i}^{\left(n_{k}\right)}, \bar{u}_{k}\right\rangle=0 .
\end{aligned}
$$

By theorem A.1, $0<\bar{t}_{k}<1$ for $k$ large enough. Moreover, we know $\bar{u}_{k} \rightarrow \bar{u}_{0}, \bar{t}_{k} \rightarrow \bar{t}_{0}$, and $T \bar{u}_{k} \rightarrow 0$ as $k \rightarrow \infty$. We proceed as in the proof above. Choose a sequence $\bar{\omega}_{k} \in F_{n_{k}}$ which converges strongly to $\bar{u}_{0}$. Using the formula

$$
\bar{u}_{k}=\sum_{i=1}^{n_{k}}\left(c_{i}^{(k)} v_{i}+\bar{c}_{i}^{(k)} v_{i}^{\prime}\right), \bar{c}_{i}^{(k)}=\left\langle f_{i}^{\left(n_{k}\right)}, \bar{u}_{k}\right\rangle,
$$

from the identities (A.3), A.4 , we obtain

$$
\begin{aligned}
\left\langle T \bar{u}_{k}, \bar{u}_{k}-\bar{u}_{0}\right\rangle & =\left\langle T \bar{u}_{k}, \bar{\omega}_{k}-\bar{u}_{0}\right\rangle+\sum_{i=1}^{n_{k}}\left\langle T \bar{u}_{k}, v_{i}^{\prime}\right\rangle\left\langle f_{i}^{\left(n_{k}\right)}, \bar{u}_{k}\right\rangle \\
& =\left\langle T \bar{u}_{k}, \bar{\omega}_{k}-\bar{u}_{0}\right\rangle-\frac{1-\bar{t}_{k}}{\bar{t}_{k}} \sum_{i=1}^{n_{k}}\left\langle f_{i}^{\left(n_{k}\right)}, \bar{u}_{k}\right\rangle^{2} .
\end{aligned}
$$

Therefore, $\lim \sup _{k \rightarrow \infty}\left\langle T \bar{u}_{k}, \bar{u}_{k}-\bar{u}_{0}\right\rangle \leq 0$. That $T$ satisfies the condition $\alpha_{0}(\partial D)$ implies the strong convergence of $\bar{u}_{k}$ to $\bar{u}_{0} \in \partial D$. The demicontinuity of $A$ and the fact that $A u_{k} \rightarrow 0$ as $k \rightarrow \infty$ imply that $T \bar{u}_{0}=0$, which contradicts the assumption b). This completes the proof.

Definition A.4 ([38, Chapter 2, Definition 2.4]). For an operator $T$ satisfying conditions a), b) in Section 2.3.2, its degree $\operatorname{Deg}(T, \bar{D}, 0)$ on the set $\bar{D}$ with respect to the point $0 \in X^{*}$ is the number

$$
\operatorname{Deg}(T, \bar{D}, 0):=\lim _{n \rightarrow \infty} \operatorname{deg}\left(T_{n}, \bar{D}_{n}, 0\right)
$$

\section{A.2 Closed extensions of symmetric operators}

We summarize here descriptions of the minimal and maximal domains of an elliptic cone differential operator $A \in \operatorname{Diff}_{c}^{v}(X)$ considered as an unbounded operator $C_{c}^{\infty}(X \backslash Y) \hookrightarrow$ $\mathcal{H}_{p}^{0, \gamma}(X) \rightarrow \mathcal{H}_{p}^{0, \gamma}(X)$. Assume that $A$ is written as

$$
A=t^{-v} \sum_{j=0}^{v} a_{j}(t)\left(-t \partial_{t}\right)^{j}, a_{j} \in C^{\infty}\left([0,1), \operatorname{Diff}^{v-j}(Y)\right) .
$$


By definition, considering $A$ as a mapping on $\mathcal{H}_{p}^{0, \gamma}(X)$, the minimal domain is the closure of $C_{c}^{\infty}(X \backslash Y)$ with respect to the graph norm

$$
\|u\|=\|u\|_{\mathcal{H}_{p}^{0, \gamma}}+\|A u\|_{\mathcal{H}_{p}^{0, \gamma}},
$$

and the maximal domain is the set

$$
\left\{u \in \mathcal{H}_{p}^{0, \gamma}(X) \mid A u \in \mathcal{H}_{p}^{0, \gamma}(X)\right\}
$$

We state without proof the following well-known results in [32].

Proposition A.5 ([32, Proposition 2.3]). The minimal domain is

$$
\begin{aligned}
D\left(A_{\min }\right) & =D\left(A_{\max }\right) \cap_{\varepsilon>0} \mathcal{H}_{p}^{\nu, v+\gamma-\varepsilon}(X) \\
& =\left\{u \in \cap_{\varepsilon>0} \mathcal{H}_{p}^{v, v+\gamma-\varepsilon}(X) \mid t^{-v} \sum_{j=0}^{v} a_{j}(0)\left(-t \partial_{t}\right)^{j}(\omega u) \in \mathcal{H}_{p}^{0, \gamma}(X)\right\} .
\end{aligned}
$$

In particular,

$$
\mathcal{H}_{p}^{\nu, v+\gamma}(X) \hookrightarrow D\left(A_{\min }\right) \hookrightarrow \mathcal{H}_{p}^{\nu, v+\gamma-\varepsilon}(X), \forall \varepsilon>0 .
$$

We have $D\left(A_{\min }\right)=\mathcal{H}_{p}^{v, v+\gamma}(X)$ if and only if the principal conormal symbol $\sigma_{M}^{v}(A)(z)$ is invertible for all $z$ with $\mathfrak{R} z=\frac{N+1}{p}-\gamma-v$.

When $A$ is an elliptic operator, the minimal domain has finite codimension in the maximal domain. This complementary space can be described by means of singular functions, whose singular exponents are just poles of the inverse of the principal conormal symbol lying in the strip

$$
\frac{N+1}{p}-\gamma-v \leq \mathfrak{R} p_{j}<\frac{N+1}{p}-\gamma .
$$

Indeed, because of ellipticity of $A$, its principal conormal symbol $\sigma_{M}^{v}(A)(z)$ is meromorphically invertible and its inverse can be written as the sum of a meromorphic Mellin symbol and a holomorphic one. Let us define recursively

$$
g_{0}:=\left(\sigma_{M}^{v}(A)(z)\right)^{-1}
$$

and

$$
g_{l}:=-\left(T^{-l} g_{0}\right) \sum_{j=0}^{l-1}\left(T^{-j} \sigma_{M}^{l-j}(A)(z)\right) g_{j}, l=1, \ldots, v-1 .
$$

Moreover, choose an $\varepsilon>0$ so small that every pole $p$ of one of the symbols $g_{0}, g_{1}, \ldots, g_{v-1}$ either lies on one of the lines $\mathfrak{R} z=\frac{N+1}{p}-\gamma-v+k$ or lies far from these lines at a distance larger than $\varepsilon$. Denote $\gamma_{p}=\gamma+\frac{N+1}{p}-\frac{N+1}{2}$. 
Definition A.6. Let $g_{0}, g_{1}, \ldots, g_{v-1}$ and $\varepsilon>0$ as above. Set

$$
\mathcal{E}=\mathcal{E}_{A}^{\gamma}=\operatorname{im} G_{0}+\operatorname{im} G_{1}+\ldots+\operatorname{im} G_{v-1}
$$

where the operators $G_{k}=\sum_{l=0}^{k} G_{k l}: C_{c}^{\infty}((0,1) \times Y) \rightarrow \mathcal{H}_{p}^{\infty, \gamma+\varepsilon}(X)$ are defined by

$$
G_{0}=G_{00}=\omega\left(\mathrm{op}_{M}^{\gamma_{p}+v+\varepsilon-1-\frac{N}{2}}\left(g_{0}\right)-\mathrm{op}_{M}^{\gamma_{p}+v+\varepsilon-\frac{N}{2}}\left(g_{0}\right)\right),
$$

and if $1 \leq k \leq v-1,0 \leq l \leq k$

$$
G_{k l}=\omega t^{l}\left(\mathrm{op}_{M}^{\gamma_{p}+\nu+\varepsilon-k-1-\frac{N}{2}}\left(g_{0}\right)-\mathrm{op}_{M}^{\gamma_{p}+\nu+\varepsilon-k-\frac{N}{2}}\left(g_{0}\right)\right),
$$

The space $\mathcal{E}$ is a finite-dimensional subspace of $C^{\infty, \gamma+\varepsilon}(X)$ consisting of functions of the form

$$
u(t, y)=\omega(t) \sum_{j=0}^{N} \sum_{k=0}^{m_{j}} \psi_{j k}(y) t^{-p_{j}} \log ^{k} t
$$

where $\psi_{j k}(y) \in C^{\infty}(Y)$ and

$$
\frac{N+1}{p}-\gamma-v \leq \mathfrak{R} p_{j}<\frac{N+1}{p}-\gamma
$$

Proposition A.7 ([32, Theorem 2.8]). The domain of the maximal extension $A(\max )$ is

$$
D\left(A_{\max }\right)=D\left(A_{\min }\right)+\mathcal{E},
$$

with $\mathcal{E}$ defined in $(\mathrm{A} .5$. The space $\mathcal{E}$ does not depend on $1<p<\infty$. Moreover,

$$
D\left(A_{\min }\right) \cap \mathcal{E} \subset \operatorname{im} \omega\left(\mathrm{op}_{M}^{\gamma_{p}+\nu-\varepsilon-\frac{N}{2}}\left(\sigma_{M}^{\nu}(A)^{-1}\right)-\mathrm{op}_{M}^{\gamma_{p}+\nu+\varepsilon-\frac{N}{2}}\left(\sigma_{M}^{v}(A)^{-1}\right)\right) .
$$

The sum in A.6 is direct particularly when $\sigma_{M}^{v}(A)^{-1}$ has no poles on the weight line $\mathfrak{R} z=\frac{N+1}{p}-\gamma-v$.

Consequently, any closed extension $\underline{\mathcal{A}}$ in $\mathcal{H}_{p}^{0, \gamma}(X)$ is given by the action of $A$ on a domain

$$
D(\underline{\mathcal{A}})=D\left(A_{\min }\right)+\underline{\mathcal{E}},
$$

where $\underline{\mathcal{E}}$ is a subspace of $\mathcal{E}$. 



\section{Bibliography}

[1] M. Baranowski. Totally characteristic pseudo-differential operators in BesovLizorkin-Triebel spaces. Ann. Global Anal. Geom., 7(1):3-27, 1989.

[2] M. F. Betta, A. Mercaldo, F. Murat, and M. M. Porzio. Existence of renormalized solutions to nonlinear elliptic equations with a lower-order term and right-hand side a measure. J. Math. Pures Appl. (9), 82(1):90-124, 2003. Corrected reprint of J. Math. Pures Appl. (9) 81 (2002), no. 6, 533-566 [ MR1912411 (2003e:35075)].

[3] M. Borsuk and V. Kondratiev. Elliptic boundary value problems of second order in piecewise smooth domains, volume 69 of North-Holland Mathematical Library. Elsevier Science B.V., Amsterdam, 2006.

[4] F. E. Browder. Nonlinear elliptic boundary value problems. Bull. Amer. Math. Soc., 69:862-874, 1963.

[5] H. Chen, X. Liu, and Y. Wei. Existence theorem for a class of semilinear totally characteristic elliptic equations with critical cone Sobolev exponents. Ann. Global Anal. Geom., 39(1):27-43, 2011.

[6] O. Costin. Asymptotics and Borel summability, volume 141 of Chapman $\mathcal{E}$ Hall/CRC Monographs and Surveys in Pure and Applied Mathematics. CRC Press, Boca Raton, FL, 2009.

[7] G. Dal Maso, F. Murat, L. Orsina, and A. Prignet. Renormalized solutions of elliptic equations with general measure data. Ann. Scuola Norm. Sup. Pisa Cl. Sci. (4), 28(4):741-808, 1999.

[8] J. I. Díaz. Nonlinear partial differential equations and free boundaries. Vol. I, volume 106 of Research Notes in Mathematics. Pitman (Advanced Publishing Program), Boston, MA, 1985. Elliptic equations. 
[9] P. Drábek, A. Kufner, and F. Nicolosi. Quasilinear elliptic equations with degenerations and singularities, volume 5 of de Gruyter Series in Nonlinear Analysis and Applications. Walter de Gruyter \& Co., Berlin, 1997.

[10] P. Drábek and J. Milota. Methods of nonlinear analysis. Birkhäuser Advanced Texts: Basler Lehrbücher. [Birkhäuser Advanced Texts: Basel Textbooks]. Birkhäuser Verlag, Basel, 2007. Applications to differential equations.

[11] Y. V. Egorov and B.-W. Schulze. Pseudo-differential operators, singularities, applications, volume 93 of Operator Theory: Advances and Applications. Birkhäuser Verlag, Basel, 1997.

[12] A. Friedman and L. Véron. Singular solutions of some quasilinear elliptic equations. Arch. Rational Mech. Anal., 96(4):359-387, 1986.

[13] J. B. Gil and G. A. Mendoza. Adjoints of elliptic cone operators. Amer. J. Math., 125(2):357-408, 2003.

[14] D. Gilbarg and N. S. Trudinger. Elliptic partial differential equations of second order. Classics in Mathematics. Springer-Verlag, Berlin, 2001. Reprint of the 1998 edition.

[15] P. Grisvard. Elliptic problems in nonsmooth domains, volume 24 of Monographs and Studies in Mathematics. Pitman (Advanced Publishing Program), Boston, MA, 1985.

[16] P. Grisvard. Singularities in boundary value problems, volume 22 of Recherches en Mathématiques Appliquées [Research in Applied Mathematics]. Masson, Paris, 1992.

[17] J. Heinonen, T. Kilpeläinen, and O. Martio. Nonlinear potential theory of degenerate elliptic equations. Oxford Mathematical Monographs. The Clarendon Press Oxford University Press, New York, 1993. Oxford Science Publications.

[18] P. Juutinen, P. Lindqvist, and J. J. Manfredi. On the equivalence of viscosity solutions and weak solutions for a quasi-linear equation. SIAM J. Math. Anal., 33(3):699-717 (electronic), 2001. 
[19] D. Kapanadze, B.-W. Schulze, and I. Witt. Coordinate invariance of the cone algebra with asymptotics. In Parabolicity, Volterra calculus, and conical singularities, volume 138 of Oper. Theory Adv. Appl., pages 307-358. Birkhäuser, Basel, 2002.

[20] S. Kichenassamy. Fuchsian reduction. Progress in Nonlinear Differential Equations and their Applications, 71. Birkhäuser Boston Inc., Boston, MA, 2007. Applications to geometry, cosmology, and mathematical physics.

[21] S. Kichenassamy and L. Véron. Singular solutions of the $p$-Laplace equation. Math. Ann., 275(4):599-615, 1986.

[22] A. Kufner, O. John, and S. Fučík. Function spaces. Noordhoff International Publishing, Leyden, 1977. Monographs and Textbooks on Mechanics of Solids and Fluids; Mechanics: Analysis.

[23] O. A. Ladyzhenskaya and N. N. Ural'tseva. Linear and quasilinear elliptic equations. Translated from the Russian by Scripta Technica, Inc. Translation editor: Leon Ehrenpreis. Academic Press, New York, 1968.

[24] J. Leray and J.-L. Lions. Quelques résulatats de Višik sur les problèmes elliptiques nonlinéaires par les méthodes de Minty-Browder. Bull. Soc. Math. France, 93:97$107,1965$.

[25] J. Leray and J. Schauder. Topologie et équations fonctionnelles. Ann. Sci. École Norm. Sup. (3), 51:45-78, 1934.

[26] M. Lesch. Operators of Fuchs type, conical singularities, and asymptotic methods, volume 136 of Teubner-Texte zur Mathematik [Teubner Texts in Mathematics]. B. G. Teubner Verlagsgesellschaft mbH, Stuttgart, 1997.

[27] X. Liu and I. Witt. Asymptotic expansions for bounded solutions to semilinear Fuchsian equations. Doc. Math., 9:207-250 (electronic), 2004.

[28] R. Mazzeo and F. Pacard. A construction of singular solutions for a semilinear elliptic equation using asymptotic analysis. J. Differential Geom., 44(2):331-370, 1996.

[29] B. Opic and A. Kufner. Hardy-type inequalities, volume 219 of Pitman Research Notes in Mathematics Series. Longman Scientific \& Technical, Harlow, 1990. 
[30] J. Peetre. Another approach to elliptic boundary problems. Comm. Pure Appl. Math., 14:711-731, 1961.

[31] E. Schrohe and J. Seiler. Ellipticity and invertibility in the cone algebra on $L_{p^{-}}$ Sobolev spaces. Integral Equations Operator Theory, 41(1):93-114, 2001.

[32] E. Schrohe and J. Seiler. The resolvent of closed extensions of cone differential operators. Canad. J. Math., 57(4):771-811, 2005.

[33] B.-W. Schulze. Boundary value problems and singular pseudo-differential operators. Pure and Applied Mathematics (New York). John Wiley \& Sons Ltd., Chichester, 1998.

[34] J. Seiler. The cone algebra and a kernel characterization of Green operators. In Approaches to singular analysis (Berlin, 1999), volume 125 of Oper. Theory Adv. Appl., pages 1-29. Birkhäuser, Basel, 2001.

[35] J. Serrin. Local behavior of solutions of quasi-linear equations. Acta Math., 111:247-302, 1964.

[36] J. Serrin. Isolated singularities of solutions of quasi-linear equations. Acta Math., 113:219-240, 1965.

[37] I. V. Skrypnik. Nonlinear elliptic boundary value problems, volume 91 of TeubnerTexte zur Mathematik [Teubner Texts in Mathematics]. BSB B. G. Teubner Verlagsgesellschaft, Leipzig, 1986. With German, French and Russian summaries.

[38] I. V. Skrypnik. Methods for analysis of nonlinear elliptic boundary value problems, volume 139 of Translations of Mathematical Monographs. American Mathematical Society, Providence, RI, 1994. Translated from the 1990 Russian original by Dan D. Pascali.

[39] M. Struwe. Variational methods. Springer-Verlag, Berlin, 1990. Applications to nonlinear partial differential equations and Hamiltonian systems.

[40] P. Tolksdorf. On the Dirichlet problem for quasilinear equations in domains with conical boundary points. Comm. Partial Differential Equations, 8(7):773-817, 1983. 
[41] L. Véron. Singularities of solutions of second order quasilinear equations, volume 353 of Pitman Research Notes in Mathematics Series. Longman, Harlow, 1996.

[42] I. Witt. Asymptotic algebras. Sūrikaisekikenkyūsho Kōkyūroku, (1211):21-33, 2001. Asymptotic analysis and microlocal analysis of PDE (Japanese) (Kyoto, 2000).

[43] I. Witt. Local asymptotic types. Manuscripta Math., 115(1):1-17, 2004.

[44] I. Witt. Green's formulas for cone differential operators. Trans. Amer. Math. Soc., 359(12):5669-5696, 2007.

[45] E. Zeidler. Nonlinear functional analysis and its applications. II/B. Springer-Verlag, New York, 1990. Nonlinear monotone operators, Translated from the German by the author and Leo F. Boron. 